\title{
CANONICAL SUBGROUPS OVER HILBERT MODULAR VARIETIES
}

\author{
EYAL Z. GOREN \& PAYMAN L KASSAEI
}

\begin{abstract}
We obtain new results on the geometry of Hilbert modular varieties in positive characteristic and morphisms between them. Using these results and methods of rigid geometry, we develop a theory of canonical subgroups for abelian varieties with real multiplication.
\end{abstract}

\section{INTRODUCTION}

The theory of canonical subgroups was developed by Katz [28, building on work of Lubin on canonical subgroups of formal groups of dimension one. Katz's motivation was to show that the $U_{p}$ operator on the space of $p$-adic elliptic modular forms preserves the subspace of overconvergent modular forms.

The kernel of multiplication by $p$ in the formal group of an elliptic curve of ordinary reduction over a $p$-adic base has a distinguished subgroup of order $p$, which reduces to the Kernel of Frobenius modulo $p$ and is called the canonical subgroup. The $U_{p}$ operator can be defined by a moduli-theoretic formula involving the canonical subgroup. Extending this Hecke operator to overconvergent modular forms directly involves extending the notion of canonical subgroups from elliptic curves of ordinary reduction to those of a "slight" supersingular reduction (quantified by an appropriate measure of supersingularity involving the Hasse invariant). However, Katz-Lubin proved much more: they provided optimal bounds on the measure of supersingularity for the existence of canonical subgroups, and proved that the canonical subgroup again reduces to the kernel of Frobenius, albeit only modulo a divisor of $p$ determined by a measure of supersingularity of $E$.

The power of canonical subgroups and their properties became apparent, for example, in the work of Buzzard-Taylor on the Artin conjecture [7, 6], where modularity of certain Galois represenations was proved by analytic continuation of overconvergent modular forms, and in the work of Kassaei on classicality of overconvergent modular forms in [26, 27, where analytic continuation of overconvergent modular forms was used to provide a method for proving Coleman's classicality theorem [10] for more general Shimura varieties; in both examples, canonical subgroups and their properties were used in the process of analytic continuation.

The story of $p$-adic modular forms began when Serre introduced them in 33 as $p$-adic limits of $q$-expansions of classical modular forms. The theory was motivated by two issues. The $p$-adic interpolation of constant terms of certain Eisenstein series, in particular special values of abelian $L$-functions, and the construction of $p$-adic analytic families of modular forms with connections to Galois representations and Iwasawa theory in mind. Serre's point of view can be generalized to the Hilbert modular case. This has been done by Andreatta-Goren 4, but already previously some aspects of the theory were generalized by Deligne-Ribet and the applications to constant terms

1991 Mathematics Subject Classification. Primary [11F85, 11F41]; Secondary [11G18, 14G35, 14G22]. 
of Eisenstein series were harvested [12]. Dwork studied p-adic modular functions with "growth condition" and showed that the $U_{p}$ operator is completely continuous on the space of these functions. Almost at the same time of Serre's work, Katz [28] interpreted Serre's p-adic modular forms as sections of suitable line bundles over the ordinary locus of the corresponding modular curve. Katz incorporated Dwork's notion of growth condition into this geometric construction by considering sections of the same line bundles over larger regions of the $p$-adic analytic space of the modular curve, thus giving birth to the notion of overconvergent modular forms.

As we mentioned above, the $p$-torsion in the formal group of an elliptic curve of ordinary reduction over a $p$-adic base provides a lifting of the Kernel of Frobenius modulo $p$. This is not hard to see using some fundamental facts about étale formal groups over a $p$-adic base. When the elliptic curve has supersingular reduction, the $p$-torsion of the formal group grows to a subgroup of rank $p^{2}$, no longer providing a canonical subgroup. Using Newton polygons, Katz-Lubin showed that for an elliptic curve of "not-too-supersingular" reduction there are $p-1$ non-zero elements in the $p$-torsion of the formal group which are of (equal and) closer distance to the origin than the remaining non-zero elements; along with zero, they from a distinguished subgroup of rank $p$ called the canonical subgroup of the elliptic curve. Katz showed that this construction works in families and consequently proved that the Hecke operator $U_{p}$ preserves the space of overconvergent modular forms. This completely continuous operator has been essential to the development of the theory of overconvergent modular forms (especially through the pioneering works of Hida and Coleman).

After Katz and Lubin the first major advancement in the study of the canonical subgroup was made by Abbes and Mokrane in [1]. Since then, many authors have studied the canonical subgroup in various settings and with different approaches. We mention the works [11, [16], [29], 35], as well as yet unpublished results by K. Buzzard, E. Nevens and J. Rabinoff. Broadly speaking, the traditional approach to the canonical subgroup problem proceeds through a careful examination of subgroup schemes of either abelian varieties, or $p$-divisible groups, and, again broadly speaking, much of the complications arise from the fact that formal groups in several variables are hard to describe and one lacks a theory of Newton polygons for power-series in several variables. These deficiencies, as well as Raynaud's interpretation of rigid spaces in the language of formal schemes, prompted us to investigate another approach to canonical subgroups, where geometry plays a more prominent role. In [20] we tested our ideas in the case of curves and showed that one can develop the theory of canonical subgroup for "all" Shimura curves at once, getting results that are, in a sense, more precise than previously known. As special cases, we recovered results of Katz-Lubin (modular curves) and 25] (unitary Shimura curves). In our setting, one considers a morphism $\pi: Y \rightarrow X$, where $X, Y$, are any "nice" curves over a dvr $R$, finite over $\mathbb{Z}_{p}$, such that the reductions of $\pi, X$, and $Y$, modulo the maximal ideal satisfy certain simple geometric properties known to be present in the case of Shimura curves. Canonical subgroups and their finer properties are then studied by constructing a section to the rigid analytic fibre of $\pi$ and studying its properties. This theory is used in [27] to prove classicality results for overconvergent modular forms simultaneously for "all" Shimura curves.

The general canonical subgroup problem can be formulated as follows. We restrict to Shimura varieties of PEL type, although one should be able to extend it to Shimura varieties of Hodge type, for example. The typical context is that one is given a moduli problem of PEL type and a 
fine moduli space $X$ representing it over $\operatorname{Spec}\left(\mathcal{O}_{K}\left[S^{-1}\right]\right)$ - a localization of the ring of integers of some number field $K$. If $p$ is a rational prime, such that the reductive group corresponding to the moduli problem is unramified at $p$ and the level structure is prime to $p, X$ usually has a smooth model over $R$, the completion of $\mathcal{O}_{K}\left[S^{-1}\right]$ at a prime $\mathfrak{p}$ above $p$. One then adds a suitable level structure at $p$ to the moduli problem represented by $X$ to obtain a fine moduli scheme $Y / \operatorname{Spec}(R)$ and a natural forgetful morphism $\pi: Y \rightarrow X$. Under minimal conditions, the reduction $\bar{X}$ of $X$ modulo $\mathfrak{m}_{R}$ has a generalized ordinary locus $\bar{X}^{\text {ord }}$, which is open and dense in $\bar{X}$, and a section $s: \bar{X}^{\text {ord }} \rightarrow \bar{Y}^{\text {ord }}$ to $\pi$. One certainly expects to be able to lift $s: \bar{X}^{\text {ord }} \rightarrow \bar{Y}^{\text {ord }}$ to a section $s^{\circ}: \mathfrak{X}^{\circ} \rightarrow \mathfrak{Y}^{\circ}$, where $\mathfrak{X}^{\circ}$ is the (admissible open) set in the rigid analytic space associated to $X$ consisting of the points that specialize to $\bar{X}$; similarly for $\mathfrak{Y}^{\circ}$. The canonical subgroup problem is to find an explicitly described admissible open set $\mathcal{U} \supseteq \mathfrak{X}^{\circ}$ to which one can extend $s^{\circ}$ (if one chooses $\mathcal{U}$ appropriately the extension is unique) and characterize its image.

The completed local rings $\widehat{\mathcal{O}}_{X, \bar{P}}, \widehat{\mathcal{O}}_{Y, \bar{Q}}$ at closed points $\bar{P}, \bar{Q}$ of the special fibers $\bar{X}, \bar{Y}$ afford an interpretation in terms of pro-representing a moduli problem on a category of certain local artinian rings. Using the theory of local models, one expects to be able to to write down models for these rings that are the completed local rings of suitable points on certain generalized Grassmann varieties.

Assume that $\pi(\bar{Q})=\bar{P}$. According to our approach, it is the map $\pi^{*}: \widehat{\mathcal{O}}_{X, \bar{P}} \rightarrow \widehat{\mathcal{O}}_{Y, \bar{Q}}$ that "holds all the secrets" concerning the canonical subgroup. In this paper we show that this is the case for Hilbert modular varieties. The information we can find on $\pi^{*}$ uses heavily the moduli description, but once obtained, the specific nature of $X, Y$, as moduli schemes plays no role anymore.

We find it remarkable that not only does this suffice for the construction of the canonical subgroup, in fact the results we obtain improve significantly on what is available in the literature as a consequence of work by others. For example, we are able to prove the existence of canonical subgroups on domains described by valuations of as many parameters as the dimension of $X$, whereas in the literature these constructions are almost always carried out on coarser domains defined by the valuation of one variable (the Hodge degree). Also our results improve significantly on the bounds for these variables; in fact these bounds can be shown to be optimal in a sense explained in Corollary 6.1.1.

We next describe our results in more detail.

Let $L$ be a totally real field of degree $g$ over $\mathbb{Q}$ and $p$ a rational prime, unramified in $L$. We consider the moduli space $X$ parameterizing polarized abelian varieties $\underline{A}$ with RM by $L$ and a rigid level structure prime to $p$, and the moduli space $Y$ parameterizing the same data and, in addition, a maximal "cyclic" $\mathcal{O}_{L}$-subgroup of $\underline{A}[p]$. There is a forgetful morphism $\pi: Y \rightarrow X$. Both $X$ and $Y$ are considered over the Witt vector $W(\kappa)$ of a suitable finite field $\kappa$. We let $\mathbb{X}$ and $\mathbb{Y}$ denote the minimal compactifications. See below and section $\S 2.1$.

The special fibre $\bar{X}$ of $X$ was studied in [21, where a stratification $\left\{Z_{\tau}\right\}$ indexed by subsets $\tau$ of $\mathbb{B}=\operatorname{Hom}\left(L, \overline{\mathbb{Q}}_{p}\right)$ was constructed. This stratification is intimately connected to theory of Hilbert modular forms. In particular, for every $\beta \in \mathbb{B}$ there is a Hilbert modular form $h_{\beta}-\mathrm{a}$ partial Hasse invariant - whose divisor is $Z_{\beta}$; see 2.5 . The partial Hasse invariants are a purely characteristic $p$ phenomenon, but they can be lifted locally in the Zariski topology to $X$. 
Let $\mathfrak{X}_{\text {rig }}, \mathfrak{Y}_{\text {rig }}$ denote the rigid analytic spaces associated à la Raynaud to $\mathbb{X}, \mathbb{Y}$, and $\mathfrak{X}_{\text {rig }}^{\circ}, \mathfrak{Y}_{\text {rig }}^{\circ}$ their ordinary locus. There is a kernel-of-Frobenius section $\bar{X} \rightarrow \bar{Y}$ given on points by $\underline{A} \mapsto$ $\left(\underline{A}, \operatorname{Ker}\left(\operatorname{Fr}: \underline{A} \rightarrow \underline{A}^{(p)}\right)\right)$, which extends to compactifications. We show, using a Hensel's lemma type of argument, that this section lifts to a canonical morphism $s^{\circ}: \mathfrak{X}_{\text {rig }}^{\circ} \rightarrow \mathfrak{Y}_{\text {rig }}^{\circ}$, which is a section to $\pi$.

For a point $P$ in $\mathfrak{X}_{\text {rig }}$, and $\beta \in \mathbb{B}$, let $\tilde{h}_{\beta}(P)$ denote the evaluation of a Zariski local lift of the partial Hssse invariant $h_{\beta}$ at $P$. Let $\sigma$ be the Frobenius automorphism on $\mathbb{Q}_{p}^{\text {ur }}$. One of our main theorems (Theorem 5.3.1) states the following.

Theorem A. Let $\mathcal{U} \subseteq \mathfrak{X}_{\text {rig }}$ be defined as

$$
\mathcal{U}=\left\{P: \nu\left(\tilde{h}_{\beta}(P)\right)+p \nu\left(\tilde{h}_{\sigma \circ \beta}(P)\right)<p, \forall \beta \in \mathbb{B}\right\} .
$$

There exists a section,

$$
s^{\dagger}: \mathcal{U} \rightarrow \mathfrak{Y}_{\text {rig }},
$$

extending the section $s^{\circ}$ on the ordinary locus.

In the theorem, $\nu$ is the $p$-adic valuation, normalized by $\nu(p)=1$ and truncated at 1 . In fact, comparing to Theorem 5.3.1, the reader will notice that our formulation is not the same. In 4.2 we define vector valued valuations on $\mathfrak{X}_{\text {rig }}, \mathfrak{Y}$ rig. The valuation vector $\nu(P)$ of a closed point $P$ on $\mathfrak{X}_{\text {rig }}$ takes into account the strata $Z_{\tau}$ on which $\bar{P}$, the reduction of $P$, lies. Also the valuation vector $\nu(Q)$ of a closed point $Q$ on $\mathfrak{X}_{\text {rig }}$ takes into account on which strata $\bar{Q}$ lies. A substantial part of the paper (£§2-3) is in fact devoted to defining this stratification of $\bar{Y}$ and studying it properties. Let $(\varphi, \eta)$ be a pair of subsets of $\mathbb{B}$ which is admissible (2.3; there are $3^{g}$ such strata). We define a stratification $\left\{Z_{\varphi, \eta}\right\}$ indexed by such pairs $(\varphi, \eta)$; the fundamental results concerning this stratification appear in Theorem 2.5.2. Some key facts are:

(1) $\pi\left(Z_{\varphi, \eta}\right)=Z_{\varphi \cap \eta}($ Theorem 2.6.4);

(2) every irreducible component of $Z_{\varphi, \eta}$ intersects non-trivially the finite set of points corresponding to data $\left(\underline{A}, \operatorname{Ker}\left(\operatorname{Fr}: \underline{A} \rightarrow \underline{A}^{(p)}\right)\right)$ consisting of superspecial abelian varieties with the kernel of Frobenius group scheme (Theorem 2.6.13);

(3) Every irreducible component of $\operatorname{Spf}\left(\widehat{\mathcal{O}}_{\bar{Y}}, \bar{Q}\right)$ is accounted for by a unique maximal stratum $Z_{\varphi, \eta}($ Theorem 2.5.2).

Let $\bar{Q} \in \bar{Y}(k)$ and $\bar{P}=\pi(\bar{Q})$ its image in $\bar{X}(k)$. One has natural parameters such that $\widehat{\mathcal{O}}_{X, \bar{P}} \cong W(k) \llbracket t_{\beta}: \beta \in \mathbb{B} \rrbracket$ (Equation (2.5.1)), and parameters such that $\widehat{\mathcal{O}}_{Y, \bar{Q}} \cong W(k) \llbracket\left\{x_{\beta}, y_{\beta}\right.$ : $\beta \in I\},\left\{z_{\beta}: \beta \in I^{c}\right\} \rrbracket /\left(\left\{x_{\beta} y_{\beta}-p: \beta \in I\right\}\right)$ (Equation (2.4.3)), where if $\bar{Q}$ has invariants $(\varphi, \eta)$, then $I=\ell(\varphi) \cap \eta$ and $\ell(\varphi)=\left\{\sigma^{-1} \circ \beta: \beta \in \varphi\right\}$. One of our main results, referred to as "Key Lemma" (Lemma 2.8.1) describes $\pi^{*}\left(t_{\beta}\right)$, under the induced ring homomorphism $\pi^{*}: \widehat{\mathcal{O}}_{\bar{X}, \bar{P}} \rightarrow \widehat{\mathcal{O}}_{\bar{Y}}, \bar{Q}$, for $\beta \in \varphi \cap \eta$. (It remains an interesting problem to extend this lemma to $\beta \notin \varphi \cap \eta$.) The Key lemma is crucially used in the proof of Theorem $\mathbf{A}$. It allows us to compare the valuation vectors of a point in $\mathfrak{Y}_{\text {rig }}$ and its image under $\pi$ in $\mathfrak{X}_{\text {rig }}$ as in 5.3 . Using such valuation vector calculations, we are able to isolate a union of connected components of $\pi^{-1}(\mathcal{U})$ (which we call $\mathcal{V}$ ), and prove that $\pi$ is an isomorphism when restricted to $\mathcal{V}$. The section $s^{\dagger}$ is defined as the inverse of $\left.\pi\right|_{\mathcal{V}}$. 
In 5.4 we describe the properties of the canonical subgroup. In particular, we study the property of the reduction to the kernel of Frobenius (Theorem 5.4.2) and what happens under the iteration of our construction (Theorem [5.4.3). The following theorem summarizes some aspects of these results.

Theorem B. Let $\underline{A}$ be an abelian variety with $R M$ corresponding to $P \in \mathcal{U}$. Let $r$ be an element whose valuation is $\max \left\{\nu\left(\tilde{h}_{\beta}(P)\right)\right\}_{\beta \in \mathbb{B}}$.

(1) The canonical subgroup $H$ of $\underline{A}$ reduces to $\operatorname{Ker}(\mathrm{Fr})$ modulo $p / r$.

(2) Let $C$ be a subgroup of $\underline{A}$ such that $(\underline{A}, C) \in \mathfrak{Y}_{\text {rig. }}$. Let $P^{\prime} \in \mathfrak{X}_{\text {rig }}$ correspond to $\underline{A} / C$. There is a recipe for calculating $\left\{\nu\left(\tilde{h}_{\beta}\left(P^{\prime}\right)\right)\right\}_{\beta}$ in terms of $\left\{\nu\left(\tilde{h}_{\beta}(P)\right)\right\}_{\beta}$, in particular, for determining if it affords a canonical subgroup.

Theorem B determines the p-adic geometry of the Hecke operator on the "not-too-singular" locus of $\mathfrak{Y}_{\text {rig. }}$ Theorem $\mathbf{B}$ also applies directly to deriving a theorem about higher-level canonical subgroups (Proposition 5.4.4). The results are similar to the case of elliptic curves, only that the situation is richer as the position of a point on $\mathfrak{Y}_{\text {rig }}$ is described by $g$ parameters (the components of its valuation vector) in contrast to the case of elliptic curves where there is only one parameter.

Finally, the Appendix describes a certain generalization of the moduli scheme $Y$, obtained by considering for an $\mathcal{O}_{L}$-ideal $\mathfrak{t} \mid p$, a cyclic $\mathcal{O}_{L} / \mathfrak{t}$ subgroup instead of a cyclic $\mathcal{O}_{L} /(p)$ subgroup, and briefly describes the extension of our results to this situation. The results are relevant to the construction of partial $U$ operators, indexed again by ideals $\mathfrak{t} \mid p$.

Acknowledgements. We would like to thank F. Andreatta for discussions concerning the contents of this paper.

\section{Contents}

1. Introduction

2. Moduli spaces in positive characteristic

2.1. Two formulations of a moduli problem

2.2. Some facts about Dieudonné modules

2.3. Discrete invariants for the points of $\bar{Y}$

2.4. The infinitesimal nature of $\bar{Y}$

2.5. Stratification of $\bar{Y}$

2.6. The fibres of $\pi: \bar{Y} \rightarrow \bar{X}$

2.7. The Atkin-Lehner automorphism

2.8. The infinitesimal nature of $\pi: \bar{Y} \rightarrow \bar{X}$

3. Extension to the cusps

3.1. Notation

3.2. Extension of the stratification

4. Valuations and a dissection of $\mathfrak{Y}_{\text {rig }}$

4.1. Notation

1

4.2. Valuations on $\mathfrak{X}_{\text {rig }}$ and $\mathfrak{Y}_{\text {rig }}$

4.3. The valuation cube 35

5. The canonical subgroup $\quad 36$

5.1. Some admissible open subsets of $\mathfrak{X}_{\text {rig }}$ and $\mathfrak{Y}_{\text {rig }}$

5.2. The section on the ordinary locus

5.3. The main theorem 39

5.4. Properties of the canonical subgroup 43 
6. Functoriality 46

6.1. Changing the field 47

6.2. Galois automorphisms 48

7. Appendix 49

7.1. A variant on the moduli problem 49

References

Notation. Let $p$ be a prime number, $L / \mathbb{Q}$ a totally real field of degree $g$ in which $p$ is unramified, $\mathcal{O}_{L}$ its ring of integers, $\mathfrak{d}_{L}$ the different ideal, and $N$ an integer prime to $p$. Let $L^{+}$denote the elements of $L$ that are positive under every embedding $L \hookrightarrow \mathbb{R}$. For a prime ideal $\mathfrak{p}$ of $\mathcal{O}_{L}$ dividing $p$, let $\kappa_{\mathfrak{p}}=\mathcal{O}_{L} / \mathfrak{p}, f_{\mathfrak{p}}=\operatorname{deg}\left(\kappa_{\mathfrak{p}} / \mathbb{F}_{p}\right), f=\operatorname{lcm}\left\{f_{\mathfrak{p}}: \mathfrak{p} \mid p\right\}$, and $\kappa$ a finite field with $p^{f}$ elements. We identify $\kappa_{\mathfrak{p}}$ with a subfield of $\kappa$ once and for all. Let $\mathbb{Q}_{\kappa}$ be the fraction field of $W(\kappa)$. We fix embeddings $\mathbb{Q}_{\kappa} \subset \mathbb{Q}_{p}^{\text {ur }} \subset \overline{\mathbb{Q}}_{p}$.

Let $\left[\mathrm{Cl}^{+}(L)\right]$ be a complete set of representatives for the strict (narrow) class group $\mathrm{Cl}^{+}(L)$ of $L$, chosen so that its elements are ideals $\mathfrak{a} \triangleleft \mathcal{O}_{L}$, equipped with their natural positive cone $\mathfrak{a}^{+}=\mathfrak{a} \cap L^{+}$. Let

$$
\mathbb{B}=\operatorname{Emb}\left(L, \mathbb{Q}_{\kappa}\right)=\coprod_{\mathfrak{p}} \mathbb{B}_{\mathfrak{p}}
$$

where $\mathfrak{p}$ runs over prime ideals of $\mathcal{O}_{L}$ dividing $p$, and $\mathbb{B}_{\mathfrak{p}}=\left\{\beta \in \mathbb{B}: \beta^{-1}(p W(\kappa))=\mathfrak{p}\right\}$. Let $\sigma$ denote the Frobenius automorphism of $\mathbb{Q}_{\kappa}$, lifting $x \mapsto x^{p}$ modulo $p$. It acts on $\mathbb{B}$ via $\beta \mapsto \sigma \circ \beta$, and transitively on each $\mathbb{B}_{\mathfrak{p}}$. For $S \subseteq \mathbb{B}$ we let

$$
\ell(S)=\left\{\sigma^{-1} \circ \beta: \beta \in S\right\}, \quad r(S)=\{\sigma \circ \beta: \beta \in S\},
$$

and

$$
S^{c}=\mathbb{B}-S \text {. }
$$

The decomposition

$$
\mathcal{O}_{L} \otimes_{\mathbb{Z}} W(\kappa)=\bigoplus_{\beta \in \mathbb{B}} W(\kappa)_{\beta},
$$

where $W(\kappa)_{\beta}$ is $W(\kappa)$ with the $\mathcal{O}_{L}$-action given by $\beta$, induces a decomposition,

$$
M=\bigoplus_{\beta \in \mathbb{B}} M_{\beta}
$$

on any $\mathcal{O}_{L} \otimes_{\mathbb{Z}} W(\kappa)$-module $M$.

Let $A$ be an abelian scheme over a scheme $S$, equipped with real multiplication $\iota: \mathcal{O}_{L} \rightarrow$ $\operatorname{End}_{S}(A)$. Then the dual abelian scheme $A^{\vee}$ has a canonical real multiplication, and we let $\mathcal{P}_{A}=\operatorname{Hom}_{\mathcal{O}_{L}}\left(A, A^{\vee}\right)^{\mathrm{sym}}$. It is a projective $\mathcal{O}_{L}$-module of rank 1 with a notion of positivity; the positive elements correspond to $\mathcal{O}_{L}$-equivariant polarizations.

For a $W(\kappa)$-scheme $S$ we shall denote by $\underline{A} / S$, or simply $\underline{A}$ if the context is clear, a quadruple:

$$
\underline{A} / S=(A / S, \iota, \lambda, \alpha),
$$

comprising the following data: $A$ is an abelian scheme of relative dimension $g$ over a $W(\kappa)$ scheme $S, \iota: \mathcal{O}_{L} \hookrightarrow \operatorname{End}_{S}(A)$ is a ring homomorphism. The map $\lambda$ is a polarization as in [14], namely, an isomorphism $\lambda:\left(\mathcal{P}_{A}, \mathcal{P}_{A}^{+}\right) \rightarrow\left(\mathfrak{a}, \mathfrak{a}^{+}\right)$for a representative $\left(\mathfrak{a}, \mathfrak{a}^{+}\right) \in\left[\mathrm{Cl}^{+}(L)\right]$ such that 
$A \otimes_{\mathcal{O}_{L}} \mathfrak{a} \cong A^{\vee}$. The existence of $\lambda$ is equivalent, since $p$ is unramified, to $\operatorname{Lie}(A)$ being a locally free $\mathcal{O}_{L} \otimes \mathcal{O}_{S}$-module [19]. Finally, $\alpha$ is a rigid $\Gamma_{00}(N)$-level structure, that is, $\alpha: \mu_{N} \otimes_{\mathbb{Z}} \mathfrak{d}_{L}^{-1} \rightarrow A$ is an $\mathcal{O}_{L}$-equivariant closed immersion of group schemes.

Let $X / W(\kappa)$ be the Hilbert modular scheme classifying such data $\underline{A} / S=(A / S, \iota, \lambda, \alpha)$. Let $Y / W(\kappa)$ be the Hilbert modular scheme classifying $(\underline{A} / S, H)$, where $\underline{A}$ is as above and $H$ is a finite flat isotropic $\mathcal{O}_{L}$-subgroup scheme of $A[p]$ of rank $p^{g}$, where isotropic means relative to the $\mu$-Weil pairing for some $\mu \in \mathcal{P}_{A}^{+}$of degree prime to $p$. Let

$$
\pi: Y \rightarrow X
$$

be the natural morphism, whose effect on points is $(\underline{A}, H) \mapsto \underline{A}$.

Let $\bar{X}, \mathfrak{X}, \mathfrak{X}_{\text {rig }}$ be, respectively, the special fibre of $X$, the completion of $X$ along $\bar{X}$, and the rigid analytic space associated to $\mathfrak{X}$ in the sense of Raynaud. We use similar notation $\bar{Y}, \mathfrak{Y}, \mathfrak{Y}$ rig for $Y$ and let $\pi$ denote any of the induced morphisms. These spaces have models over $\mathbb{Z}_{p}$ or $\mathbb{Q}_{p}$, denoted $X_{\mathbb{Z}_{p}}, \mathfrak{X}_{\text {rig, } \mathbb{Q}_{\mathrm{p}}}$, etc. For a point $P \in \mathfrak{X}_{\text {rig }}$ we denote by $\bar{P}=\operatorname{sp}(P)$ its specialization in $\bar{X}$, and similarly for $Y$. Let $w: Y \rightarrow Y$ be the automorphism $(\underline{A}, H) \mapsto(\underline{A} / H, A[p] / H)$. Let $s: \bar{X} \rightarrow \bar{Y}$ be the kernel-of-Frobenius section to $\pi, \underline{A} \mapsto\left(\underline{A}, \operatorname{Ker}\left(\operatorname{Fr}_{A}: A \rightarrow A^{(p)}\right)\right)$, which exists by Lemma 2.1.1. We denote $s(\bar{X})$ by $\bar{Y}_{F}$, and $w\left(\bar{Y}_{F}\right)$ by $\bar{Y}_{V}$. These are components of $\bar{Y}$, and the geometric points of $\bar{Y}_{F}$ (respectively, $\bar{Y}_{V}$ ) are the geometric points $(\underline{A}, H)$ where $H$ is $\operatorname{Ker}\left(\operatorname{Fr}_{A}\right)$ (respectively, $\operatorname{Ker}\left(\operatorname{Ver}_{A}\right)$ ). We denote the ordinary locus in $\bar{X}$ (respectively, $\left.\bar{Y}\right)$ by $\bar{X}^{\text {ord }}$ (respectively, $\left.\bar{Y}^{\text {ord }}\right)$. We define $\bar{Y}_{F}^{\text {ord }}$ to be $s\left(\bar{X}^{\text {ord }}\right)$, and $\bar{Y}_{V}^{\text {ord }}$ to be $w\left(\bar{Y}_{F}^{\text {ord }}\right)$; they are both a union of connected components of $\bar{Y}^{\text {ord }}$.

\section{Moduli spaces in positive CharaCteristiC}

2.1. Two formulations of a moduli problem. Recall that $X / W(\kappa)$ is the moduli space parameterizing data $\underline{A} / S=(A, \iota, \lambda, \alpha)$ where $S$ is a $W(\kappa)$-scheme, $A$ an abelian scheme over $S$ of relative dimension $g, \iota: \mathcal{O}_{L} \rightarrow \operatorname{End}_{S}(A)$ a ring homomorphism such that $\operatorname{Lie}(A)$ is a locally free $\mathcal{O}_{L} \otimes \mathcal{O}_{S}$ module of rank 1 (the "Rapoport condition"). The map $\alpha: \mu_{N} \otimes_{\mathbb{Z}} \mathfrak{d}_{L}^{-1} \rightarrow A$ is a rigid $\Gamma_{00}(N)$-level structure and $\lambda$ is a polarization data: an isomorphism of $\mathcal{O}_{L}$-modules with a notion of positivity, $\lambda: \mathcal{P}_{\underline{A}} \rightarrow \mathfrak{a}$, for one of the representatives $\mathfrak{a} \in\left[\mathrm{Cl}^{+}(L)\right]$ fixed above. It follows, since $p$ is unramified in $L$, that the natural morphism $A \otimes_{\mathcal{O}_{L}} \mathcal{P}_{A} \rightarrow A^{\vee}$ is an isomorphism (this fact is sometimes called the "Deligne-Pappas" condition; they introduced it in [14] in the case $p$ is possibly ramified in $L$, in replacement of the Rapoport condition). The morphism $X \rightarrow \operatorname{Spec}(W(\kappa))$ is smooth, quasi-projective, of relative dimension $g$. We let $\bar{X}=X \otimes \kappa$ denote the special fibre of $X$. It is a quasi-projective non-singular variety of dimension $g$ over $\kappa$, whose irreducible components are in bijection with $\mathrm{Cl}^{+}(L)$.

Recall also the moduli space $Y$ that parameterizes data $(\underline{A}, H) / S$, where $\underline{A} / S$ is as above and $H$ is a finite flat $\mathcal{O}_{L}$-subgroup scheme of $A[p]$ of rank $p^{g}$, isotropic relative to the $\gamma$-Weil pairing induced by a $\gamma \in \mathcal{P}_{\underline{A}}$ of degree prime to $p$,

$$
A[p] \times A[p] \stackrel{1 \times \gamma}{\cong} A[p] \times A^{\vee}[p] \longrightarrow \mu_{p}
$$


It follows that $H$ is isotropic relative to the $r \gamma$-Weil pairing, where $r \in \mathcal{O}_{L}$ is prime to $p$. Therefore, $H$ is isotropic relative to the $\delta$-Weil pairing for all $\delta \in \mathcal{P}_{\underline{A}}$ of degree prime to $p$. Since $\mathcal{P}_{\underline{A}}$ is generated as a $\mathbb{Z}$-module by such $\delta$, we conclude that $H$ is isotropic relative to the Weil pairing induced by some $\gamma \in \mathcal{P}_{\underline{A}}$ of degree prime to $p$ implies that it is isotropic relative to any Weil pairing induced by an element of $\mathcal{P}_{\underline{A}}$. Henceforth, we will simply call such $H$ isotropic.

Lemma 2.1.1. Let $\underline{A} / S$ be an object of the kind parameterized by $X$, where $S$ is a reduced $W(\kappa)$ scheme. Let $H \subseteq A[p]$ be a finite flat $\mathcal{O}_{L}$-group scheme of $\underline{A}$, which is a cyclic $\mathcal{O}_{L}$-module, where by that we mean that for every geometric point $x$ of characteristic zero of $S$ the group scheme $H_{x}$ is a cyclic $\mathcal{O}_{L}$-module, and for every geometric point $x$ of characteristic $p$ the Dieudonné module $\mathbb{D}\left(H_{x}\right)$ is a cyclic $\mathcal{O}_{L} \otimes_{\mathbb{Z}} k(x)$-module. Then $H$ is isotropic relative to any $\mathcal{O}_{L}$-polarization.

In particular, if $S$ is a characteristic $p$ scheme, and $H=\operatorname{Ker}\left(\operatorname{Fr}_{A}\right)$, then $H$ is automatically isotropic.

Proof. Let $\mu$ be an $\mathcal{O}_{L}$-polarization. The locus where $H$ is isotropic relative to $\mu$ can be viewed as the locus where $H \subseteq \mu(H)^{\perp}$ under the Weil pairing on $A[p] \times A^{\vee}[p]$, and so is a closed subset of $S$; it is enough to prove it contains every geometric point $x$ of $S$. If $x$ has characteristic zero, $H_{x}$ is a cyclic $\mathcal{O}_{L}$-module and $\mu$-induces an alternating pairing $\langle\cdot, \cdot\rangle: H_{x} \times H_{x} \rightarrow \mu_{p}$ such that $\langle\ell r, s\rangle=\langle r, \ell s\rangle$ for $\ell \in \mathcal{O}_{L}, r, s \in H_{x}$. Let $g$ be a generator of $H_{x}$; then any other element of $H_{x}$ is of the form $\ell g$ for some $\ell \in \mathcal{O}_{L}$. Then $\left\langle\ell_{1} g, \ell_{2} g\right\rangle=\left\langle\ell_{1} \ell_{2} g, g\right\rangle=\left\langle g, \ell_{1} \ell_{2} g\right\rangle=-\left\langle\ell_{1} \ell_{2} g, g\right\rangle$, and so $H_{x}$ is isotropic. If $x$ is of characteristic $p$, the argument is the same, making use of the cyclicity of the $\mathcal{O}_{L} \otimes k$ module $\mathbb{D}(A[p])$.

It remains to show that $\mathbb{D}\left(\operatorname{Ker}\left(\operatorname{Fr}_{A_{x}}\right)\right)$ is always a cyclic $\mathcal{O}_{L} \otimes k$-module. This is not automatic, and in fact it uses the Rapoport condition by which the Lie algebra of $A$ (identified with the tangent space at the identity) is a free $\mathcal{O}_{L} \otimes k$-module of rank one. On the other hand, by a result of Oda (see 92.2 ), the tangent space is, up to a twist, the Dieudonné module of the kernel of Frobenius.

We let $\bar{Y}=Y \otimes \kappa$ denote the special fibre of $Y$. It is a quasi-projective variety of dimension $g$ over $\kappa$, which, as we shall see below is highly singular and reducible (even for a fixed polarization module), although equi-dimensional. The morphism $\pi: \bar{Y} \rightarrow \bar{X}$ is proper. The space $\bar{X}$ was studied by Rapoport [32] and Goren-Oort [21] and the space $\bar{Y}$ was studied by Pappas [31, H. Stamm [34, and more recently by C.-F. Yu in [9], although we shall make no use of Yu's work here.

Our main interest in this section is in stratifications of $\bar{X}$ and $\bar{Y}$ and how they relate via the morphism $\pi: \bar{Y} \rightarrow \bar{X}$, but first we provide another interpretation of $Y$.

Lemma 2.1.2. The moduli space $Y$ is also the moduli space of data $(f: \underline{A} \rightarrow \underline{B})$, where $\underline{A}=$ $\left(A, \iota_{A}, \lambda_{A}, \alpha_{A}\right), \underline{B}=\left(B, \iota_{B}, \lambda_{B}, \alpha_{B}\right)$ are polarized abelian varieties with real multiplication and $\Gamma_{00}(N)$-structure, and $f$ is an $\mathcal{O}_{L}$-isogeny, killed by $p$ and of degree $p^{g}$, such that $f^{*} \mathcal{P}_{B}=p \mathcal{P}_{A}$. (In particular, $\underline{A}$ and $\underline{B}$ have isomorphic polarization modules).

Proof. Let $(A, H)$ be as above. We define $\underline{B}=\left(B, \iota_{B}, \lambda_{B}, \alpha_{B}\right)$ to be $A / H$ with the naturally induced real multiplication by $\mathcal{O}_{L}$ and $\Gamma_{00}(N)$-level structure; $\lambda_{B}$ will be defined below. Let $f: A \rightarrow A / H$ denote the natural isogeny, and let $f^{t}: A / H \rightarrow A$ be the unique isogeny such that $f^{t} \circ f=[p]_{A}$; its kernel is $A[p] / H$. The short exact sequence $0 \rightarrow A[p] / H \rightarrow A / H \stackrel{f^{t}}{\rightarrow} A \rightarrow 0$ 
induces a short exact sequence

$$
0 \longrightarrow(A[p] / H)^{\vee} \longrightarrow A^{\vee} \stackrel{\left(f^{t}\right)^{\vee}}{\longrightarrow}(A / H)^{\vee} \longrightarrow 0 .
$$

Since the annihilator of $H$ under the Weil pairing $A[p] \times A^{\vee}[p] \rightarrow \mu_{p}$ is $(A[p] / H)^{\vee}$, it follows that $H$ is isotropic if and only if for any $\gamma \in \mathcal{P}_{A}$, we have $\gamma(H) \subseteq(A[p] / H)^{\vee}$. For such $H$ and $\gamma \in \mathcal{P}_{A}$, we have a commutative diagram:

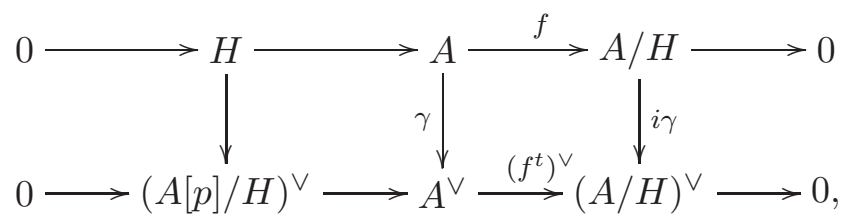

where we have denoted by $i \gamma$ the map $A / H \rightarrow(A / H)^{\vee}$ appearing in the diagram; $\gamma \mapsto i \gamma$ is an $\mathcal{O}_{L}$-linear homomorphism $i: \mathcal{P}_{A} \rightarrow \mathcal{P}_{B}$. It follows from this definition that $i \gamma \circ f=\left(f^{t}\right)^{\vee} \circ \gamma$. In particular,

$$
\operatorname{deg}(i \gamma)=\operatorname{deg}(\gamma)
$$

As a result, $i$ is injective. For every $\gamma \in \mathcal{P}_{A}$ we have

$$
f^{*}(i \gamma)=f^{\vee} \circ i \gamma \circ f=f^{\vee} \circ\left(f^{t}\right)^{\vee} \circ \gamma=\left(f^{t} \circ f\right)^{\vee} \circ \gamma=p \gamma
$$

Therefore, the composition $f^{*} \circ i: \mathcal{P}_{A} \rightarrow \mathcal{P}_{A}$ is multiplication by $p$. In particular, we have $f^{*}\left(\mathcal{P}_{B}\right) \supseteq p \mathcal{P}_{A}$. We now show that $f^{*}\left(\mathcal{P}_{B}\right) \subseteq p \mathcal{P}_{A}$. To this end, consider the map $f^{t}: A / H \rightarrow A$ of kernel $A[p] / H$. Let $\gamma \in \mathcal{P}_{A}$ be a polarization of degree prime to $p$. Hence, the polarization $i \gamma$ is also of degree prime to $p$. To show that $A[p] / H$ is isotropic it is enough to show it is isotropic relative to $i \gamma$, that is, $i \gamma(A[p] / H)=H^{\vee}\left(H^{\vee}\right.$ is naturally identified with the annihilator of $A[p] / H$ in $\left.(A / H)^{\vee}\right)$. And indeed, $i \gamma(A[p] / H)=i \gamma \circ f(A[p])=\left(f^{t}\right)^{\vee} \gamma(A[p])=\left(f^{t}\right)^{\vee}\left(A^{\vee}[p]\right)=$ $A^{\vee}[p] /(A[p] / H)^{\vee}=H^{\vee}$. We may now apply the same arguments made above and conclude that there is an $\mathcal{O}_{L}$-linear map $j: \mathcal{P}_{B} \rightarrow \mathcal{P}_{A}$, satisfying $\left(f^{t}\right)^{*} \circ j=p$.

Let $\gamma \in \mathcal{P}_{B}$. We claim that $p \cdot j \gamma=f^{*} \gamma$ (and so $f^{*}\left(\mathcal{P}_{B}\right) \subseteq p \mathcal{P}_{A}$ holds). To show that, it is enough to show that $\left(f^{t}\right)^{*} p j \gamma=\left(f^{t}\right)^{*} f^{*} \gamma$. The right hand side is $p^{*} \gamma=p^{2} \gamma$, while $\left(f^{t}\right)^{*} p j \gamma=$ $p\left(f^{t}\right)^{*} j \gamma=p^{2} \gamma$.

We now define,

$$
\lambda_{B}: \mathcal{P}_{B} \stackrel{\cong}{\longrightarrow} \mathfrak{a}, \quad \lambda_{B}=\frac{1}{p} \lambda_{A} \circ f^{*} .
$$

It remains to show that the Deligne-Pappas condition holds for $\underline{B}$. By [3, Proposition 3.1], it is enough to show that for every prime $\ell$ (including $\ell=p$ ), there is an element $\gamma^{\prime}$ of $\mathcal{P}_{B}$ of degree prime to $\ell$. Let $\gamma \in \mathcal{P}_{A}$ be an element of degree prime to $\ell$, which exists since $\underline{A}$ satisfies the said condition, and let $\gamma^{\prime}=i \gamma$.

Let $f: \underline{A} \rightarrow \underline{B}$ be an isogeny as in the statement of the lemma and $H=\operatorname{Ker}(f)$. We only need to show that $H$ is isotropic relative to $\mathcal{P}_{A}$. Let $\gamma \in \mathcal{P}_{A}$; to show that $\gamma(H) \subseteq(A[p] / H)^{\vee}$, is to show that the composition $\left(f^{t}\right)^{\vee} \circ \gamma(H)=0$. Now, applying $f^{*}$ to $f^{*} \mathcal{P}_{B}=p \mathcal{P}_{A}$ we find $\left(f^{t}\right)^{*} \mathcal{P}_{A}=p \mathcal{P}_{B}$. Hence, $\left(f^{t}\right)^{\vee} \circ \gamma \circ f^{t}=\left(f^{t}\right)^{*} \gamma=\delta p=\delta \circ f \circ f^{t}$ for some $\delta \in \mathcal{P}_{B}$. Therefore, $\left(f^{t}\right)^{\vee} \circ \gamma(H)=\delta \circ f(H)=0$. 
2.2. Some facts about Dieudonné modules. Let $k$ be a perfect field of positive characteristic $p$. We let $\mathbb{D}$ denote the contravariant Dieudonné functor, $G \mapsto \mathbb{D}(G)$, from finite commutative $p$-primary group schemes $G$ over $k$, to finite length $W(k)$-modules $M$ equipped with two maps $\operatorname{Fr}: M \rightarrow M$, Ver: $M \rightarrow M$, such that $\operatorname{Fr}(\alpha m)=\sigma(\alpha) \operatorname{Fr}(m), \operatorname{Ver}(\sigma(\alpha) m)=\alpha \operatorname{Ver}(m)$ for $\alpha \in W(k), m \in M$ and $\mathrm{Fr} \circ \mathrm{Ver}=\operatorname{Ver} \circ \mathrm{Fr}=[p]$. This functor is an anti-equivalence of categories and commutes with base change. It follows that if $G$ has rank $p^{\ell}$ the length of $\mathbb{D}(G)$ is $\ell$.

Given a morphism of group schemes $f: G \rightarrow H$ we find that

$$
\mathbb{D}(\operatorname{Ker}(f))=\mathbb{D}(G) / \mathbb{D}(f)(\mathbb{D}(H)),
$$

where, in fact, $\mathbb{D}(f)(\mathbb{D}(H))$ depends only on $f(G)$.

Suppose $f, g: G \rightarrow H$ are two morphisms. By considering the morphism $(f, g): G \rightarrow H \times H$ we find that

$$
\mathbb{D}(\operatorname{Ker}(f) \cap \operatorname{Ker}(g))=\mathbb{D}(G) / \mathbb{D}((f, g))(\mathbb{D}(H \times H))=\mathbb{D}(G) /[\mathbb{D}(f)(\mathbb{D}(H))+\mathbb{D}(g)(\mathbb{D}(H))] .
$$

On the other hand, since $\operatorname{Ker}(f) \cap \operatorname{Ker}(g)=\operatorname{Ker}\left(\left.f\right|_{\operatorname{Ker}(g)}\right)$, we have

$$
\mathbb{D}(\operatorname{Ker}(f) \cap \operatorname{Ker}(g))=\mathbb{D}(\operatorname{Ker}(g)) / \mathbb{D}(f)(\mathbb{D}(H)),
$$

where here we may replace $H$ by any subgroup scheme containing $f(\operatorname{Ker}(g))$, if we wish.

- The Frobenius morphism $\operatorname{Fr}_{G}: G \rightarrow G^{(p)}$ induces a linear map of Dieudonné modules

$$
\mathbb{D}\left(\operatorname{Fr}_{G}\right): \mathbb{D}\left(G^{(p)}\right) \rightarrow \mathbb{D}(G),
$$

and, using that $\mathbb{D}\left(G^{(p)}\right)=\mathbb{D}\left(G \otimes_{W(k)} W(k)\right)=\mathbb{D}(G) \otimes_{W(k)} W(k)$, which is a (right) $W(k)$-module via $(m \otimes 1) s=m \otimes s=\sigma^{-1}(s) \cdot m \otimes 1$, we get the $\sigma$-linear map

$$
\operatorname{Fr}: \mathbb{D}(G) \rightarrow \mathbb{D}(G), \quad \operatorname{Fr}(t m)=\sigma(t) \operatorname{Fr}(m),
$$

via the inclusion $\mathbb{D}(G) \rightarrow \mathbb{D}(G) \otimes_{W(k)} W(k)$; it has the same image as $\mathbb{D}\left(\operatorname{Fr}_{A}\right)$. Similarly, the Verschiebung morphism $\operatorname{Ver}_{G}: G \rightarrow G^{(1 / p)}$ induces the $\sigma^{-1}$-linear map Ver: $\mathbb{D}(G) \rightarrow \mathbb{D}(G)$.

- Let $A / k$ be a $g$-dimensional abelian variety and $A[p]$ its $p$-torsion subgroup. Then $\mathbb{D}(A[p])$ is a vector space of dimension $2 g$ over $k$. The group schemes $\operatorname{Ker}\left(\operatorname{Fr}_{A}\right), \operatorname{Ker}\left(\operatorname{Ver}_{A}\right)$ are subgroups of $A[p]$ of rank $p^{g}$, where $\operatorname{Fr}_{A}: A \rightarrow A^{(p)}, \operatorname{Ver}_{A}: A \rightarrow A^{(1 / p)}$, are the usual morphisms. In fact, $A \mapsto \operatorname{Ker}\left(\operatorname{Fr}_{A}\right)$ is a functor from abelian varieties over $k$ to finite commutative group schemes, as follows from the following commutative diagram

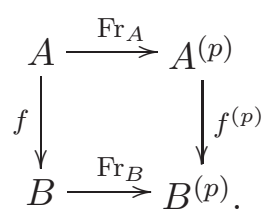

(Similarly for $A \mapsto \operatorname{Ker}\left(\operatorname{Ver}_{A}\right)$.) In particular, any endomorphism of $A$ induces endomorphisms on $\operatorname{Ker}\left(\operatorname{Fr}_{A}\right), \operatorname{Ker}\left(\operatorname{Ver}_{A}\right)$ and on

$$
\alpha(A):=\operatorname{Ker}\left(\operatorname{Fr}_{A}\right) \cap \operatorname{Ker}\left(\operatorname{Ver}_{A}\right) .
$$


Note that we have,

$$
\mathbb{D}\left(\operatorname{Ker}\left(\operatorname{Fr}_{A}\right)\right)=\mathbb{D}(A[p]) / \mathbb{D}\left(\operatorname{Fr}_{A}\right)\left(\mathbb{D}\left(A^{(p)}[p]\right)\right)=\mathbb{D}(A[p]) / \operatorname{Fr}(\mathbb{D}(A[p])),
$$

and similarly for Verschiebung,

$$
\mathbb{D}\left(\operatorname{Ker}\left(\operatorname{Ver}_{A}\right)\right)=\mathbb{D}(A[p]) / \mathbb{D}\left(\operatorname{Ver}_{A}\right)\left(\mathbb{D}\left(A^{(1 / p)}[p]\right)\right)=\mathbb{D}(A[p]) / \operatorname{Ver}(\mathbb{D}(A[p])) .
$$

Rank considerations give that $\operatorname{Fr}(\mathbb{D}(A[p]))=\operatorname{Ker}(\operatorname{Ver}: \mathbb{D}(A[p]) \rightarrow \mathbb{D}(A[p]))$ and $\operatorname{Ver}(\mathbb{D}(A[p]))=$ $\operatorname{Ker}(\operatorname{Fr}: \mathbb{D}(A[p]) \rightarrow \mathbb{D}(A[p]))$.

- The Dieudonné modules of $\operatorname{Ker}\left(\operatorname{Fr}_{A}\right), \operatorname{Ker}\left(\operatorname{Ver}_{A}\right)$ and $\operatorname{Ker}(A[p])$ are linked to cohomology by the following commutative diagram [30]:

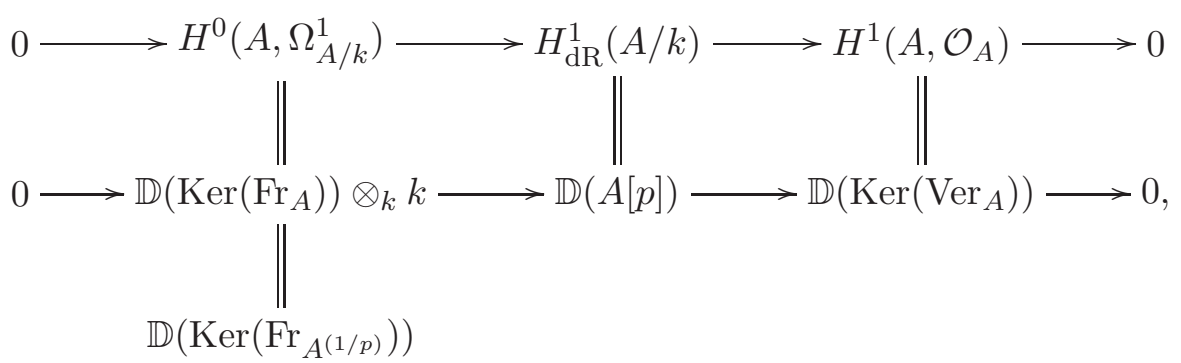

functorially in $A$; in the tensor $\operatorname{sign} \otimes_{k} k, k$ is viewed a left $k$-module relative to the map $a \mapsto a^{1 / p}$. In fact, once one has established a canonical isomorphism $H_{\mathrm{dR}}^{1}(A / k)=\mathbb{D}(A[p])$ the rest follows from the theory above.

- Suppose that $A$ has real multiplication, $\iota: \mathcal{O}_{L} \hookrightarrow \operatorname{End}_{k}(A)$. Then we have a decomposition

$$
\mathbb{D}(A[p])=\oplus_{\beta \in \mathbb{B}} \mathbb{D}(A[p])_{\beta},
$$

where $\mathbb{D}(A[p])_{\beta}$ is a two dimensional vector space over $k$ on which $\mathcal{O}_{L}$ acts via $\beta$. The maps $\mathrm{Fr}$ and Ver act thus:

$$
\text { Fr: } \mathbb{D}(A[p])_{\beta} \longrightarrow \mathbb{D}(A[p])_{\sigma \circ \beta}, \quad \text { Ver }: \mathbb{D}(A[p])_{\sigma \circ \beta} \longrightarrow \mathbb{D}(A[p])_{\beta} .
$$

Now suppose that $(A, \iota)$ satisfy that $\operatorname{Lie}(A)$ is a locally free $\mathcal{O}_{L}$-module. It then follows easily that for every $\beta \in \mathbb{B}$ the $\beta$ component of the Dieudonné submodules $\operatorname{Ker}(\mathrm{Fr})=\operatorname{Im}(\mathrm{Ver}), \operatorname{Im}(\mathrm{Fr})=$ $\operatorname{Ker}(\operatorname{Ver})$ of $\mathbb{D}(A[p])$ are one-dimensional over $k$, and, similarly

$$
\mathbb{D}\left(\operatorname{Ker}\left(\operatorname{Fr}_{A}\right)\right)=\oplus_{\beta \in \mathbb{B}} \mathbb{D}\left(\operatorname{Ker}\left(\operatorname{Fr}_{A}\right)\right)_{\beta}, \quad \mathbb{D}\left(\operatorname{Ker}\left(\operatorname{Ver}_{A}\right)\right)=\oplus_{\beta \in \mathbb{B}} \mathbb{D}\left(\operatorname{Ker}\left(\operatorname{Ver}_{A}\right)\right)_{\beta},
$$

is a decomposition into one dimensional $k$-vector spaces.

2.3. Discrete invariants for the points of $\bar{Y}$. Let $k \supseteq \kappa$ be a field. For $f: \underline{A} \rightarrow \underline{B}$ defined over $k$ as in Lemma 2.1 .2 there is a unique $\mathcal{O}_{L}$-isogeny $f^{t}: \underline{B} \rightarrow \underline{A}$ such that

$$
f^{t} \circ f=\left[p_{A}\right], \quad f \circ f^{t}=\left[p_{B}\right] .
$$


(For the relation between $f^{t}$ and the dual isogeny $f^{\vee}$ see Diagram (2.3.4).) We have induced homomorphisms:

$$
\begin{aligned}
& \bigoplus_{\beta \in \mathbb{B}} \operatorname{Lie}(f)_{\beta}: \bigoplus_{\beta \in \mathbb{B}} \operatorname{Lie}(\underline{A})_{\beta} \longrightarrow \bigoplus_{\beta \in \mathbb{B}} \operatorname{Lie}(\underline{B})_{\beta}, \\
& \bigoplus_{\beta \in \mathbb{B}} \operatorname{Lie}\left(f^{t}\right)_{\beta}: \bigoplus_{\beta \in \mathbb{B}} \operatorname{Lie}(\underline{B})_{\beta} \longrightarrow \bigoplus_{\beta \in \mathbb{B}} \operatorname{Lie}(\underline{A})_{\beta} .
\end{aligned}
$$

We note that since $\operatorname{Lie}(\underline{A})$ is a free $\mathcal{O}_{L} \otimes k$-module, $\operatorname{Lie}(\underline{A})_{\beta}$ is a one dimensional $k$-vector space. Using these decompositions we define several discrete invariants associated to the data $(f: \underline{A} \rightarrow \underline{B})$. We let

$$
\begin{aligned}
& \varphi(f)=\varphi(\underline{A}, H)=\left\{\beta \in \mathbb{B}: \operatorname{Lie}(f)_{\sigma^{-1} \circ \beta}=0\right\}, \\
& \eta(f)=\eta(\underline{A}, H)=\left\{\beta \in \mathbb{B}: \operatorname{Lie}\left(f^{t}\right)_{\beta}=0\right\}, \\
& I(f)=I(\underline{A}, H)=\ell(\varphi(f)) \cap \eta(f)=\left\{\beta \in \mathbb{B}: \operatorname{Lie}(f)_{\beta}=\operatorname{Lie}\left(f^{t}\right)_{\beta}=0\right\} .
\end{aligned}
$$

The elements of $I(\underline{A}, H)$ are the critical indices of [34].

Definition 2.3.1. Let $(\varphi, \eta)$ be a pair of subsets of $\mathbb{B}$. We say that $(\varphi, \eta)$ is an admissible pair if $\ell\left(\varphi^{c}\right) \subseteq \eta$. Given another admissible pair $\left(\varphi^{\prime}, \eta^{\prime}\right)$ we say that

$$
\left(\varphi^{\prime}, \eta^{\prime}\right) \geq(\varphi, \eta)
$$

if both inclusions $\varphi^{\prime} \supseteq \varphi, \eta^{\prime} \supseteq \eta$ hold.

Proposition 2.3.2. (1) Let $(\varphi, \eta)$ be an admissible pair. Then $r\left(\eta^{c}\right) \subseteq \varphi$, and this identity is equivalent to the admissibility of $(\varphi, \eta)$. Let $I=\ell(\varphi) \cap \eta$ then

$$
\varphi=r\left(\eta^{c}\right) \coprod r(I), \quad \eta=\ell\left(\varphi^{c}\right) \coprod I .
$$

(2) There are $3^{g}$ admissible pairs.

(3) Let $k \supseteq \kappa$ be a field. Let $(\underline{A}, H)$ correspond to a k-rational point of $\bar{Y}$ then $(\varphi(\underline{A}, H), \eta(\underline{A}, H))$ is an admissible pair.

Proof. The first part is elementary. By first choosing $\varphi$ and then choosing $\eta$ subject to the admissibility condition, the second part follows from the identity $\sum_{i=0}^{g}\left(\begin{array}{c}g \\ i\end{array}\right) 2^{i}=(1+2)^{g}=3^{g}$. Consider the third part. The condition $f \circ f^{t}=[p]$ implies for every $\beta \in \mathbb{B}$ the equality $\operatorname{Lie}(f)_{\beta} \circ$ $\operatorname{Lie}\left(f^{t}\right)_{\beta}=0$. That means that if $\beta \notin \eta(f)$ then $\operatorname{Lie}(f)_{\beta}=0$ and so $\sigma \circ \beta \in \varphi(f)$, that is $r\left(\eta(f)^{c}\right) \subseteq \varphi(f)$.

Let $k$ be a prefect field of characteristic $p$. Given $\underline{A} / k$ the type of $\underline{A}$ is defined by

$$
\tau(\underline{A})=\left\{\beta \in \mathbb{B}: \mathbb{D}\left(\operatorname{Ker}\left(\operatorname{Fr}_{A}\right) \cap \operatorname{Ker}\left(\operatorname{Ver}_{A}\right)\right)_{\beta} \neq 0\right\} .
$$

One may also define the type $\tau(\underline{A})$ as $\left\{\sigma \circ \beta: \operatorname{Ker}\left(\operatorname{Fr}_{A}: H^{1}\left(A, \mathcal{O}_{A}\right) \rightarrow H^{1}\left(A, \mathcal{O}_{A}\right)\right)_{\beta} \neq 0\right\}$. It is an exercise to check that this definition is equivalent to the one given above. The virtue of this alternative definition is that it also holds when $A$ is defined over a non-perfect field $k$, and is stable under base change. Thus, if $k^{\prime}$ is a perfect field containing $k$ and $\underline{A}$ is defined over $k$, $\tau(\underline{A})=\tau\left(\underline{A} \otimes_{k} k^{\prime}\right)$, under any definition of the right hand side.

Basic properties of Dieudonné modules discussed in $\$ 2.2$ imply that

$$
\mathbb{D}\left(\operatorname{Ker}\left(\operatorname{Fr}_{A}\right) \cap \operatorname{Ker}\left(\operatorname{Ver}_{A}\right)\right)=\mathbb{D}(A[p]) /\left(\operatorname{Im} \mathbb{D}\left(\operatorname{Fr}_{A}\right)+\operatorname{Im} \mathbb{D}\left(\operatorname{Ver}_{A}\right)\right)=\mathbb{D}(A[p]) /(\operatorname{Im} \operatorname{Fr}+\operatorname{Im} \operatorname{Ver}) .
$$


Since $\mathbb{D}(A[p])_{\beta}$ is a two dimensional $k$-vector space and both $\operatorname{Im}\left(\mathbb{D}\left(\operatorname{Fr}_{A}\right)\right)_{\beta}$ and $\operatorname{Im}\left(\mathbb{D}\left(\operatorname{Ver}_{A}\right)\right)_{\beta}$ are one dimensional, the first assertion of the following lemma holds.

Lemma 2.3.3. Let $\underline{A}$ be as above and $(f: \underline{A} \rightarrow \underline{B})$ a k-rational point of $\bar{Y}$.

(1) $\beta \in \tau(\underline{A})$ if and only if one of the following equivalent statements hold:

(a) $\operatorname{Im}\left(\mathbb{D}\left(\operatorname{Fr}_{A}\right)\right)_{\beta}=\operatorname{Im}\left(\mathbb{D}\left(\operatorname{Ver}_{A}\right)\right)_{\beta}$.

(b) $\operatorname{Im}(\operatorname{Fr})_{\beta}=\operatorname{Im}(\operatorname{Ver})_{\beta}$.

(c) $\operatorname{Ker}(\operatorname{Fr})_{\beta}=\operatorname{Ker}(\operatorname{Ver})_{\beta}$.

(2) $\beta \in \varphi(f) \Longleftrightarrow \operatorname{Im}\left(\mathbb{D}\left(\operatorname{Fr}_{A}\right)\right)_{\beta}=\operatorname{Im}(\mathbb{D}(f))_{\beta}$.

(3) $\beta \in \eta(f) \Longleftrightarrow \operatorname{Im}\left(\mathbb{D}\left(\operatorname{Ver}_{A}\right)\right)_{\beta}=\operatorname{Im}(\mathbb{D}(f))_{\beta}$.

(All Dieudonné submodules appearing above are inside $\mathbb{D}(A[p])$.)

Proof. The first assertion was already proven above. To prove (2) we recall the following commutative diagram:

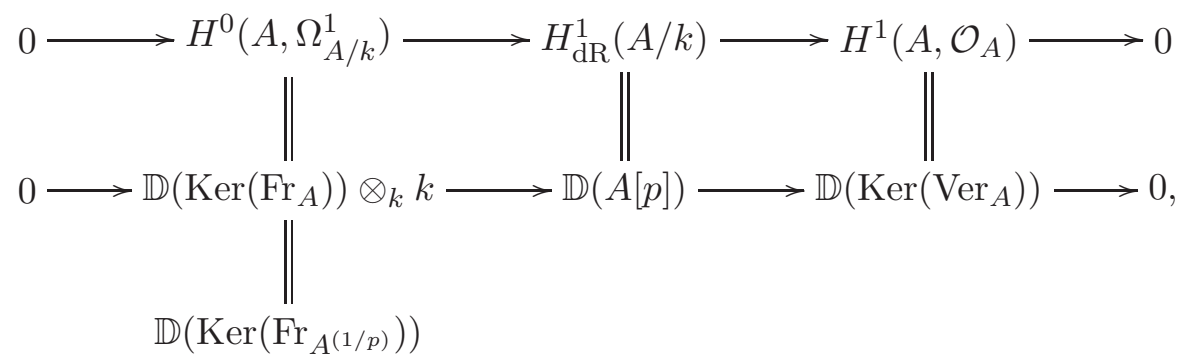

which is functorial in $A$. The map $\operatorname{Lie}(f): \operatorname{Lie}(A) \rightarrow \operatorname{Lie}(B)$ induces the map

$$
f^{*}: \operatorname{Lie}(B)^{*}=H^{0}\left(B, \Omega_{B / k}^{1}\right) \rightarrow \operatorname{Lie}(A)^{*}=H^{0}\left(A, \Omega_{A / k}^{1}\right)
$$

which is precisely the pull-back map $f^{*}$ on differentials. The map $f^{*}$ has isotypic decomposition relative to the $\mathcal{O}_{L} \otimes k$-module structure.

Now, $\beta \in \varphi(f) \Longleftrightarrow \operatorname{Lie}(f)_{\sigma^{-1} \circ \beta}=0 \Longleftrightarrow f_{\sigma^{-1} \circ \beta}^{*}=0$. Via the identifications in the above diagram, the map $f^{*}$ can also be viewed as a map

$$
f^{*}: \mathbb{D}\left(\operatorname{Ker}\left(\operatorname{Fr}_{B^{(1 / p)}}\right)\right) \rightarrow \mathbb{D}\left(\operatorname{Ker}\left(\operatorname{Fr}_{A^{(1 / p)}}\right)\right),
$$

which is equal to the linear map $\mathbb{D}\left(\left.f^{(1 / p)}\right|_{\operatorname{Ker}\left(\operatorname{Fr}_{A}(1 / p)\right.}\right)$. So,

$$
\begin{aligned}
f_{\sigma^{-1} \circ \beta}^{*}=0 & \left.\Longleftrightarrow \mathbb{D}\left(\left.f^{(1 / p)}\right|_{\operatorname{Ker}\left(\operatorname{Fr}_{A}(1 / p)\right.}\right)\right)_{\sigma^{-1} \circ \beta}=0 \\
& \Longleftrightarrow \mathbb{D}\left(\left.f\right|_{\operatorname{Ker}\left(\operatorname{Fr}_{A}\right)}\right)_{\beta}=0 .
\end{aligned}
$$

We therefore have,

$$
\beta \in \varphi(f) \Longleftrightarrow \mathbb{D}\left(\left.f\right|_{\operatorname{Ker}\left(\operatorname{Fr}_{A}\right)}\right)_{\beta}=0 .
$$

Now, $\mathbb{D}\left(\left.f\right|_{\operatorname{Ker}\left(\operatorname{Fr}_{A}\right)}\right)_{\beta}=0$ if and only if $\left[\mathbb{D}\left(\operatorname{Ker}\left(\operatorname{Fr}_{A}\right)\right) / \mathbb{D}(f)\left(\mathbb{D}\left(\operatorname{Ker} \operatorname{Fr}_{B}\right)\right)\right]_{\beta} \neq 0$ and that is equivalent to $\mathbb{D}(A[p])_{\beta} /\left[\mathbb{D}(f)(\mathbb{D}(B[p]))+\mathbb{D}\left(\operatorname{Fr}_{A}\right)\left(\mathbb{D}\left(A^{(p)}[p]\right)\right)\right]_{\beta} \neq 0$. By considering dimensions over $k$ we see that this happens if and only if $\operatorname{Im}(\mathbb{D}(f))_{\beta}=\operatorname{Im}\left(\mathbb{D}\left(\operatorname{Fr}_{A}\right)\right)_{\beta}$, as the lemma states.

We first show that

$$
\operatorname{Lie}\left(f^{t}\right)_{\beta}=0 \Longleftrightarrow H^{1}(f)_{\beta}=0 .
$$


Let $\gamma \in \mathcal{P}_{A}$ be an isogeny of degree prime to $p$. Let $i \gamma \in \mathcal{P}_{B}$ be the isogeny constructed in Diagram (2.1.1). Since $f^{\vee} \circ i \gamma f=f^{*} \gamma=p \gamma=\gamma p=\gamma \circ f^{t} \circ f$, it follows that $f^{\vee} \circ i \gamma=\gamma \circ f^{t}$ and so the following diagram is commutative:

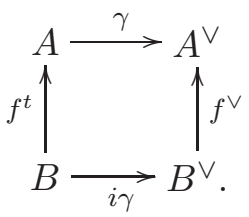

Applying Lie $(\cdot)_{\beta}$ to the diagram, we obtain

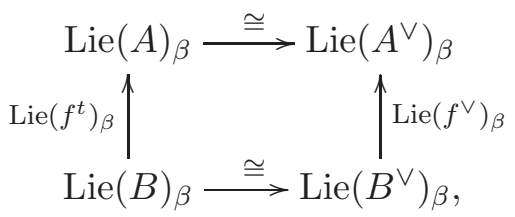

and, hence,

$$
\operatorname{Lie}\left(f^{t}\right)_{\beta}=0 \Longleftrightarrow \operatorname{Lie}\left(f^{\vee}\right)_{\beta}=0 .
$$

Since we have a commutative diagram:

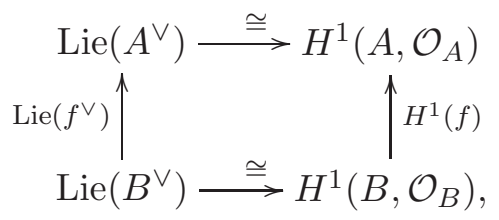

where we can pass to $\beta$-components, we conclude that $\operatorname{Lie}\left(f^{t}\right)_{\beta}=0 \Longleftrightarrow H^{1}(f)_{\beta}=0$.

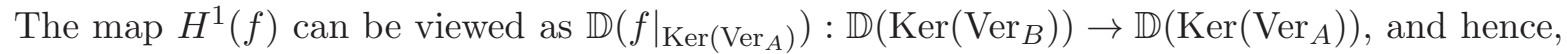
$H^{1}(f)_{\beta}=0$ if and only if $\mathbb{D}\left(\left.f\right|_{\operatorname{Ker}\left(\operatorname{Ver}_{A}\right)}\right)_{\beta}=0$. This is equivalent to

$$
\mathbb{D}(A[p])_{\beta} /\left[\mathbb{D}(f)(\mathbb{D}(B[p]))+\mathbb{D}\left(\operatorname{Ver}_{A}\right)\left(\mathbb{D}\left(A^{(p)}[p]\right)\right)\right]_{\beta} \neq 0 .
$$

Dimension considerations show that this happens if and only if $\operatorname{Im}(\mathbb{D}(f))_{\beta}=\operatorname{Im}\left(\mathbb{D}\left(\operatorname{Ver}_{A}\right)\right)_{\beta}$.

Corollary 2.3.4. The following inclusions hold.

(1) $\varphi(\underline{A}, H) \cap \eta(\underline{A}, H) \subseteq \tau(\underline{A})$.

(2) $\varphi(\underline{A}, H)^{c} \cap \eta(\underline{A}, H) \subseteq \tau(\underline{A})^{c}$.

(3) $\varphi(\underline{A}, H) \cap \eta(\underline{A}, H)^{c} \subseteq \tau(\underline{A})^{c}$.

If we denote for two sets $S, T$ their symmetric difference by $S \triangle T=(S-T) \cup(T-S)$, we can then formulate these statements as

$$
\varphi(\underline{A}, H) \cap \eta(\underline{A}, H) \subseteq \tau(\underline{A}), \quad \tau(\underline{A}) \subseteq[\varphi(\underline{A}, H) \triangle \eta(\underline{A}, H)]^{c} .
$$


2.4. The infinitesimal nature of $\bar{Y}$. In 34] Stamm studied the completed local ring of $Y$ at a closed point $\bar{Q}$ of its special fiber and concluded Theorem 2.4.1 below. Since then, local deformation theory of abelian varieties, and in particular the theory of local models, have developed and we have found it more enlightening to describe Stamm's result in that language. Our approach is not different in essence from Stamm's, but results such as Lemma 2.4.2 become more transparent in our description. Our focus is on the completed local rings of $\bar{Y}$ at a point $\bar{Q}$ defined over a perfect field $k \supseteq \kappa$ of characteristic $p$.

As in [14], one constructs a morphism from a Zariski-open neighborhood $T \subset \bar{Y}$ of $\bar{Q}$ to the Grassmann variety $\mathbf{G}$ associated to the data: $H=\left(\mathcal{O}_{L} \otimes k\right)^{2}$, two free $\mathcal{O}_{L} \otimes k$-sub-modules of $H$, say $W_{1}, W_{2}$, such that under the $\mathcal{O}_{L} \otimes k$ map $h: H \rightarrow H$ given by $(x, y) \mapsto(y, 0)$, we have $h\left(W_{1}\right) \subseteq W_{2}, h\left(W_{2}\right) \subseteq W_{1}$. Notice that we can perform the usual decomposition according to $\mathcal{O}_{L}$-eigenspaces to get

$$
h=\oplus_{\beta} h_{\beta}: \oplus_{\beta} k_{\beta}^{2} \rightarrow \oplus_{\beta} k_{\beta}^{2},
$$

such that each $h_{\beta}$ is the linear transformation corresponding to two-by-two matrix $M=\left(\begin{array}{ll}0 & 1 \\ 0 & 0\end{array}\right)$. Furthermore, $W_{i}=\oplus_{\beta}\left(W_{i}\right)_{\beta}$, and $\left(W_{i}\right)_{\beta}$ is a one-dimensional $k$-vector space contained in $k^{2}$. We have $M W_{1} \subseteq W_{2}, M W_{2} \subseteq W_{1}$.

The basis for this construction is Grothendieck's crystalline theory [23]; see also [13]. Let $(f: \underline{A} \rightarrow \underline{B})$ correspond to $\bar{Q}$. The $\mathcal{O}_{L} \otimes k$-module $H$ is isomorphic to $H_{\mathrm{dR}}^{1}(\underline{A} / k)$. By the elementary divisors theorem, we can then identify $H_{\mathrm{dR}}^{1}(\underline{B} / k)$ with $H$, and possibly adjust the identification of $H_{\mathrm{dR}}^{1}(\underline{A} / k)$ with $H$, such that the induced maps $f^{*}$ and $\left(f^{t}\right)^{*}$ are both the map $h$ defined above. Let $W_{A}=H^{0}\left(A, \Omega_{A / k}^{1}\right)=\operatorname{Lie}(A)^{*} \subset H$ be the Hodge flitration, and similarly for $W_{B}$. Then we have $h\left(W_{A}\right) \subseteq W_{B}, h\left(W_{B}\right) \subseteq W_{A}$, and so we get a point $\mathbf{Q}$ of the Grassmann variety G described above. Let $\mathcal{O}=\widehat{\mathcal{O}}_{\bar{Y}, \bar{Q}}$ and $\mathfrak{m}$ be the maximal ideal. By Grothendieck's theory, the deformations of $(f: \underline{A} \rightarrow \underline{B})$ over $R:=\mathcal{O} / \mathfrak{m}^{p}$ (which carries a canonical divided power structure) are given by deformation of the Hodge filtration over that quotient ring. Namely, are in bijection with free, direct summands, $\mathcal{O}_{L} \otimes R$-modules $\left(W_{A}^{R}, W_{B}^{R}\right)$ of rank one of $H \otimes_{k} \mathcal{O} / \mathfrak{m}^{p}=\left(\mathcal{O}_{L} \otimes R\right)^{2}$ such that $h\left(W_{A}^{R}\right) \subseteq W_{B}^{R}, h\left(W_{B}^{R}\right) \subseteq W_{A}^{R}$, and $W_{A}^{R} \otimes k=W_{A}, W_{B}^{R} \otimes k=W_{B}$. This, by the universal property of the Grassmann variety is exactly $\widehat{\mathcal{O}}_{\mathbf{G}, \mathbf{Q}} / \mathfrak{m}_{\mathbf{G}, \mathbf{Q}}^{p}$. A boot-strapping argument as in [14] furnishes an isomorphism of the completed local rings themselves, even in the arithmetic setting.

To study the singularities and uniformization of the completed local rings, we may reduce, by considering each $\beta \in \mathbb{B}$ individually, to the case of the Grassmann variety parameterizing two one dimensional subspaces $W_{1}, W_{2}$ of $k^{2}$ that are compatible: $M W_{1} \subseteq W_{2}, M W_{2} \subseteq W_{1}$. (We have simplified the notation from $\left(W_{1}\right)_{\beta}$ to $W_{1}$, etc.) Fix then such a pair $\left(W_{1}, W_{2}\right)$. If $W_{1} \neq \operatorname{Ker}(M)$ then $W_{2}=M W_{1}=\operatorname{Ker}(M)$, and the same holds for any deformation of $W_{1}$ and so $W_{2}$ is constant, being $\operatorname{Ker}(M)$. In this case, we see that the local deformation ring is $k \llbracket x \rrbracket$, where the choice of letter $x$ indicates that it is $W_{1}$ that is being deformed. If $W_{1}=\operatorname{Ker}(M)$ and $W_{2} \neq \operatorname{Ker}(M)$ then the situation is similar and we see that the local deformation ring is $k \llbracket y \rrbracket$, where the choice of letter $y$ indicates that it is $W_{2}$ that is being deformed. Finally, suppose both $W_{1}=\operatorname{Ker}(M)$ and $W_{2}=\operatorname{Ker}(M)$. The subspace $W_{i}$ is spanned by $(1,0)$ and a deformation of it to a local artinian $k$-algebra $D$ is uniquely described by a basis vector $\left(1, d_{i}\right)$ where $d_{i} \in \mathfrak{m}_{D}$. The condition that the deformations are compatible under $f$ is precisely $d_{1} d_{2}=0$ and so we see that the local deformation ring is $k \llbracket x, y \rrbracket /(x y)$. 
Returning to the situation of abelian varieties $(f: A \rightarrow B)$, corresponding to a point $\bar{Q}$, the pair $\left(W_{1}, W_{2}\right)$ is $\left(H^{0}\left(A, \Omega_{A / k}^{1}\right)_{\beta}, H^{0}\left(B, \Omega_{B / k}^{1}\right)_{\beta}\right)=\left(W_{A, \beta}, W_{B, \beta}\right)$ for $\beta \in \mathbb{B}$, and the condition $W_{A, \beta}=\operatorname{Ker}\left(\left(f^{t}\right)^{*}\right)_{\beta}$ is the condition $\beta \in \eta(\bar{Q})$, while the condition $W_{B, \beta}=\operatorname{Ker}\left(f^{*}\right)_{\beta}$ is the condition that $\operatorname{Lie}(f)_{\beta}=0$, namely, $\sigma \circ \beta \in \varphi(\bar{Q})$. Our discussion, therefore, gives immediately the following result.

Theorem 2.4.1. Let $(\underline{A}, f)$ correspond to a point $\bar{Q}$ of $\bar{Y}$, defined over a field $k \supseteq \kappa$. Let $\varphi=\varphi(\underline{A}, f), \eta=\eta(\underline{A}, f)$ and $I=I(\underline{A}, f)=\ell(\varphi) \cap \eta$, then

$$
\widehat{\mathcal{O}}_{\bar{Y}, \bar{Q}} \cong k \llbracket\left\{x_{\beta}: \beta \in \ell(\varphi)\right\},\left\{y_{\beta}: \beta \in \eta\right\} \rrbracket /\left(\left\{x_{\beta} y_{\beta}: \beta \in I\right\}\right) .
$$

This is basically Stamm's theorem, only that Stamm collects together the variables in the following fashion and works over $W(k)$ (which is an easy extension of the argument above).

Theorem 2.4.1 (bis). Let $(\underline{A}, f)$ correspond to a point $\bar{Q}$ of $\bar{Y}$, defined over a field $k \supseteq \kappa$. Let $I=I(\underline{A}, f)$ then

$$
\widehat{\mathcal{O}}_{\bar{Y}, \bar{Q}} \cong k \llbracket\left\{x_{\beta}, y_{\beta}: \beta \in I\right\},\left\{z_{\beta}: \beta \in I^{c}\right\} \rrbracket /\left(\left\{x_{\beta} y_{\beta}: \beta \in I\right\}\right) .
$$

The isomorphism lifts to an isomorphism

$$
\widehat{\mathcal{O}}_{Y, \bar{Q}} \cong W(k) \llbracket\left\{x_{\beta}, y_{\beta}: \beta \in I\right\},\left\{z_{\beta}: \beta \in I^{c}\right\} \rrbracket /\left(\left\{x_{\beta} y_{\beta}-p: \beta \in I\right\}\right) .
$$

The following lemma gives information about a certain stratification of $\bar{Y}$ that is the precursor to the stratification $\left\{W_{\varphi, \eta}\right\}$ studied extensively in this paper.

Lemma 2.4.2. Given $\varphi \subseteq \mathbb{B}$ (respectively, $\eta \subseteq \mathbb{B}$ ) there is a locally closed subset $U_{\phi}$, and a closed subset $U_{\phi}^{+}$(resp. $V_{\eta}$ and $V_{\eta}^{+}$) of $\bar{Y}$ such that $U_{\phi}$ consists of the closed points $\bar{Q}$ with $\varphi(\bar{Q})=\varphi$, and $U_{\phi}^{+}$consists of the closed point $\bar{Q}$ with $\varphi(\bar{Q}) \supseteq \varphi$ (resp., the points $\bar{Q}$ such that $\eta(\bar{Q})=\eta$ and $\eta(\bar{Q}) \supseteq \eta)$.

Furthermore, if $\bar{Q} \in U_{\beta}^{+}$, then $U_{\beta}^{+} \cap \operatorname{Spf}\left(\widehat{\mathcal{O}}_{\bar{Y}}, \bar{Q}\right)$ is equal to $\operatorname{Spf}\left(\widehat{\mathcal{O}}_{\bar{Y}, \bar{Q}}\right)$ if $\beta \notin r(I)$, and is otherwise given by the vanishing of $y_{\sigma^{-1} \circ \beta}$. Similarly, if $\bar{Q} \in V_{\beta}^{+}$, then $V_{\beta}^{+} \cap \operatorname{Spf}\left(\widehat{\mathcal{O}}_{\bar{Y}, \bar{Q}}\right)$ is equal to $\operatorname{Spf}\left(\widehat{\mathcal{O}}_{\bar{Y}}, \bar{Q}\right)$ if $\beta \notin I$, and is otherwise given by the vanishing of $x_{\beta}$.

Proof. It suffices to prove that the $U_{\phi}^{+}$(resp. $V_{\eta}^{+}$) are closed, because $U_{\phi}=U_{\phi}^{+}-\bigcup_{\varphi^{\prime} \supseteq \varphi} U_{\varphi^{\prime}}^{+}$ (resp., $V_{\eta}=V_{\eta}^{+}-\bigcup_{\eta^{\prime} \supsetneq \eta} V_{\eta^{\prime}}^{+}$). Furthermore, since $U_{\phi}^{+}=\bigcap_{\beta \in \varphi} U_{\{\beta\}}^{+}$, we reduce to the case where $\varphi=\{\beta\}$ is a singleton (and similarly for $V_{\eta}^{+}$). From this point we only discuss the case of $U_{\{\beta\}}^{+}$, as it is clear that the same arguments will work for $V_{\{\beta\}}^{+}$.

Recall that $\bar{Q}$, corresponding to $(f: \underline{A} \rightarrow \underline{B})$, satisfies $\varphi(\bar{Q}) \supseteq\{\beta\}$, if and only if $\operatorname{Lie}(f)_{\sigma^{-1} \circ \beta}=0$. Over $\bar{Y}, \operatorname{Lie}\left(\underline{A}^{\text {univ }}\right)_{\gamma}$ and $\operatorname{Lie}\left(\underline{B}^{\text {univ }}\right)_{\gamma}, \gamma \in \mathbb{B}$, are line bundles, and

$$
\operatorname{Lie}(f)_{\gamma}: \operatorname{Lie}\left(\underline{A}^{\text {univ }}\right)_{\gamma} \longrightarrow \operatorname{Lie}\left(\underline{B}^{\text {univ }}\right)_{\gamma}
$$

is a morphism of line bundles and consequently its degeneracy locus $\left\{\operatorname{Lie}(f)_{\gamma}=0\right\}$ is closed.

Moreover, it follows directly from the above description of the variables $x_{\beta}, y_{\beta}$, and the paragraph before Theorem 2.4.1 that if $\bar{Q} \in U_{\beta}^{+}$and $\beta \in r(I)$, then $U_{\beta}^{+} \cap \operatorname{Spf}\left(\widehat{\mathcal{O}}_{\bar{Y}}, \bar{Q}\right)$ is given by the 
vanishing of $y_{\sigma^{-1} \circ \beta}$. Assume now that $\bar{Q} \in U_{\{\beta\}}^{+}$and $\beta \notin r(I(\bar{Q}))$. Since $\beta \in \varphi(\bar{Q})$, we have $\beta \notin r(\eta(\bar{Q}))$. We show that

$$
U=\bigcup_{(\varphi, \eta) \leq(\varphi(\bar{Q}), \eta(\bar{Q}))} U_{\varphi} \cap V_{\eta}
$$

is a Zariski open subset of $\bar{Y}$ which contains $\bar{Q}$ and lies entirely inside $U_{\{\beta\}}^{+}$. First note that if $\left(\varphi\left(\bar{Q}^{\prime}\right), \eta\left(\bar{Q}^{\prime}\right)\right) \leq(\varphi(\bar{Q}), \eta(\bar{Q}))$, then $\beta \in r\left(\eta(\bar{Q})^{c}\right) \subseteq r\left(\eta\left(\bar{Q}^{\prime}\right)^{c}\right) \subseteq \varphi\left(\bar{Q}^{\prime}\right)$, proving that $U \subseteq U_{\{\beta\}}^{+}$. Secondly, it is clear that $\bar{Q} \in U$. Finally, $U$ is a Zariski open subset of $\bar{Y}$, as we have

$$
\bar{Y}-U=\bigcup_{\bar{Q} \notin U_{\varphi}^{+} \cap V_{\eta}^{+}} U_{\varphi}^{+} \cap V_{\eta}^{+}
$$

from definitions.

\subsection{Stratification of $\bar{Y}$.}

Proposition 2.5.1. For an admissible pair $(\varphi, \eta)$ there is a locally closed subset $W_{\varphi, \eta}$ of $\bar{Y}$ with the following property: A closed point $\bar{Q}$ of $\bar{Y}$ has invariants $(\varphi, \eta)$ if and only if $\bar{Q} \in W_{\varphi, \eta}$. Moreover, the subset

$$
Z_{\varphi, \eta}=\bigcup_{\left(\varphi^{\prime}, \eta^{\prime}\right) \geq(\varphi, \eta)} W_{\left(\varphi^{\prime}, \eta^{\prime}\right)}
$$

is closed.

Proof. The proposition follows from Lemma 2.4.2 as we can define

$$
W_{\varphi, \eta}=U_{\phi} \cap V_{\eta}, \quad Z_{\varphi, \eta}=U_{\phi}^{+} \cap V_{\eta}^{+} .
$$

Let $\tau \subseteq \mathbb{B}$. Recall the stratification on $\bar{X}$ introduced in [21, 18]. There is a locally closed subset $W_{\tau}$ of $\bar{X}$ with the property that a closed point $\bar{P}$ of $\bar{X}$ corresponding to $\underline{A}$ belongs to $W_{\tau}$ if and only if $\tau(\underline{A})=\tau$. There is a closed subset $Z_{\tau}$ of $\bar{X}$ with the property that a closed point $\bar{P}$ of $\bar{X}$ corresponding to $\underline{A}$ belongs to $Z_{\tau}$ if and only if $\tau(\underline{A}) \supseteq \tau$. The main properties of these sets are the following:

(1) The collection $\left\{W_{\tau}: \tau \subseteq \mathbb{B}\right\}$ is a stratification of $\bar{X}$ and $\bar{W}_{\tau}=Z_{\tau}=\bigcup_{\tau^{\prime} \supseteq \tau} W_{\tau^{\prime}}$.

(2) Each $W_{\tau}$ is non-empty, regular, quasi-affine and equi-dimensional of dimension $g-\sharp \tau$.

(3) The strata $\left\{W_{\tau}\right\}$ intersect transversally. In fact, let $\bar{P}$ be a closed $k$-rational point of $\bar{X}$. There is a choice of isomorphism

$$
\widehat{\mathcal{O}}_{X, \bar{P}} \cong W(k) \llbracket t_{\beta}: \beta \in \mathbb{B} \rrbracket,
$$

inducing

$$
\widehat{\mathcal{O}}_{\bar{X}, \bar{P}} \cong k \llbracket t_{\beta}: \beta \in \mathbb{B} \rrbracket,
$$

such that for $\tau^{\prime} \subseteq \tau(\bar{P}), W_{\tau^{\prime}}$ (and $\left.Z_{\tau^{\prime}}\right)$ are given in $\operatorname{Spf}\left(\widehat{\mathcal{O}}_{\bar{X}}, \bar{P}\right)$ be the equations $\left\{t_{\beta}=0\right.$ : $\left.\beta \in \tau^{\prime}\right\}$. 
Let $\epsilon: \underline{A}^{\text {univ }} \rightarrow \bar{X}$ be the universal object. The Hodge bundle $\mathcal{L}=\epsilon_{*} \Omega_{\underline{A}^{\text {univ }} / \bar{X}}^{1}$ is a locally free sheaf of $\mathcal{O}_{L} \otimes \mathcal{O}_{\bar{X}}$-modules and so decomposes in line bundles $\mathcal{L}_{\beta}, \mathcal{L}=\oplus_{\beta} \mathcal{L}_{\beta}$. Let $h_{\beta}$ be the partial Hasse invariant, which is a Hilbert modular form of weight $p \sigma^{-1} \circ \beta-\beta$, i.e., a global section of the line bundle $\mathcal{L}_{\sigma^{-1} \circ \beta}^{p} \otimes \mathcal{L}_{\beta}^{-1}$, as in [18]. Then, the divisor of $h_{\beta}$ is reduced and equal to $Z_{\{\beta\}}$. For every closed point $\bar{P} \in Z_{\{\beta\}}$, one can trivialize the line bundle $\mathcal{L}_{\sigma^{-1} \circ \beta}^{p} \otimes \mathcal{L}_{\beta}^{-1}$ over $\operatorname{Spf}\left(\widehat{\mathcal{O}}_{\bar{X}}, \bar{P}\right)$ and thus view $h_{\beta}$ as an element of $\widehat{\mathcal{O}}_{\bar{X}}, \bar{P}$. The variable $t_{\beta}$ can be chosen to coincide with that function $h_{\beta}$. We now prove the fundamental properties of the stratification of $\bar{Y}$.

Theorem 2.5.2. Let $(\varphi, \eta)$ be an admissible pair, $I=\ell(\varphi) \cap \eta$.

(1) $W_{\varphi, \eta}$ is non-empty, and its Zariski closure is $Z_{\varphi, \eta}$. The collection $\left\{W_{\varphi, \eta}\right\}$ is a stratification of $\bar{Y}$ by $3^{g}$ strata.

(2) $W_{\varphi, \eta}$ and $Z_{\varphi, \eta}$ are equi-dimensional, and

$$
\operatorname{dim}\left(W_{\varphi, \eta}\right)=\operatorname{dim}\left(Z_{\varphi, \eta}\right)=2 g-(\sharp \varphi+\sharp \eta) .
$$

(3) The irreducible components of $\bar{Y}$ are the irreducible components of the strata $Z_{\varphi, \ell\left(\varphi^{c}\right)}$ for $\varphi \subseteq \mathbb{B}$.

(4) Let $\bar{Q}$ be a closed point of $\bar{Y}$ with invariants $(\varphi, \eta), I=\ell(\varphi) \cap \eta$. For an admissible pair $\left(\varphi^{\prime}, \eta^{\prime}\right)$, we have $\bar{Q} \in Z_{\varphi^{\prime}, \eta^{\prime}}$ if and only if we have:

$$
\varphi \supseteq \varphi^{\prime} \supseteq \varphi-r(I), \quad \eta \supseteq \eta^{\prime} \supseteq \eta-I .
$$

In that case, write $\varphi^{\prime}=\varphi-J, \eta^{\prime}=\eta-K \quad$ (so that $\ell(J) \subseteq I, K \subseteq I$ and $\left.\ell(J) \cap K=\emptyset\right)$. We have:

$$
\widehat{\mathcal{O}}_{Z_{\varphi^{\prime}, \eta^{\prime}}, \bar{Q}}=\widehat{\mathcal{O}}_{\bar{Y}, \bar{Q}} / \mathcal{I}
$$

where $\mathcal{I}$ is the ideal

$$
\mathcal{I}=\left\langle\left\{x_{\beta}: \beta \in I-K\right\},\left\{y_{\gamma}: \gamma \in I-\ell(J)\right\}\right\rangle .
$$

This implies that each stratum in the stratification $\left\{Z_{\varphi, \eta}\right\}$ is non-singular.

Proof. We begin with the proof of assertion (4), keeping the notation $\varphi=\varphi(\bar{Q}), \eta=\eta(\bar{Q}), I=$ $I(\bar{Q})$. We need the following fact.

Claim: There exists a Zariski open set $U$ with $\bar{Q} \in U$, such that for every closed point $\bar{Q}^{\prime} \in U$ one has

$$
\varphi\left(\bar{Q}^{\prime}\right) \supseteq \varphi-r(I), \quad \eta\left(\bar{Q}^{\prime}\right) \supseteq \eta-I .
$$

In words, locally Zariski, $(\varphi, \eta)$ can become smaller only at $\beta \in I$.

To prove the claim, choose

$$
U=\bar{Y}-\left[\bigcup_{\beta \in r(\eta-I)} U_{\beta}^{+} \cup \bigcup_{\beta \in \ell(\varphi)-I} V_{\beta}^{+}\right] .
$$

We verify that this choice of $U$ is adequate. Firstly, $\bar{Q} \in U$. Indeed, since $\varphi=r\left(\eta^{c}\right) \cup r(I)$, if $\beta \in r(\eta)-r(I)$ then $\beta \notin \varphi$. Similarly, since $\eta=\ell\left(\varphi^{c}\right) \cup I$, if $\beta \in \ell(\varphi)-I$ then $\beta \notin \eta$. That shows that $\bar{Q} \notin \bigcup_{\beta \in r(\eta-I)} U_{\beta}^{+} \cup \bigcup_{\beta \in \ell(\varphi)-I} V_{\beta}^{+}$and so that $\bar{Q} \in U$. 
Let $\bar{Q}^{\prime} \in U$ then: (i) Since $\varphi\left(\bar{Q}^{\prime}\right) \subseteq r(\eta-I)^{c}$ we have $\ell\left(\varphi\left(\bar{Q}^{\prime}\right)^{c}\right) \supseteq \eta-I$ and by admissibility $\eta\left(\bar{Q}^{\prime}\right) \supseteq \eta-I$; (ii) $\eta\left(\bar{Q}^{\prime}\right) \subseteq(\ell(\varphi)-I)^{c}$ and so $r\left(\eta\left(\bar{Q}^{\prime}\right)^{c}\right) \supseteq \varphi-r(I)$ and admissibility gives $\varphi\left(\bar{Q}^{\prime}\right) \supseteq \varphi-r(I)$. Thus, the set $U$ is contained in $U_{\varphi-r(I)}^{+} \cap V_{\eta-I}^{+}$, and our claim is proved.

By Lemma 2.4.2, for the ideal $\mathcal{I}$ in the theorem,

$$
\begin{aligned}
V(\mathcal{I}) & =\bigcap_{\gamma \in I-\ell(J)} U_{\sigma \circ \gamma}^{+} \cap \bigcap_{\beta \in I-K} V_{\beta}^{+} \cap \operatorname{Spf}\left(\widehat{\mathcal{O}}_{\bar{Y}, \bar{Q}}\right) \\
& =\left(\bigcap_{\gamma \in I-\ell(J)} U_{\sigma \circ \gamma}^{+} \cap U_{\varphi-r(I)}^{+}\right) \cap\left(\bigcap_{\beta \in I-K} V_{\beta}^{+} \cap V_{\eta-I}^{+}\right) \cap \operatorname{Spf}\left(\widehat{\mathcal{O}}_{\bar{Y}, \bar{Q}}\right) \\
& =Z_{\varphi-J, \eta-K} .
\end{aligned}
$$

(We made use of the Claim in the second equality.) This concludes the proof of assertion (4).

There is a point $\bar{Q}$ with invariants $(\varphi, \eta)=(\mathbb{B}, \mathbb{B})$ - it corresponds to $(\underline{A}, H)$, where $\underline{A}$ is superspecial and $H$ is the kernel of Frobenius. The point $\bar{Q}$ belongs to every strata $Z_{\varphi, \eta}$ and hence each $Z_{\varphi, \eta}$ is non-empty. The above computations also show that $Z_{\varphi, \eta}$ is pure dimensional and $\operatorname{dim}\left(Z_{\varphi, \eta}\right)=2 g-(\sharp \varphi+\sharp \eta)$.

Since $Z_{\varphi, \eta}-W_{\varphi, \eta}=\bigcup_{\left(\varphi^{\prime}, \eta^{\prime}\right) \supsetneqq(\varphi, \eta)} Z_{\left(\varphi^{\prime}, \eta^{\prime}\right)}$ is a union of lower-dimensional strata, it follows that $W_{\varphi, \eta}$ is non-empty for all admissible $(\varphi, \eta)$. The computations above show that $W_{\varphi, \eta}$ is pure-dimensional and $\operatorname{dim}\left(W_{\varphi, \eta}\right)=2 g-(\sharp \varphi+\sharp \eta)$ as well.

We know that $Z_{\varphi, \eta}$ is closed and contains $W_{\varphi, \eta}$, hence $\bar{W}_{\varphi, \eta}$. Dimension considerations imply that $\bar{W}_{\varphi, \eta}$ must be a union of irreducible components of $Z_{\varphi, \eta}$. If $\bar{W}_{\varphi, \eta} \neq Z_{\varphi, \eta}$, then the remaining components of $Z_{\varphi, \eta}$ are contained in $\bigcup_{\left(\varphi^{\prime}, \eta^{\prime}\right) \supsetneqq(\varphi, \eta)} Z_{\left(\varphi^{\prime}, \eta^{\prime}\right)}$, which is not possible by dimension considerations.

It remains only to prove assertion (3). First note that, by admissibility, $\operatorname{dim}\left(Z_{\varphi, \eta}\right)=g$ exactly when $\eta=\ell(\varphi)^{c}$. Let $C$ be an irreducible component of $\bar{Y}$. Since $C$ is contained in the union of all $g$-dimensional closed strata, it must be contained in a single one, i.e., $C \subseteq Z_{\varphi, \eta}$ for some $(\varphi, \eta)$. Therefore, $C$ must be an irreducible component of $Z_{\varphi, \eta}$. Conversely, every irreducible component of $Z_{\varphi, \ell(\varphi)^{c}}$ is $g$-dimensional, and hence an irreducible component of $\bar{Y}$. In particular, $\bar{Y}$ is of pure dimension $g$.

Corollary 2.5.3. The singular locus $\bar{Y}^{\text {sing }}$ of $\bar{Y}$ has the following description.

$$
\bar{Y}^{\text {sing }}=\bar{Y}-\bigcup_{\varphi \subset \mathbb{B}} W_{\varphi, \ell\left(\varphi^{c}\right)}=\bigcup_{\substack{(\varphi, \eta) \\ \ell(\varphi) \cap \eta \neq \emptyset}} W_{\varphi, \eta} .
$$

If $\bar{Q} \in \bar{Y}^{\text {sing }}$ then there are $2^{\sharp I(\bar{Q})}$ irreducible components passing through $\bar{Q}$, all g-dimensional.

Definition 2.5.4. Recall that $\mathbb{B}_{\mathfrak{p}}=\left\{\beta \in \mathbb{B}: \beta^{-1}(p W(\kappa))=\mathfrak{p}\right\}$. Let $\mathfrak{t}$ be an ideal of $\mathcal{O}_{L}$ dividing $p$. Let $\mathfrak{t}^{*}=p / \mathfrak{t}\left(\right.$ so $\left.\mathfrak{t t}^{*}=p \mathcal{O}_{L}\right)$. Let $\mathbb{B}_{\mathfrak{t}}=\cup_{\mathfrak{p} \mid \mathfrak{t}} \mathbb{B}_{\mathfrak{p}}$, and let $f(\mathfrak{t})=\sum_{\mathfrak{p} \mid \mathfrak{t}} f(\mathfrak{p} / p)$ be the sum of the residue degrees. 
The following proposition is clear from definitions.

Proposition 2.5.5. We have

(1) $\bar{Y}_{F}=Z_{\mathbb{B}, \emptyset}$, and $\bar{Y}_{V}=Z_{\emptyset, \mathbb{B}}$.

(2) $\bar{X}^{\text {ord }}=W_{\emptyset}$.

(3) $\bar{Y}^{\text {ord }}=\cup_{\mathfrak{t} \mid p} W_{\mathbb{B}_{\mathfrak{t}}, \mathbb{B}_{\mathfrak{t}^{*}}}$.

(4) $\bar{Y}_{F}^{\text {ord }}=W_{\mathbb{B}, \emptyset} ; \bar{Y}_{V}^{\text {ord }}=W_{\emptyset, \mathbb{B}}$.

2.6. The fibres of $\pi: \bar{Y} \rightarrow \bar{X}$.

A certain Grassmann variety. Fix a closed $k$-rational point $\bar{P}$ of $\bar{X}$ corresponding to $\underline{A}$, where $k$ is an algebraically closed field. Let $\mathbb{D}=\mathbb{D}(\underline{A}[p])=\oplus_{\beta \in \mathbb{B}} \mathbb{D}_{\beta}$; each $\mathbb{D}_{\beta}$ is a 2-dimensional vector space over $k$ on which $\mathcal{O}_{L}$ acts via $\beta$. Recall from 92.2 that the kernel of Frobenius and the Kernel of Verschiebung, two Dieudoné submodules of $\mathbb{D}$, decompose as

$$
\operatorname{Ker}(\operatorname{Fr})=\oplus_{\beta \in \mathbb{B}} \operatorname{Ker}(\operatorname{Fr})_{\beta}, \quad \operatorname{Ker}(\operatorname{Ver})=\oplus_{\beta \in \mathbb{B}} \operatorname{Ker}(\operatorname{Ver})_{\beta},
$$

where each $\operatorname{Ker}(\operatorname{Fr})_{\beta}, \operatorname{Ker}(\operatorname{Ver})_{\beta}$ is a one dimensional subspace of $\mathbb{D}_{\beta}$. By Lemma 2.3.3, we have

$$
\beta \in \tau(\underline{A}) \Longleftrightarrow \operatorname{Ker}(\operatorname{Fr})_{\beta}=\operatorname{Ker}(\operatorname{Ver})_{\beta} .
$$

Consider the variety $\mathscr{G}=\mathscr{G}(\bar{P})$ parameterizing subspaces $\mathbb{H}=\oplus \mathbb{H}_{\beta}$ of $\mathbb{D}$ satisfying the conditions:

- $\mathbb{H}_{\beta} \subset \mathbb{D}_{\beta}$ is 1-dimensional.

- $\operatorname{Fr}(\mathbb{H}(\beta)) \subseteq \mathbb{H}_{\sigma \circ \beta}$.

- $\operatorname{Ver}(\mathbb{H}(\beta)) \subseteq \mathbb{H}_{\sigma^{-1} \circ \beta}$.

We give $\mathscr{G}$ the scheme structure of a closed reduced subscheme of $\left(\mathbb{P}_{k}^{1}\right)^{g}$. It is a generalized Grassmann variety.

Define a morphism

$$
g: \pi^{-1}(\bar{P})_{\text {red }} \longrightarrow \mathscr{G},
$$

as follows. We use the identification $\mathbb{D}=H_{\mathrm{dR}}^{1}\left(\underline{A}, \mathcal{O}_{\underline{A}}\right)$. The universal family $\left(f: \underline{A}^{\text {univ }} \rightarrow \underline{B}^{\text {univ }}\right)$ over the reduced fibre $\pi^{-1}(\bar{P})_{\text {red }}$ produces a sub-vector bundle of $\mathbb{D} \times \pi^{-1}(\bar{P})_{\text {red }}$ by considering $f^{*} \mathbb{H}_{\mathrm{dR}}^{1}\left(\underline{B}, \mathcal{O}_{\underline{B}}\right)$, which point-wise is $f^{*} \mathbb{H}_{\mathrm{dR}}^{1}\left(\underline{B}_{x}, \mathcal{O}_{\underline{B}_{x}}\right)=\mathbb{D}(f)\left(\mathbb{D}\left(\underline{B}_{x}[p]\right)\right)\left(x \in \pi^{-1}(\bar{P})_{\text {red }}\right)$, and so is a subspace of the kind parameterized by $\mathscr{G}$. By the universal property of Grassmann variety $\left(\mathbb{P}_{k}^{1}\right)^{g}=\operatorname{Grass}(1,2)^{g}$, we get a morphism $g:: \pi^{-1}(\bar{P})_{\text {red }} \rightarrow\left(\mathbb{P}_{k}^{1}\right)^{g}$ that factors through $\mathscr{G}$, because it does so at every closed point of $\pi^{-1}(\bar{P})_{\text {red }}$. We note that for every $x$ as above $\mathbb{D} / f^{*} \mathbb{H}_{\mathrm{dR}}^{1}\left(\underline{B}_{x}, \mathcal{O}_{\underline{B}_{x}}\right)=\mathbb{D}\left(\operatorname{Ker}\left(f_{x}\right)\right)$ and so it is clear that $g$ is injective on geometric points and in fact, by the theory of Dieudonné modules, bijective. We have therefore constructed a bijective morphism

$$
g: \pi^{-1}(\bar{P})_{\text {red }} \longrightarrow \mathscr{G} .
$$

Since $g$ is a morphism between projective varieties, it is closed and hence it is a homeomorphism. We will use this in what follows.

It will be convenient for us to think of the fibre $\pi^{-1}(\bar{P})_{\text {red }}$ entirely in terms of $\mathscr{G}$. To this end, we provide some definitions. For $\mathbb{H} \subset \mathbb{D}$ as above, define

$$
\varphi(\mathbb{H})=\left\{\beta \in \mathbb{B}: \mathbb{H}_{\beta}=\operatorname{Ker}(\operatorname{Ver})_{\beta}\right\},
$$


and

$$
\eta(\mathbb{H})=\left\{\beta \in \mathbb{B}: \mathbb{H}_{\beta}=\operatorname{Ker}(\mathrm{Fr})_{\beta}\right\} .
$$

Lemma 2.6.1. Let $H \subset \underline{A}[p]$ be a subgroup scheme such that $(\underline{A}, H) \in \pi^{-1}(\bar{P})$. Let $f: A \rightarrow A / H$ be the canonical map. Let $\mathbb{H}=\operatorname{Im}[\mathbb{D}(f)]=\operatorname{Ker}[\mathbb{D}(\underline{A}[p]) \rightarrow \mathbb{D}(H)]$. Then,

$$
\varphi(\underline{A}, H)=\varphi(\mathbb{H}), \quad \eta(\underline{A}, H)=\eta(\mathbb{H}) .
$$

Proof. By Lemma 2.3.3, we have

$$
\begin{aligned}
\varphi(\underline{A}, H) & =\left\{\beta \in \mathbb{B}: \operatorname{Im}(\mathbb{D}(f))_{\beta}=\operatorname{Im}\left(\mathbb{D}\left(\operatorname{Fr}_{A}\right)\right)_{\beta}\right\} \\
& =\left\{\beta \in \mathbb{B}: \mathbb{H}_{\beta}=(\operatorname{Im}(\operatorname{Fr}))_{\beta}\right\} \\
& =\left\{\beta \in \mathbb{B}: \mathbb{H}_{\beta}=\operatorname{Ker}(\text { Ver })_{\beta}\right\} \\
& =\varphi(\mathbb{H}) .
\end{aligned}
$$

The argument for $\eta(\underline{A}, H)$ is similar.

We can now study the induced stratification on $\pi^{-1}(\bar{P})_{\text {red }}$ by means of $\mathscr{G}$.

Corollary 2.6.2. $\pi^{-1}(\bar{P})_{\text {red }} \cap W_{\varphi, \eta}$ is homeomorphic to the locally closed subset of $\mathscr{G}$ parameterizing subspaces $\mathbb{H}$ with $\varphi(\mathbb{H})=\varphi, \eta(\mathbb{H})=\eta$. Its dimension is thus at most $g-\sharp(\varphi \cup \eta)$.

Remark 2.6.3. We will see (Corollary 2.6.7) that the equality holds if the fibre is non-empty.

\section{The relation between the stratifications on $\bar{Y}$ and $\bar{X}$.}

Theorem 2.6.4. (1) Let $C$ be a component of $Z_{\varphi, \eta}$; then $\pi(C)$ is a component of $Z_{\varphi \cap \eta}$.

(2) $\pi\left(Z_{\varphi, \eta}\right)=Z_{\varphi \cap \eta}$.

(3) On every component of $Z_{\varphi, \eta}$ (or $W_{\varphi, \eta}$ ) the type is generically $\varphi \cap \eta$.

Remark 2.6.5. The type is not necessarily constant on $W_{\varphi, \eta}$. In fact,

$$
\pi\left(W_{\varphi, \eta}\right)=\bigcup_{\left[\varphi(\underline{A}, H) \Delta \underline{\underline{A}(\underline{A}, H)]^{c} \supseteq \tau^{\prime}}\right.} W_{\tau^{\prime}} \underline{\varphi} \cap
$$

See Proposition 2.6 .16 below.

Proof. For every point $y \in \pi(C), \operatorname{dim}\left(\pi^{-1}(y) \cap C\right) \leq g-\sharp(\varphi \cup \eta)$, by Corollary 2.6.2, Therefore,

$$
\begin{aligned}
\operatorname{dim}(\pi(C)) & \geq \operatorname{dim}(C)-(g-\sharp(\varphi \cup \eta)) \\
& =2 g-(\sharp \varphi+\sharp \eta)-(g-\sharp(\varphi \cup \eta)) \\
& =g-\sharp(\varphi \cap \eta) .
\end{aligned}
$$

On the other hand, since $\tau(y) \supseteq \varphi \cap \eta$, we have $\pi(C) \subseteq Z_{\varphi \cap \eta}$. Moreover, $\operatorname{dim}\left(Z_{\varphi \cap \eta}\right)=g-\sharp(\varphi \cap \eta)$. Since $\pi$ is proper, $\pi(C)$ is closed and irreducible. By comparing the dimensions, we conclude that $\pi(C)$ is an irreducible component of $Z_{\varphi \cap \eta}$. This is part (1) of the theorem.

We now prove part (2) of the theorem. Let $C \subseteq Z_{\varphi \cap \eta}$ be an irreducible component, and let $\bar{P}$ be a closed point of $C$ corresponding to $\underline{A}$. We want to prove that $\pi^{-1}(\bar{P}) \cap Z_{\varphi, \eta} \neq \emptyset$. We provide two proofs for that. 
- A "pure thought" argument. $\pi^{-1}(\bar{P}) \cap Z_{\varphi, \eta}$ depends entirely on $\mathbb{D}(\underline{A}[p])$, which is determined by the type [21, Theorem 3.8]. It is a consequence of part (1) that there are other points $\bar{P}^{\prime}$ of the same type as $\bar{P}$ lying in the image of $Z_{\varphi, \eta}$. Therefore, $\pi^{-1}(\bar{P}) \cap Z_{\varphi, \eta} \neq \emptyset$.

The second method is more instructive and will be used again in the sequel.

- An explicit construction. We construct a Dieudonné submodule $\mathbb{H} \subseteq \mathbb{D}(\underline{A}[p])$ subject to the conditions:

$$
\begin{array}{ll}
\forall \beta \in \varphi: & \mathbb{H}_{\beta}=\operatorname{Ker}(\text { Ver })_{\beta}, \\
\forall \beta \in \eta: & \mathbb{H}_{\beta}=\operatorname{Ker}(\operatorname{Fr})_{\beta} .
\end{array}
$$

Lemma 2.6.6. For any choice of $\mathbb{H}_{\beta}$ for $\beta \in(\varphi \cup \eta)^{c}$, $\mathbb{H}=\oplus_{\beta \in \mathbb{B}} \mathbb{H}_{\beta}$ satisfies the required conditions: namely, it is a Dieudonné submodule, corresponding to a subgroup $H \subset \underline{A}[p]$ with invariants $\left(\varphi^{\prime}, \eta^{\prime}\right) \geq(\varphi, \eta)$.

Proof. (Of Lemma) First note that the formulas $\ell(\varphi) \cup \eta=\varphi \cup r(\eta)=\mathbb{B}$ give

$$
\epsilon \in(\varphi \cup \eta)^{c} \Longrightarrow\left\{\begin{array}{l}
\sigma \circ \epsilon \in \varphi \\
\sigma^{-1} \circ \epsilon \in \eta .
\end{array}\right.
$$

Therefore,

$$
\mathbb{H}_{\sigma \circ \epsilon}=\operatorname{Ker}(\operatorname{Ver})_{\sigma \circ \epsilon}, \quad \mathbb{H}_{\sigma^{-1} \circ \epsilon}=\operatorname{Ker}(\mathrm{Fr})_{\sigma^{-1} \circ \epsilon}
$$

Since we always have $\operatorname{Fr}\left(\mathbb{H}_{\epsilon}\right) \subseteq \operatorname{Ker}(\operatorname{Ver})_{\sigma \circ \epsilon}, \operatorname{Ver}\left(\mathbb{H}_{\epsilon}\right) \subseteq \operatorname{Ker}(\operatorname{Fr})_{\sigma^{-1} \circ \epsilon}$, we conclude that

$$
\operatorname{Fr}\left(\mathbb{H}_{\epsilon}\right) \subseteq \mathbb{H}_{\sigma \circ \epsilon}, \quad \operatorname{Ver}\left(\mathbb{H}_{\epsilon}\right) \subseteq \mathbb{H}_{\sigma^{-1} \circ \epsilon}, \quad \epsilon \in(\varphi \cup \eta)^{c}
$$

Suppose now that $\epsilon \in \varphi$. Then $\mathbb{H}_{\epsilon}=\operatorname{Ker}(\text { Ver })_{\epsilon}$ and so $V\left(\mathbb{H}_{\epsilon}\right)=0 \subset \mathbb{H}_{\sigma^{-1} \circ \epsilon}$. On the other hand, we have that $\operatorname{Fr}\left(\mathbb{H}_{\epsilon}\right) \subseteq \mathbb{H}_{\sigma \circ \epsilon}$ if $\epsilon \in \varphi \cap \eta$ (because $\operatorname{Fr}\left(\mathbb{H}_{\epsilon}\right)=0$ ), or if $\sigma \circ \epsilon \in \varphi$ (because then $\left.\operatorname{Fr}\left(\mathbb{H}_{\epsilon}\right) \subseteq \operatorname{Ker}(\operatorname{Ver})_{\sigma \circ \epsilon}=\mathbb{H}_{\sigma \circ \epsilon}\right)$. Therefore, it is enough to show that we cannot have $\epsilon \in \varphi, \epsilon \notin \varphi \cap \eta$, and $\sigma \circ \epsilon \notin \varphi$. This follows readily from $\ell\left(\varphi^{c}\right) \subseteq \eta$.

The argument for $\epsilon \in \eta$ is entirely similar and hence omitted. The claim concerning the invariants is clear from the construction.

The existence of a bijective morphism $g: \pi^{-1}(\bar{P})_{\text {red }} \longrightarrow \mathscr{G}$, implies that $\pi^{-1}(\bar{P}) \cap Z_{\varphi, \eta} \neq \emptyset$, and hence part (2) follows. Finally, part (3) follows immediately from part (1).

We remark that since $g$ is a homeomorphism, the second method of the proof gives the following interesting result:

Corollary 2.6.7. Let $\bar{P}$ be a closed point of $\bar{X}$ such that $\tau(\bar{P}) \supseteq \varphi \cap \eta$. Then,

$$
\operatorname{dim}\left(\pi^{-1}(\bar{P}) \cap Z_{\varphi, \eta}\right)=\operatorname{dim}\left(\pi^{-1}(\bar{P}) \cap W_{\varphi, \eta}\right)=g-\sharp(\varphi \cup \eta) .
$$

Corollary 2.6.8. On every irreducible component of $Z_{\varphi, \eta}$ there is a point $\bar{Q}$ such that $\pi(\bar{Q})$ is superspecial.

Proof. Let $C \subseteq Z_{\varphi, \eta}$ be an irreducible component. Then $\pi(C)$ is an irreducible component of $Z_{\varphi \cap \eta}$, and hence contains a superspecial point; in the case $p$ is inert this follows from $W_{\tau}$ being quasi-affine for $\tau \neq \mathbb{B}$ [21, Proposition 2.19]. In the general case, see [18, §1]. In both cases note that $W_{\tau}$ is denoted there $W_{\tau}^{0}$ and $Z_{\tau}$ is denoted there $W_{\tau}$. 
Definition 2.6.9. A closed stratum $Z_{\varphi, \eta}$ is called horizontal if $\pi: Z_{\varphi, \eta} \rightarrow \bar{X}$ is finite and surjective (equivalently, dominant and quasi-finite).

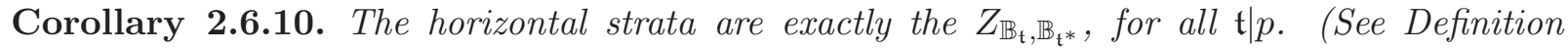
2.5.4.

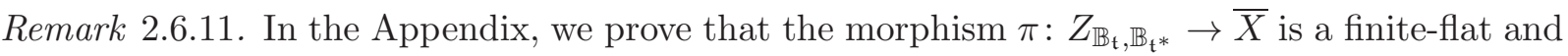
purely inseparable morphism of degree $p^{f(\mathfrak{t})}$.

Our next goal is a detailed study of the fibre $\pi^{-1}(\bar{P})_{\text {red, }}$, where $\bar{P}$ is a superspecial point; it is used in the proof of Theorem 2.6 .13 below.

Let $\bar{P}$ be a superspecial point of $\bar{X}$, corresponding to $\underline{A}$ defined over a perfect field $k$. Let $\mathbb{D}=\mathbb{D}(\underline{A}[p])$. Let $S \subset \mathbb{B}$ be a spaced subset: that is, $\beta \in S \Rightarrow \sigma \circ \beta \notin S$. We define

$$
\mathcal{F}_{S} \subseteq \pi^{-1}(\bar{P})_{\text {red }},
$$

to be the closed subset of $\pi^{-1}(\bar{P})_{\text {red }}$ whose geometric points are $\bar{Q}=(\underline{A}, H)$ such that, letting $\mathbb{H}=\operatorname{Ker}[\mathbb{D}(\underline{A}[p]) \rightarrow \mathbb{D}(H)]=\mathbb{D}(\underline{A}[p] / H)$,

$$
\mathbb{H}_{\beta}=\operatorname{Ker}(\operatorname{Fr})_{\beta}\left(=\operatorname{Ker}(\operatorname{Ver})_{\beta}\right), \quad \forall \beta \notin S .
$$

Let $\mathcal{F}_{S}^{\circ}$ denote the open subset of $\mathcal{F}_{S}$ where $\mathbb{H}_{\beta} \neq \operatorname{Ker}(\operatorname{Fr})_{\beta}, \forall \beta \in S$.

Lemma 2.6.12. Let $S \subset \mathbb{B}$ be a spaced subset.

(1) $\mathcal{F}_{S}$ and $\mathcal{F}_{S}^{\circ}$ are irreducible of dimension $\sharp S$. In fact, there are geometrically bijective, finite morphisms $\mathcal{F}_{S} \rightarrow\left(\mathbb{P}_{k}^{1}\right)^{\sharp S}$ and $\mathcal{F}_{S}^{\circ} \rightarrow\left(\mathbb{A}_{k}^{1}\right)^{\sharp S}$.

(2) $\mathcal{F}_{S_{1}} \cap \mathcal{F}_{S_{2}}=\mathcal{F}_{S_{1} \cap S_{2}}$.

(3) The collection of locally closed sets $\left\{\mathcal{F}_{S}^{\circ}: S \subseteq \mathbb{B}\right.$ spaced $\}$ forms a stratification of $\pi^{-1}(\bar{P})_{\text {red }}$ by irreducible locally closed subsets. In fact,

$$
\mathcal{F}_{S}^{\circ}=W_{S^{c}, S^{c}} \cap \pi^{-1}(\bar{P})_{\text {red }} .
$$

Proof. The proof is essentially a series of simple observations. First, $\mathcal{F}_{S}$ is homeomorphic via $g: \pi^{-1}(\bar{P})_{\text {red }} \longrightarrow \mathscr{G}$ to the closed subset of $\mathscr{G}(\bar{P})$ where $\mathbb{H}_{\beta}=\operatorname{Ker}(\operatorname{Fr})_{\beta}=\operatorname{Ker}(\operatorname{Ver})_{\beta}$ for all $\beta \notin S$, and no conditions are imposed at $\beta \in S$ (cf. the proof of Lemma 2.6.6). This subset of $\mathscr{G}$, viewed with the reduced induced structure, is clearly isomorphic to $\left(\mathbb{P}_{k}^{1}\right)^{\sharp S}$. Similarly, $\mathcal{F}_{S}^{\circ}$ is mapped via $g$ to a closed subscheme of $\mathscr{G}$ isomorphic to $\left(\mathbb{A}_{k}^{1}\right)^{\sharp S}$.

The second part of the lemma is immediate from the definitions (the intersection is considered set-theoretically).

As for the last part, first note that $\overline{\mathcal{F}_{S}^{\circ}}=\mathcal{F}_{S}$; indeed, considered on $\mathscr{G}$ this just says that $\left(\mathbb{A}_{k}^{1}\right)^{\sharp S}$ in dense in $\left(\mathbb{P}_{k}^{1}\right)^{\sharp S}$. Next, from the definitions we have

$$
\mathcal{F}_{S}=\coprod_{T \subseteq S} \mathcal{F}_{T}^{\circ},
$$

and

$$
\mathcal{F}_{S}^{\circ}=W_{S^{c}, S^{c}} \cap \pi^{-1}(\bar{P})_{\text {red }} .
$$

Theorem 2.6.13. Let $C$ be an irreducible component of $Z_{\varphi, \eta}$. Then

$$
C \cap \bar{Y}_{F} \cap \bar{Y}_{V} \neq \emptyset \text {. }
$$


Remark 2.6.14. Note that $\bar{Y}_{F} \cap \bar{Y}_{V}$ consists of points $(\underline{A}, H)$, where $H$ is both the kernel of Frobenius and the kernel of Verschiebung. Such an abelian variety $A$ is superspecial, and the points $\bar{Y}_{F} \cap \bar{Y}_{V}$ are thus the finitely many points $\left(\operatorname{Fr}_{A}: \underline{A} \rightarrow \underline{A}^{(p)}\right)$, where $\underline{A}$ ranges over the finitely many superspecial points in $\bar{X}$. Those finitely many points "hold together" all the components of $\bar{Y}$.

Proof. Let $S=(\varphi \cup \eta)^{c}$. Then $S$ is spaced: If $\beta \in S$ then $\beta \in \varphi^{c} \cap \eta^{c}$ and so $\sigma \circ \beta \in r\left(\eta^{c}\right) \subseteq \varphi$ and so $\sigma \circ \beta \notin \varphi^{c}$, hence $\sigma \circ \beta \notin S$.

Let $\bar{Q} \in C$ be a closed point such that $\bar{P}=\pi(\bar{Q})$ is superspecial (Corollary 2.6.8). Consider $\mathcal{F}_{S}^{\circ} \subseteq \pi^{-1}(\bar{P})$. Recall from Lemma 2.6.12 that $\mathcal{F}_{S}^{\circ}=W_{S^{c}, S^{c}} \cap \pi^{-1}(\bar{P})$, and we have $\varphi \subseteq S^{c}, \eta \subseteq S^{c}$. Any $x \in \mathcal{F}_{S}^{\circ}$ belongs to $Z_{\varphi, \eta}$, and since $Z_{\varphi, \eta}$ is nonsingular (Theorem 2.5.2), there exists a unique component of $Z_{\varphi, \eta}$ passing through $x$; we call that component $C_{x}$. We distinguish two cases.

Case 1: $\operatorname{dim}\left(\mathcal{F}_{S}^{\circ}\right) \geq 1$. This case is equivalent to $\varphi \cup \eta \neq \mathbb{B}$, by Lemma 2.6.12. By assumption, $\mathcal{F}_{S}^{\circ}$ has infinitely many points, while $Z_{\varphi, \eta}$ has only finitely many components. Therefore, there exists a component $C^{\prime}$ of $Z_{\varphi, \eta}$ such that $C^{\prime} \cap \mathcal{F}_{S}^{\circ}$ is dense in $\mathcal{F}_{S}^{\circ}$ and, thus, $C^{\prime} \supseteq \mathcal{F}_{S}$. But then, since $Z_{\varphi, \eta}$ is nonsingular, no other component of $Z_{\varphi, \eta}$ intersects $\mathcal{F}_{S}$.

By assumption $\bar{Q} \in Z_{\varphi, \eta}$ is so that $\pi(\bar{Q})$ is superspecial. Hence, by Lemma 2.3.3, we have $\varphi(\bar{Q})=\eta(\bar{Q})$. Now, $(\varphi(\bar{Q}), \eta(\bar{Q})) \geq(\varphi, \eta)$ gives that $\varphi(\bar{Q}) \supseteq \varphi \cup \eta=S^{c}$ and $\eta(\bar{Q}) \supseteq \varphi \cup \eta=S^{c}$. Because

$$
\mathcal{F}_{S}=\left\{x \in \pi^{-1}(\bar{P}):(\varphi(x), \eta(x)) \geq\left(S^{c}, S^{c}\right)\right\},
$$

we conclude that $\bar{Q} \in \mathcal{F}_{S}$. It follows that $C^{\prime}=C$, and since $\mathcal{F}_{S} \cap \bar{Y}_{F} \cap \bar{Y}_{V} \neq \emptyset$, our proof is complete in this case.

Case 2: $\operatorname{dim}\left(\mathcal{F}_{S}^{\circ}\right)=0$. This case is equivalent to $\varphi \cup \eta=\mathbb{B}$, and so $S=\emptyset$. In this case $\mathcal{F}_{S}=\mathcal{F}_{S}^{\circ}=\bar{Y}_{F} \cap \bar{Y}_{V} \cap \pi^{-1}(\bar{P})$. As above, $\bar{Q} \in \mathcal{F}_{S}$ and so $\bar{Q} \in \bar{Y}_{F} \cap \bar{Y}_{V} \cap \pi^{-1}(\bar{P})$.

This complete the proof.

The proof reveals an interesting fact:

Corollary 2.6.15. Let $(\varphi, \eta)$ be an admissible pair, and $S=(\varphi \cup \eta)^{c}$. Let $\bar{P}$ be a superspecial point. There is a unique component $C$ of $Z_{\varphi, \eta}$ that intersects $\pi^{-1}(\bar{P})$. We have $C \cap \pi^{-1}(\bar{P})=\mathcal{F}_{S}$. Moreover, any superspecial point $\bar{P}$ belongs to $\pi\left(Z_{\varphi, \eta}\right)$.

We end this section by refining our knowledge on the relationship between the strata on $\bar{Y}$ and on $\bar{X}$.

Proposition 2.6.16. Let $(\varphi, \eta)$ be an admissible pair. Then,

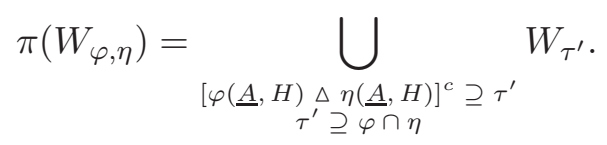

Furthermore, each fibre of $\pi: W_{\varphi, \eta} \rightarrow \bar{X}$ is affine and irreducible of dimension $g-\sharp(\varphi \cup \eta)$.

Proof. The proof is similar to the second proof appearing in Theorem 2.6.4. We know by Corollary 2.3.4 that $\pi\left(W_{\varphi, \eta}\right) \subseteq \bigcup_{\tau^{\prime}} W_{\tau^{\prime}}$, where $\tau^{\prime}$ is as above. Given a point $\bar{P}$ in $W_{\tau^{\prime}}$, where 
$[\varphi(\underline{A}, H) \Delta \eta(\underline{A}, H)]^{c} \supseteq \tau^{\prime} \supseteq \varphi \cap \eta$, consider the reduced fibre $\pi^{-1}(\bar{P})_{\text {red }}$. We want to construct a point $\mathbb{H}$ of the Grassmann variety $\mathscr{G}(\bar{P})$, such that $\mathbb{H}_{\beta}$ is a one dimensional subspace of $\mathbb{D}_{\beta}$, and

$$
\mathbb{H}_{\beta}= \begin{cases}\operatorname{Ker}(\operatorname{Ver})_{\beta} & \beta \in \varphi, \\ \operatorname{Ker}(\operatorname{Fr})_{\beta} & \beta \in \eta, \\ \notin\left\{\operatorname{Ker}(\operatorname{Fr})_{\beta}, \operatorname{Ker}(\operatorname{Ver})_{\beta}\right\} & \beta \notin \varphi \cup \eta .\end{cases}
$$

We need to check that $\mathbb{H}$ thus defined is indeed a point of $\mathscr{G}(\bar{P})$, which amounts to being stable under the maps Fr and Ver. This is a straightforward calculation:

- $\operatorname{Fr} \mathbb{H}_{\beta} \subseteq \mathbb{H}_{\sigma \circ \beta}$.

We distinguish cases:

(1) $\beta \in \eta$. Then this is clear as $\operatorname{Fr} \mathbb{H}_{\beta}=\{0\}$.

(2) $\beta \notin \eta$. In this case, since $\beta \in \ell(\varphi) \cup \eta$, it follows that $\beta \in \ell(\varphi)$, that is, $\sigma \circ \beta \in \varphi$. Then, Fr $\mathbb{H}_{\beta} \subseteq \operatorname{Ker}(\text { Ver })_{\sigma \circ \beta}=\mathbb{H}_{\sigma \circ \beta}$.

- $V \mathbb{H}_{\beta} \subseteq \mathbb{H}_{\sigma^{-1} \circ \beta}$.

The argument is entirely similar, where one distinguishes the cases: (i) $\beta \in \varphi$; (ii) $\beta \notin \varphi$, which implies $\sigma^{-1} \circ \beta \in \eta$.

We now show that

$$
\varphi(\mathbb{H})=\varphi, \quad \eta(\mathbb{H})=\eta .
$$

Clearly, $\varphi(\mathbb{H}) \supseteq \varphi$ and $\eta(\mathbb{H}) \supseteq \eta$. Note the following: (i) If $\beta \in(\varphi \cup \eta)^{c}$, then by definition $\mathbb{H}_{\beta} \neq \operatorname{Ker}(\operatorname{Ver})_{\beta}$, and so $\beta \notin \varphi(\mathbb{H})$; (ii) If $\beta \in \eta-\varphi$, then $\mathbb{H}_{\beta}=\operatorname{Ker}(\operatorname{Fr})_{\beta}$. On the other hand, by assumption, $\tau^{\prime} \cap(\eta-\varphi)=\emptyset$ and so $\operatorname{Ker}(\operatorname{Fr})_{\beta} \neq \operatorname{Ker}(\operatorname{Ver})_{\beta}$ and so $\mathbb{H}_{\beta} \neq \operatorname{Ker}(\operatorname{Ver})_{\beta}$. That is, $\beta \notin \varphi(\mathbb{H})$. Put together, these facts show that $\varphi(\mathbb{H})=\varphi$. A similar argument gives $\eta(\mathbb{H})=\eta$.

It follows from these considerations that $\pi^{-1}(\bar{P}) \cap W_{\varphi, \eta} \neq 0$. The above calculations show that if $\bar{P}$ is $k$-rational, then $\pi^{-1}(\bar{P}) \cap W_{\varphi, \eta}$ maps under $g$ to a closed subscheme of $\mathscr{G}$ isomorphic to $\mathbb{A}_{k}^{g-\sharp(\varphi \cup \eta)}$. This proves the second claim of the proposition (a finite morphism is affine).

2.7. The Atkin-Lehner automorphism. The Atkin-Lehner automorphism on $Y$ is the morphism

$$
w: Y \rightarrow Y
$$

characterized by its action on points:

$$
w(\underline{A}, H)=(\underline{A} / H, A[p] / H),
$$

equivalently,

$$
w\left(\left(\underline{A}, \alpha_{A}\right) \stackrel{f}{\longrightarrow}\left(\underline{B}, \alpha_{B}\right)\right)=\left(\left(\underline{B}, \alpha_{B}\right) \stackrel{f^{t}}{\longrightarrow}\left(\underline{A}, p \circ \alpha_{A}\right)\right) .
$$

Thus,

$$
w^{2}=\langle p\rangle,
$$

where $\langle p\rangle$ is the diamond operator whose effect on points is

$$
\left(\underline{A}, \alpha_{A}, H\right) \mapsto\left(\underline{A}, p \circ \alpha_{A}, H\right) .
$$


It follows that a power of $w^{2}$ is the identity and so $w$ is an automorphism of $Y$.

Proposition 2.7.1. The Atkin-Lehner automorphism acts on the stratification of $\bar{Y}$ by

$$
w\left(W_{\varphi, \eta}\right)=W_{r(\eta), \ell(\varphi)}, \quad w\left(Z_{\varphi, \eta}\right)=Z_{r(\eta), \ell(\varphi)} \cdot
$$

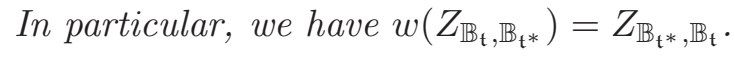

Proof. Suppose that $(f: \underline{A} \rightarrow \underline{B})$ is parameterized by a closed point $\bar{Q}$ in $W_{\varphi, \eta}$. Then

$$
\begin{aligned}
& \varphi(\bar{Q})=\left\{\beta \in B: \operatorname{Lie}(f)_{\sigma^{-1} \circ \beta}=0\right\}, \\
& \eta(\bar{Q})=\left\{\beta \in B: \operatorname{Lie}\left(f^{t}\right)_{\beta}=0\right\} .
\end{aligned}
$$

As $w\left(f:\left(\underline{A}, \alpha_{A}\right) \rightarrow\left(\underline{B}, \alpha_{B}\right)\right)=\left(f^{t}:\left(\underline{B}, \alpha_{B}\right) \rightarrow\left(\underline{A}, p \circ \alpha_{A}\right)\right)$,

$$
\begin{gathered}
\varphi(w(\bar{Q}))=\left\{\beta \in B: \operatorname{Lie}\left(f^{t}\right)_{\sigma^{-1} \circ \beta}=0\right\}=r(\eta(\bar{Q})), \\
\eta(\bar{Q})=\left\{\beta \in B: \operatorname{Lie}(f)_{\beta}=0\right\}=\ell(\varphi(\bar{Q})) .
\end{gathered}
$$

Lemma 2.7.2. For the choice of parameters in the uniformization 2.4.2) at $\bar{Q}$ and $w(\bar{Q})$, the homomorphism

$$
w^{*}: \widehat{\mathcal{O}}_{\bar{Y}, w(\bar{Q})} \longrightarrow \widehat{\mathcal{O}}_{\bar{Y}, \bar{Q}}
$$

is given by

$$
w^{*}\left(x_{\beta, w(\bar{Q})}\right)=y_{\beta, \bar{Q}}, \quad w^{*}\left(y_{\beta, w(\bar{Q})}\right)=x_{\beta, \bar{Q}}, \quad w^{*}\left(z_{\gamma, w(\bar{Q})}\right)=z_{\gamma, \bar{Q}},
$$

for $\beta \in I(\bar{Q})$, and $\gamma \notin I(\bar{Q})$.

Proof. We refer to the discussion before Theorem 2.4.1 in the following. In terms of the deformation theory discussed there, the morphism $w$ switches the role of $W_{A}^{R}$ and $W_{B}^{R}$ in a manner compatible with the $\mathcal{O}_{L}$-action. Therefore, it is clear that in the first formulation of Theorem 2.4 .1 we have $w^{*}\left(x_{\beta, w(\bar{Q})}\right)=y_{\beta, \bar{Q}}$ for $\beta \in \ell(\varphi(w(\bar{Q})))=\eta(\bar{Q})$, and $w^{*}\left(y_{\beta, w(\bar{Q})}\right)=x_{\beta, \bar{Q}}$ for $\beta \in \eta(w(\bar{Q}))=\ell(\varphi(\bar{Q}))$. The result now follows, since in the uniformization (2.4.2) the parameters $x_{\gamma, \bar{Q}}$ for $\gamma \in \ell(\varphi(\bar{Q}))-I(\bar{Q})$, and $y_{\gamma, \bar{Q}}$ for $\gamma \in \eta(\bar{Q})-I(\bar{Q})$ have been replaced with $z_{\gamma, \bar{Q}}$, and a similar reassignment has taken place at $w(\bar{Q})$.

Caveat. It is difficult for a point to $\bar{Q}$ to equal $w(\bar{Q})$; for that to happen, one must have, among other things, that $\bar{Q} \in \bar{Y}_{F} \cap \bar{Y}_{V}$ and $p \equiv 1(\bmod N)$. Nonetheless, if $\bar{Q}=w(\bar{Q})$, then it must be understood that in Lemma 2.7.2, we consider two possibly different sets of parameters at $\bar{Q}$ and $w(\bar{Q})$ despite the fact that our notation does not reflect that.

We will use the following lemma in the sequel. Let $\bar{Q} \in \bar{Y}_{F}$, and $\bar{P}=\pi(\bar{Q})$. Then $s(\bar{P})=\bar{Q}$.

Lemma 2.7.3. For the choice of parameters in the uniformization 2.4.2) at $\bar{Q}$, there is a choice of parameters as in (2.5.2) at $\bar{P}$ such that the homomorphism

$$
s^{*}: \widehat{\mathcal{O}}_{\bar{Y}, \bar{Q}} \longrightarrow \widehat{\mathcal{O}}_{\bar{X}, \bar{P}},
$$

is given by

$$
s^{*}\left(x_{\beta}\right)=t_{\beta}, \quad s^{*}\left(y_{\beta}\right)=0, \quad s^{*}\left(z_{\gamma}\right)=t_{\gamma},
$$

for $\beta \in I(\bar{Q})$, and $\gamma \notin I(\bar{Q})$. 
Proof. Let $\bar{P}$ correspond to $\underline{A}$; then $\bar{Q}$ corresponds to $\left(\mathrm{Fr}: \underline{A} \rightarrow \underline{A}^{(p)}\right)$, and we have $\varphi(\bar{Q})=\mathbb{B}$ and $I(\bar{Q})=\eta(\bar{Q})$. Fix an index $\beta \in \mathbb{B}$, and let $W_{1}, W_{2}, M, R$ be the data corresponding to $\bar{Q}$ "at the $\beta$-component," as in 2.4 . Applying deformation theory in a similar way, one can provide an isomorphism as in (2.5.2) at $\bar{P}$ which has the desired properties: the relevant Grassmann problem is to provide $W_{1}^{R}$ inside $R^{2}$ lifting $W_{1}$, and the parameter $t_{\beta}$ describes the deformation of $W_{1}$. Since, in the notation of the first formulation of Theorem 2.4.1, the parameter $x_{\beta}$ describes the deformation of $W_{1}$, we can choose $t_{\beta}$ such that $s^{*}\left(x_{\beta}\right)=t_{\beta}$. Note that in the isomorphism (2.4.2) $x_{\beta}$ is renamed to $z_{\beta}$ if $\beta \in I(\bar{Q})^{c}$, and that is how we have recorded this in the statement of the lemma. Finally, since $\operatorname{Fr}^{*}\left(\omega_{A^{(p)}}\right)=0$, any deformation of $W_{2}$ is constant, equal to $\operatorname{Ker}(M)$, and hence $s^{*}\left(y_{\beta}\right)=0$ whenever $y_{\beta}$ is defined, that is, for $\beta \in \eta(\bar{Q})=I(\bar{Q})$.

2.8. The infinitesimal nature of $\pi: \bar{Y} \rightarrow \bar{X}$. Let $k$ be a finite field containing $\kappa$, and $\bar{Q}$ a closed point of $\bar{Y}$ with residue field $k$. Let $\bar{P}=\pi(\bar{Q})$; let $\varphi=\varphi(\bar{Q}), \eta=\eta(\bar{Q}), I=I(\bar{Q})$, and $\tau=\tau(\bar{P})$. Choose the following isomorphisms

$$
\begin{aligned}
& \widehat{\mathcal{O}}_{\bar{Y}, \bar{Q}} \cong k \llbracket\left\{x_{\beta}, y_{\beta}: \beta \in I\right\},\left\{z_{\beta}: \beta \in I^{c}\right\} \rrbracket /\left(\left\{x_{\beta} y_{\beta}: \beta \in I\right\}\right), \\
& \widehat{\mathcal{O}}_{\bar{X}, \bar{P}} \cong k \llbracket t_{\beta}: \beta \in \mathbb{B} \rrbracket,
\end{aligned}
$$

as explained in $\S 22.42 .5$ The irreducible components of $\bar{Y}$ through $\bar{Q}$ are in bijection with subsets $J \subseteq I$. To such $J$, we have associated the ideal

$$
\mathcal{I}_{J}=\left\langle\left\{x_{\beta}: \beta \notin J\right\},\left\{y_{\beta} ; \beta \in J\right\}\right\rangle
$$

in $\widehat{\mathcal{O}}_{\bar{Y}}, \bar{Q}$. By Theorem 2.5.2, the closed set $V\left(\mathcal{I}_{J}\right)$ in Spf $\widehat{\mathcal{O}}_{\bar{Y}, \bar{Q}}$ corresponding to it is $Z_{r(\eta-J)^{c}, \eta-J}^{\wedge \bar{Q}}$, the formal completion of $Z_{r(\eta-J)^{c}, \eta-J}$ at $\bar{Q}$.

The following lemma, despite appearances, plays a key role in obtaining the results concerning the canonical subgroup. We shall refer to it in the sequel as "Key Lemma".

Lemma 2.8.1 (Key Lemma). Let $\beta \in \varphi \cap \eta$ and $\pi^{*}: \widehat{\mathcal{O}}_{\bar{X}, \bar{P}} \rightarrow \widehat{\mathcal{O}}_{\bar{Y}, \bar{Q}}$ the induced ring homomorphism.

(1) $\sigma \circ \beta \in \varphi, \sigma^{-1} \circ \beta \in \eta \quad$ In this case,

$$
\pi^{*}\left(t_{\beta}\right)=u x_{\beta}+v y_{\sigma^{-1} \circ \beta}^{p},
$$

for some units $u, v \in \widehat{\mathcal{O}}_{\bar{Y}}, \bar{Q}$.

(2) $\sigma \circ \beta \in \varphi, \sigma^{-1} \circ \beta \notin \eta \quad$ In this case,

$$
\pi^{*}\left(t_{\beta}\right)=u x_{\beta},
$$

for some unit $u \in \widehat{\mathcal{O}}_{\bar{Y}}, \bar{Q}$.

(3)

$\sigma \circ \beta \notin \varphi, \sigma^{-1} \circ \beta \in \eta$ In this case,

$$
\pi^{*}\left(t_{\beta}\right)=v y_{\sigma^{-1} \circ \beta}^{p},
$$

for some unit $v \in \widehat{\mathcal{O}}_{\bar{Y}, \bar{Q}}$. 
(4)

$\sigma \circ \beta \notin \varphi, \sigma^{-1} \circ \beta \notin \eta$
In this case,

$$
\pi^{*}\left(t_{\beta}\right)=0 .
$$

Proof. We first prove assertion (1). Fix $\beta$. We have

$$
\beta \in \varphi \cap \eta, \quad \sigma \circ \beta \in \varphi, \quad \sigma^{-1} \circ \beta \in \eta .
$$

Note that $\sigma \circ \beta \in \varphi \Rightarrow \beta \in \ell(\varphi) \Rightarrow \beta \in \ell(\varphi) \cap \eta=I$. Similarly, $\beta \in \varphi \Rightarrow \sigma^{-1} \circ \beta \in \ell(\varphi) \Rightarrow$ $\sigma^{-1} \circ \beta \in \ell(\varphi) \cap \eta=I$. That is to say, both $\beta$ and $\sigma^{-1} \circ \beta$ are critical indices.

Let $J \subseteq I, \varphi_{0}=r(\eta-J)^{c}, \eta_{0}=\eta-J$. Then $V\left(\mathcal{I}_{J}\right)=Z_{\varphi_{0}, \eta_{0}}^{\wedge \bar{Q}}$. In the following analysis, divided into three cases, we obtain information about $\pi^{*}\left(t_{\beta}\right)$ modulo various ideals $\mathcal{I}_{J}$, which is then assembled to produce the final result $\pi^{*}\left(t_{\beta}\right)=u x_{\beta}+v y_{\sigma^{-1} \circ \beta}^{p}$. The three cases do not cover all possibilities, but they suffice for the following.

CASE A: $\left\{\sigma^{-1} \circ \beta, \beta\right\} \subseteq J$.

Since $\sigma^{-1} \circ \beta \in J$ we have $\beta \in r(J)$ and, since $\varphi_{0}=r\left(\eta^{c}\right) \cup r(J)$, we have $\beta \in \varphi_{0}$. Therefore, $\beta \in \varphi\left(\bar{Q}^{\prime}\right)$ for any point $\bar{Q}^{\prime} \in Z_{\varphi_{0}, \eta_{0}}$ and so, by Corollary 2.3.4, $\beta \in \tau\left(\bar{Q}^{\prime}\right)$ if and only if $\beta \in \eta\left(\bar{Q}^{\prime}\right)$. This can be rephrased as saying that the vanishing locus of $\pi^{*}\left(t_{\beta}\right)$ on $Z_{\varphi_{0}, \eta_{0}}^{\wedge \bar{Q}}$ lies inside the closed formal subscheme $V_{\beta}^{+} \cap Z_{\varphi_{0}, \eta_{0}}^{\wedge \bar{Q}}\left(V_{\beta}^{+}\right.$was defined in Lemma 2.4.2), which in the completed local ring is defined by the vanishing of $x_{\beta}$. This implies that

$$
x_{\beta} \in \sqrt{\left\langle\pi^{*}\left(t_{\beta}\right)\right\rangle}
$$

in the ring

$$
\widehat{\mathcal{O}}_{\bar{Y}, \bar{Q}} / \mathcal{I}_{J} \cong k \llbracket\left\{x_{\gamma}: \gamma \in J\right\},\left\{y_{\gamma}: \gamma \in I-J\right\},\left\{z_{\gamma}: \gamma \in \mathbb{B}-I\right\} \rrbracket,
$$

which is a power-series ring. Therefore, there exists a positive integer $M(J)$ such that

$$
\pi^{*}\left(t_{\beta}\right)-u(J) x_{\beta}^{M(J)} \in \mathcal{I}_{J}
$$

for $u(J)$ a (lift of a unit $\bmod \mathcal{I}_{J}$ and hence a) unit in $\widehat{\mathcal{O}}_{\bar{Y}, \bar{Q}}$.

CASE B: $\left\{\sigma^{-1} \circ \beta, \beta\right\} \subseteq I-J$.

Since $\beta \in \eta$, we have $\beta \in \eta-J=\eta_{0}$ in this case. On the other hand, $\sigma^{-1} \circ \beta \notin J$ and so $\beta \notin r(J)$, and also $\sigma^{-1} \circ \beta \in \eta$ and so $\beta \notin r(\eta)^{c}$. Together these imply that $\beta \notin r(\eta)^{c} \cup r(J)=\varphi_{0}$. Arguing as in CASE A (where we had $\beta \in \varphi_{0}, \beta \notin \eta_{0}$ ), we deduce that there is a positive integer $N(J)$ such that

$$
\pi^{*}\left(t_{\beta}\right)-v(J) y_{\sigma^{-1} \circ \beta}^{N(J)} \in \mathcal{I}_{J},
$$

for some unit $v(J) \in \widehat{\mathcal{O}}_{\bar{Y}, \bar{Q}}$.

CASE C: $\sigma^{-1} \circ \beta \in J, \beta \notin J$.

The assumption implies that $\beta \in r(J)$ and so $\beta \in \varphi_{0}$. Also, $\beta \in I-J \subseteq \eta-J$ and so $\beta \in \eta_{0}$. This implies that $\beta \in \varphi\left(\bar{Q}^{\prime}\right) \cap \eta\left(\bar{Q}^{\prime}\right)$ for any closed point $\bar{Q}^{\prime} \in Z_{\varphi_{0}, \eta_{0}}$ and hence that $\pi\left(Z_{\varphi_{0}, \eta_{0}}\right) \subseteq Z_{\{\beta\}}$. Therefore, $\pi^{*}\left(t_{\beta}\right)$ vanishes identically on $Z_{\varphi_{0}, \eta_{0}}$, that is,

$$
\pi^{*}\left(t_{\beta}\right) \in \mathcal{I}_{J}
$$


As mentioned above the remaining case, $\sigma^{-1} \circ \beta \notin J, \beta \in J$, is not needed in the following. We now proceed with the proof of assertion (1).

Taking $J=I$ and $J=\emptyset$, we find $M=M(I), u$ and $N=N(\emptyset), v$, as in CASE A and CASE B, respectively. We can therefore write

$$
\pi^{*}\left(t_{\beta}\right)=u x_{\beta}^{M}+v y_{\sigma^{-1} \circ \beta}^{N}+E,
$$

where the "error term" $E \in \mathcal{I}_{I} \cap \mathcal{I}_{\emptyset} \subset \widehat{\mathcal{O}}_{\bar{Y}}, \bar{Q}$. Reducing this equation modulo $\mathcal{I}_{J}$ for every $J$ falling under CASE C, we find, using Lemma 2.8.2 below, that

$$
E \in \bigcap_{\substack{J \subseteq I, \beta \notin J, \sigma^{-1} \circ \beta \in J .}} \mathcal{I}_{J}=\left\langle x_{\beta}, y_{\sigma^{-1} \circ \beta}\right\rangle .
$$

Therefore, we may write $E=A x_{\beta}+B y_{\sigma^{-1} \circ \beta}$ and, by reducing modulo $\mathcal{I}_{I}$ and $\mathcal{I}_{\emptyset}$, we find that

$$
\pi^{*}\left(t_{\beta}\right)=u x_{\beta}^{M}+v y_{\sigma^{-1} \circ \beta}^{N}+A x_{\beta}+B y_{\sigma^{-1} \circ \beta}, \quad A \in\left\langle y_{\gamma}: \gamma \in I\right\rangle, B \in\left\langle x_{\gamma}: \gamma \in I\right\rangle .
$$

Choosing $J$ as in CASE B, we can find $N(J), v(J)$, such that $\pi^{*}\left(t_{\beta}\right)-v(J) \cdot y_{\sigma^{-1} \circ \beta}^{N(J)} \in \mathcal{I}_{J}$. Hence, we find that $v y_{\sigma^{-1} \circ \beta}^{N}+B y_{\sigma^{-1} \circ \beta}=v(J) y_{\sigma^{-1} \circ \beta}^{N(J)}$ in the local ring

$$
R_{J}=\widehat{\mathcal{O}}_{\bar{Y}, \bar{Q}} / \mathcal{I}_{J}=k \llbracket\left\{x_{\gamma}: \gamma \in J\right\},\left\{y_{\gamma}: \gamma \in I-J\right\},\left\{z_{\gamma}: \gamma \in \mathbb{B}-I\right\} \rrbracket .
$$

Let $v_{0}$ and $v_{0}(J)$ denote, respectively, the constant term in $v$ and $v(J)$ in the power-series ring $R_{J}$. Because $B \in\left\langle x_{\gamma}: \gamma \in I\right\rangle$, it follows that $v_{0}=v_{0}(J)$ and $N=N(J)$. That implies that $B \in\left\langle y_{\sigma^{-1} \circ \beta}^{N-1}\right\rangle+\mathcal{I}_{J}$ for all $J$ as in CASE B. Therefore,

$$
\left.B \in \bigcap_{J \subseteq I}\left\langle y_{\sigma^{-1} \circ \beta}^{N-1}\right\rangle+\mathcal{I}_{J}=\left\langle y_{\sigma^{-1} \circ \beta}^{N-1}, x_{\sigma^{-1} \circ \beta}, x_{\beta}\right\rangle, \quad \text { (By Lemma 2.8.2. }\right)
$$

Write $B=a y_{\sigma^{-1} \circ \beta}^{N-1}+b x_{\sigma^{-1} \circ \beta}+c x_{\beta}$, where $a \in\left\langle x_{\beta}: \beta \in I\right\rangle$. Hence:

$$
\pi^{*}\left(t_{\beta}\right)=u x_{\beta}^{M}+v y_{\sigma^{-1} \circ \beta}^{N}+A x_{\beta}+a y_{\sigma^{-1} \circ \beta}^{N}+c x_{\beta} y_{\sigma^{-1} \circ \beta} .
$$

To proceed, we need a sub-lemma.

Sub-lemma. $M=1, N=p$.

Proof. (Sub-lemma) First we prove the statement for $\bar{Q} \in \bar{Y}_{F} \cap \bar{Y}_{V}$. At such a point $\bar{Q}, \varphi(\bar{Q})=$ $\eta(\bar{Q})=\mathbb{B}=I$. Since $\bar{Y}_{F}=Z_{\mathbb{B}, \emptyset}$, it follows that $V\left(\mathcal{I}_{I}\right)$ is the image of $\bar{Y}_{F}$ in $\operatorname{Spf}\left(\widehat{\mathcal{O}}_{\bar{Y}}, \bar{Q}\right)$. Since there is a section to $\left.\pi\right|_{\bar{Y}_{F}}$, we find that $\pi^{*}\left(Z_{\{\beta\}}\right)$ is a reduced Weil divisor on $\bar{Y}_{F}$, and hence $\pi^{*}\left(t_{\beta}\right)=u x_{\beta}^{M}$ in $\widehat{\mathcal{O}}_{\bar{Y}, \bar{Q}} / \mathcal{I}_{I}$ implies that $M=1$. A similar argument using $\bar{Y}_{V}$ and applying Proposition 2.8.3 gives that $N=p$.

Now for the general case: the point $\bar{Q}$ belongs to $Z_{\varphi_{0}, \eta_{0}}=Z_{r(\eta)^{c} \cup r(J), \eta-J}$ for $J=I$, and so it belongs to an irreducible component $C$ of $Z_{\varphi_{0}, \eta_{0}}$. Let $D$ be the pull-back of the Cartier divisor $Z_{\{\beta\}}$, under $\pi: C \rightarrow \bar{X}$. Since $\beta \in \varphi(\bar{Q}) \cap \eta(\bar{Q})=\varphi \cap \eta$, but $\beta \in \varphi_{0}-\eta_{0}$, we see that $D$ is 
a non-zero Cartier divisor on $C$ containing $\bar{Q}$, and since $C$ is non-singular, in fact a non-zero effective Weil divisor on $C$. Let us write then

$$
D=n_{1} D_{1}+n_{2} D_{2}+\cdots+n_{r} D_{r},
$$

a sum of irreducible Weil divisors $D_{i}$, and say $\bar{Q} \in D_{1}$. Since $Z_{\{\beta\}}$ is given formally locally by the vanishing of $t_{\beta}$, we see that $D$ at $\bar{Q}$ is given by the vanishing of $\pi^{*}\left(t_{\beta}\right)$ modulo $\mathcal{I}_{I}$, which is equal to $u x_{\beta}^{M}$. This, in turn, implies that $D$ is locally irreducible and hence $\bar{Q}$ belongs only to $D_{1}$ and $M=n_{1}$.

Sub-sub-lemma. $D_{1} \cap \bar{Y}_{F} \cap \bar{Y}_{V} \neq \emptyset$.

Proof. (Sub-sub-lemma) We have $\beta \in \varphi_{0}$ and so $\beta \in \varphi(\bar{R})$ for any closed point $\bar{R} \in Z_{\varphi_{0}, \eta_{0}}$. Therefore, $\pi(\bar{R}) \in Z_{\{\beta\}}$ if and only if $\beta \in \eta(\bar{R})$ (Corollary 2.3.4). Therefore, at least set theoretically,

$$
Z_{\varphi_{0}, \eta_{0}} \cap \pi^{-1}\left(Z_{\{\beta\}}\right)=Z_{\varphi_{0}, \eta_{0} \cup\{\beta\}} .
$$

Since $Z_{\varphi_{0}, \eta_{0}}$ is a disjoint union of its components, every irreducible component of $C \cap \pi^{-1}\left(Z_{\{\beta\}}\right)$ (i.e., $\left.D_{1}, \ldots, D_{r}\right)$ is an irreducible component of $Z_{\varphi_{0}, \eta_{0} \cup\{\beta\}}$. By Theorem 2.6.13, every such irreducible component contains a point $\bar{R}$ that also belongs to $\bar{Y}_{F} \cap \bar{Y}_{V}$. That ends the proof of the sub-sub-lemma.

Back to the proof of the sub-lemma. Let $\bar{R} \in D_{1} \cap \bar{Y}_{F} \cap \bar{Y}_{V}$ be a closed point. Repeating the argument above now for the point $\bar{R}$, we find that $M=n_{1}=M(\bar{R})$, where $M(\bar{R})$ is the exponent $M$ occurring in Equation (2.8.3) at the point $\bar{R}$. Using the argument already done for the special case of points on $\bar{Y}_{F} \cap \bar{Y}_{V}$, we conclude that $M(\bar{R})=1$ and hence $M=1$. A similar argument gives that $N=p$ and this concludes the proof of the Sub-lemma.

We can now refine Equation (2.8.3) and write

$$
\pi^{*}\left(t_{\beta}\right)=\left(u+A+c y_{\sigma^{-1} \circ \beta}\right) x_{\beta}+(v+a) y_{\sigma^{-1} \circ \beta}^{p} .
$$

Note that both $u^{\prime}:=u+A+c y_{\sigma^{-1} \circ \beta}$ and $v^{\prime}:=v+a$ are units in $\widehat{\mathcal{O}}_{\bar{Y}, \bar{Q}}$ as $A+c y_{\sigma^{-1} \circ \beta}$ and $a$ are in the maximal ideal and $u, v$ are units. Thus,

$$
\pi^{*}\left(t_{\beta}\right)=u^{\prime} x_{\beta}+v^{\prime} y_{\sigma^{-1} \circ \beta}^{p},
$$

concluding the proof of assertion (1) of the Lemma.

Assertions (2) and (3) are proved in a similar fashion and the argument, if anything, is easier. We discuss now assertion (4). Recall that in this case,

$$
\beta \in \varphi \cap \eta, \quad \sigma \circ \beta \notin \varphi, \quad \sigma^{-1} \circ \beta \notin \eta .
$$

This implies that

$$
\left\{\sigma^{-1} \circ \beta, \beta\right\} \subseteq I^{c}
$$

because $\beta \notin \ell(\varphi) \Rightarrow \beta \notin I$ and $\sigma^{-1} \circ \beta \notin \eta \Rightarrow \sigma^{-1} \circ \beta \notin I$. Consequently, for every $J \subseteq I$, $\beta \in \eta-J=\eta_{0}$ and also $\beta \in r(\eta)^{c} \subseteq r(\eta)^{c} \cup r(J)=\varphi_{0}$. Thus, $\beta \in \varphi_{0} \cap \eta_{0}$ on any such $Z_{\varphi_{0}, \eta_{0}}$, which implies that $\pi^{*}\left(t_{\beta}\right)=0 \bmod \mathcal{I}_{J}$ for all $J \subseteq I$. Since these correspond to all the irreducible components through $\bar{Q}, \pi^{*}\left(t_{\beta}\right)=0$. 
During the proof of the Key-Lemma we have used the following result.

Lemma 2.8.2. Let $I$ be a subset of $\mathbb{B}$ and consider the ring $k \llbracket\left\{x_{\beta}: \beta \in I\right\},\left\{y_{\beta}: \beta \in I\right\},\left\{z_{\beta}\right.$ : $\beta \in \mathbb{B}-I\} \rrbracket /\left\langle\left\{x_{\beta} y_{\beta}: \beta \in I\right\}\right\rangle$ and for $J \subseteq I$ its ideal $\mathcal{I}_{J}=\left\langle\left\{x_{\beta}: \beta \notin J\right\},\left\{y_{\beta} ; \beta \in J\right\}\right\rangle$. Then:

$$
\begin{aligned}
& \bigcap_{\substack{J \subseteq I, \beta \notin J, \sigma^{-1} \circ \beta \in J .}} \mathcal{I}_{J}=\left\langle x_{\beta}, y_{\sigma^{-1} \circ \beta}\right\rangle . \\
& \bigcap_{\substack{J \subseteq I \\
\left\{\sigma^{-1} \circ \beta, \beta\right\} \subseteq I-J}}\left\langle y_{\sigma^{-1} \circ \beta}^{N-1}\right\rangle+\mathcal{I}_{J}=\left\langle y_{\sigma^{-1} \circ \beta}^{N-1}, x_{\sigma^{-1} \circ \beta}, x_{\beta}\right\rangle .
\end{aligned}
$$

Proof. Consider the power series ring

$$
R=k \llbracket x_{\beta}, y_{\beta}, z_{\gamma} \rrbracket_{\beta \in I, \gamma \in I^{c}}
$$

and the preimage of the above ideals, under the natural projection,

$$
\mathcal{I}_{J}^{0}=\left\langle\left\{x_{\beta}: \beta \notin J\right\},\left\{y_{\beta} ; \beta \in J\right\}\right\rangle, \quad \mathcal{I}_{J}^{1}=\left\langle y_{\sigma^{-1} \circ \beta}^{N-1}\right\rangle+\mathcal{I}_{J}^{0} .
$$

It is enough to show that in $R$ we have

$$
\begin{aligned}
& \bigcap_{\substack{J \subseteq I, \beta \notin J, \sigma^{-1} \circ \beta \in J .}} \mathcal{I}_{J}^{0}=\left\langle x_{\beta}, y_{\sigma^{-1} \circ \beta},\left\{x_{\gamma} y_{\gamma}: \gamma \in I\right\}\right\rangle . \\
& \bigcap_{\substack{J \subseteq I \\
\left\{\sigma^{-1} \circ \beta, \beta\right\} \subseteq I-J}} \mathcal{I}_{J}^{1}=\left\langle y_{\sigma^{-1} \circ \beta}^{N-1}, x_{\sigma^{-1} \circ \beta}, x_{\beta},\left\{x_{\gamma} y_{\gamma}: \gamma \in I\right\}\right\rangle .
\end{aligned}
$$

Recall that a monomial ideal of $R$ is an ideal generated by monomials. The ideals $\mathcal{I}_{J}^{0}, \mathcal{I}_{J}^{1}$ are monomial ideals. The statements follow easily from the following result. Let $\mathfrak{a}=\left\langle f_{1}, \ldots, f_{a}\right\rangle, \mathfrak{b}=$ $\left\langle g_{1}, \ldots, g_{b}\right\rangle$, be two monomial ideals of $R$ then $\mathfrak{a} \cap \mathfrak{b}$ is a monomial ideal of $R$ and

$$
\mathfrak{a} \cap \mathfrak{b}=\left\langle\left\{\operatorname{lcm}\left(f_{i}, g_{j}\right): 1 \leq i \leq a, 1 \leq j \leq b\right\}\right\rangle
$$

(cf. [15, §15, Exercise 15.7]).

At a point $\bar{Q}$, the Key Lemma gives information only about $\pi^{*}\left(t_{\beta}\right)$ with $\beta \in \varphi(\bar{Q}) \cap \eta(\bar{Q})$. If no such $\beta$ exists, that is, if $\varphi(\bar{Q}) \cap \eta(\bar{Q})=\emptyset$, then the admissibility condition implies that

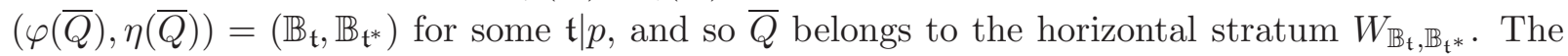
following lemma studies this situation; it was in fact used in the proof of the Key Lemma.

In the case at hand, we have $I(\bar{Q})=\emptyset$, and hence the isomorphism (2.8.1) becomes

$$
\widehat{\mathcal{O}}_{\bar{Y}, \bar{Q}} \cong k \llbracket z_{\beta}: \beta \in \mathbb{B} \rrbracket .
$$

Proposition 2.8.3. Let $\bar{Q} \in W_{\mathbb{B}_{\mathfrak{t}}, \mathbb{B}_{\mathfrak{t}^{*}}}$, and $\bar{P}=\pi(\bar{Q})$. We can choose isomorphisms as in (2.8.2) at $\bar{P}$, and 2.8.4 at $\bar{Q}$, such that:

$$
\pi^{*}\left(t_{\beta}\right)= \begin{cases}z_{\beta} & \beta \in \mathbb{B}_{\mathfrak{t}} \\ z_{\beta}^{p} & \beta \in \mathbb{B}_{\mathfrak{t}^{*}} .\end{cases}
$$

Proof. See Appendix, Lemma 7.1.2.

Remark 2.8.4. To prove the sub-lemma that appeared in the proof of the Key Lemma, we appealed to Theorem 2.6.13. We remark that the sublemma can also be proven directly from Proposition 2.8.3, by considering the horizontal strata that pass through the point $\bar{Q}$. There, we only used 
information concerning the infinitesimal description of $\pi$ on two horizontal strata, that is $\bar{Y}_{F}$ and $\bar{Y}_{V} ;$ this is a special case of Proposition 2.8.3.

\section{Extension to the Cusps}

3.1. Notation. We will let $\mathbb{X}$ denote the minimal compactification of $X$ defined over $W(\kappa)$, and $\overline{\mathbb{X}}$ its special fibre over $\kappa$ (see [8] and the references therein). Define $\mathbb{Y}, \overline{\mathbb{Y}}$ similarly. The morphisms $\pi, w$, and $s$ extend to morphisms $\pi: \mathbb{Y} \rightarrow \mathbb{X}, w: \mathbb{Y} \rightarrow \mathbb{Y}$, and $s: \overline{\mathbb{X}} \rightarrow \overline{\mathbb{Y}}$; Reducing modulo $p$, we obtain $\pi: \overline{\mathbb{Y}} \rightarrow \overline{\mathbb{X}}$.

3.2. Extension of the stratification. Let $\tau \subseteq \mathbb{B}$. We define $\mathbb{W}_{\tau}=W_{\tau}$ and $\mathbb{Z}_{\tau}=Z_{\tau}$, unless $\tau=\emptyset$, in which case we set $\mathbb{W}_{\emptyset}=W_{\emptyset} \cup(\overline{\mathbb{X}}-\bar{X})$ and $\mathbb{Z}_{\emptyset}=\overline{\mathbb{X}}$. The collection $\left\{\mathbb{W}_{\tau}\right\}_{\tau \subseteq \mathbb{B}}$ is a stratification of $\overline{\mathbb{X}}$. In fact, we find that $\mathbb{Z}_{\tau}$ is the Zariski closure of $Z_{\tau}$ in $\overline{\mathbb{X}}$. This follows from the fact that $\cup_{\tau \neq \emptyset} Z_{\tau}$ is Zariski closed in $\overline{\mathbb{X}}$ as it is the union of the vanishing loci of the partial Hasse invariants which have constant $q$-expansion at infinity $[18$. For a point $\bar{P} \in \overline{\mathbb{X}}-\bar{X}$, we define $\tau_{\beta}(\bar{P})=0$ for all $\beta \in \mathbb{B}$.

For an admissible pair $(\varphi, \eta)$, define $\mathbb{Z}_{\varphi, \eta}$ to be the Zariski closures of $Z_{\varphi, \eta}$ in $\bar{Y}$. We define $\mathbb{W}_{\varphi, \eta}=\mathbb{Z}_{\varphi, \eta}-\cup_{\left(\varphi^{\prime}, \eta^{\prime}\right)>(\varphi, \eta)} \mathbb{Z}_{\varphi^{\prime}, \eta^{\prime}}$.

Theorem 3.2.1. Let $(\varphi, \eta)$ be an admissible pair.

(1) $\mathbb{W}_{\varphi, \eta}=W_{\varphi, \eta}$ and $\mathbb{Z}_{\varphi, \eta}=Z_{\varphi, \eta}$, unless there is $\mathfrak{t} \mid p$ such that $(\varphi, \eta)=\left(\mathbb{B}_{\mathfrak{t}}, \mathbb{B}_{\mathfrak{t}^{*}}\right)$, in which case $\mathbb{W}_{\varphi, \eta}-W_{\varphi, \eta}=\mathbb{Z}_{\varphi, \eta}-Z_{\varphi, \eta}$ is non-empty, lies inside $\overline{\mathbb{Y}}-\bar{Y}$, and consists of finitely many cusps if $\overline{\mathbb{Y}}=\mathrm{m} \overline{\mathbb{Y}}$.

(2) $\operatorname{dim}\left(\mathbb{W}_{\varphi, \eta}\right)=\operatorname{dim}\left(W_{\varphi, \eta}\right)=2 g-(\sharp \varphi+\sharp \eta)$.

(3) The irreducible components of $\overline{\mathbb{Y}}$ are the irreducible components of the strata $\mathbb{Z}_{\varphi, \ell\left(\varphi^{c}\right)}$ for $\varphi \subseteq \mathbb{B}$.

(4) If $\left(\varphi^{\prime}, \eta^{\prime}\right)$ is another admissible pair, then $\mathbb{Z}_{\varphi, \eta} \cap \mathbb{Z}_{\varphi^{\prime}, \eta^{\prime}}=Z_{\varphi, \eta} \cap Z_{\varphi^{\prime}, \eta^{\prime}}$.

(5) The collection $\left\{\mathbb{W}_{\varphi, \eta}\right\}_{\varphi, \eta}$ is a stratification of $\overline{\mathbb{Y}}$.

Proof. For an admissible pair $(\varphi, \eta)$, we have $\varphi \cap \eta=\emptyset$ if and only if $(\varphi, \eta)=\left(\mathbb{B}_{\mathfrak{t}}, \mathbb{B}_{\mathfrak{t}^{*}}\right)$ for some $\mathfrak{t} \mid p$. This is because, by assumptions, $\ell\left(\varphi^{c}\right) \subseteq \eta \subseteq \varphi^{c}$, and hence $\ell(\varphi)=\varphi$ and $\eta=\varphi^{c}$, implying $\varphi=\mathbb{B}_{\mathfrak{t}}$ and $\eta=\mathbb{B}_{\mathfrak{t}^{*}}$ for some $\mathfrak{t} \mid p$. Let $Z=\cup_{\varphi \cap \eta \neq \emptyset} Z_{\varphi, \eta}$. We have

$$
\overline{\mathbb{Y}}-Z=\bigcup_{\mathfrak{t} \mid p} W_{\mathbb{B}_{\mathfrak{t}}, \mathbb{B}_{\mathfrak{t}^{*}}} \cup(\overline{\mathbb{Y}}-\bar{Y}) .
$$

We have $\pi(Z) \subseteq \cup_{\tau \neq \emptyset} Z_{\tau}$ by part (1) of Corollary 2.3.4. Also, since $\pi(\overline{\mathbb{Y}}-\bar{Y})=\overline{\mathbb{X}}-\bar{X}$, and by Corollary 2.3.4, we see that $\pi(\overline{\mathbb{Y}}-Z)$ lies in $\mathbb{W}_{\emptyset}$. Therefore, $Z=\pi^{-1}\left(\cup_{\tau \neq \emptyset} Z_{\tau}\right)=\pi^{-1}\left(\cup_{\tau \neq \emptyset} \mathbb{Z}_{\tau}\right)$, and in particular, $Z$ is Zariski closed in $\overline{\mathbb{Y}}$. This proves (1).

Statement (2) is clear from part (2) of Theorem 2.5.2. Statement (3) follows from part (3) of Theorem 2.5.2, since the irreducible components of $\bar{Y}$ are the Zariski closures of the irreducible components of $\bar{Y}$ in $\overline{\mathbb{Y}}$. Statement (5) is clear from the definition. 
We prove $(4)$ in the case where $(\varphi, \eta)=\left(\mathbb{B}_{\mathfrak{t}}, \mathbb{B}_{\mathfrak{t}^{*}}\right)$ and $\left(\varphi^{\prime}, \eta^{\prime}\right)=\left(\mathbb{B}_{\mathfrak{z}}, \mathbb{B}_{\mathfrak{z}^{*}}\right)$ for two distinct ideals

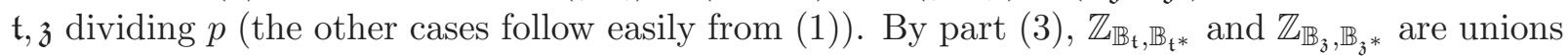
of irreducible components of $\overline{\mathbb{Y}}$. Since $\mathfrak{t} \neq \mathfrak{z}, \mathbb{Z}_{\mathbb{B}_{\mathfrak{t}}, \mathbb{B}_{\mathfrak{t}^{*}}}$ and $\mathbb{Z}_{\mathbb{B}_{\mathfrak{z}}, \mathbb{B}_{\mathfrak{z}^{*}}}$ have no irreducible components in common, as their intersection has dimension strictly less than each of them. Since $\overline{\mathbb{Y}}$ is integral

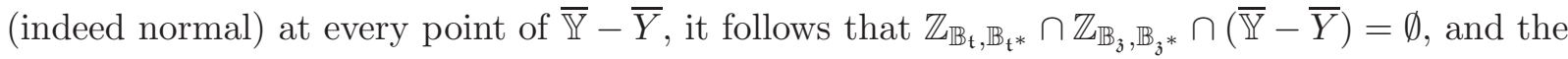
result follows.

Definition 3.2.2. By Theorem 3.2.1, every closed point $\bar{Q}$ in $\bar{Y}-\bar{Y}$ belongs to a unique horizontal

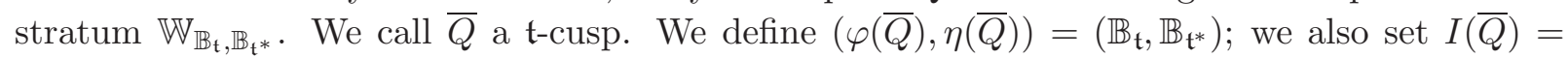
$\ell(\varphi(\bar{Q})) \cap \eta(\bar{Q})=\emptyset$. Note that in this case, $(\varphi(\bar{Q}), \eta(\bar{Q}))$ is an admissible pair.

Proposition 3.2.3. If $\bar{Q}$ is a $\mathfrak{t}$-cusp, then $w(\bar{Q})$ is a $\mathfrak{t}^{*}$-cusp.

Proof. This follows from Proposition 2.7.1

Given $\varphi \subseteq \mathbb{B}$, define $\mathbb{U}_{\varphi}^{+}$to be the Zariski closure of $U_{\varphi}^{+}$in $\overline{\mathbb{Y}}$. Similarly, for $\eta \subseteq \mathbb{B}$, define $\mathbb{V}_{\eta}^{+}$ to be the Zariski closure of $V_{\eta}^{+}$in $\overline{\mathbb{Y}}$.

Lemma 3.2.4. Let $\varphi$ and $\eta$ be subsets of $\mathbb{B}$.

(1) $\mathbb{U}_{\varphi}^{+}$is the closed subset of $\overline{\mathbb{Y}}$ consisting of points $\bar{Q}$ with $\varphi(\bar{Q}) \supseteq \varphi$. Similarly, $\mathbb{V}_{\eta}^{+}$is the closed subset of $\overline{\mathbb{Y}}$ consisting of points $\bar{Q}$ with $\eta(\bar{Q}) \supseteq \eta$

(2) Assume $\beta \in \mathbb{B}$. If $\bar{Q} \in \mathbb{U}_{\{\beta\}}^{+}$, then $\mathbb{U}_{\{\beta\}}^{+} \cap \operatorname{Spf}\left(\widehat{\mathcal{O}}_{\bar{Y}, \bar{Q}}\right)$ is equal to $\operatorname{Spf}\left(\widehat{\mathcal{O}}_{\bar{Y}, \bar{Q}}\right)$ if $\beta \notin r(I(\bar{Q}))$, and is otherwise given by the vanishing of $y_{\sigma^{-1} \circ \beta}$. If $\bar{Q} \in \mathbb{V}_{\{\beta\}}^{+}$, then $\mathbb{V}_{\{\beta\}}^{+} \cap \operatorname{Spf}\left(\widehat{\mathcal{O}}_{\bar{Y}, \bar{Q}}\right)$ is equal to $\operatorname{Spf}\left(\widehat{\mathcal{O}}_{\bar{Y}, \bar{Q}}\right)$ if $\beta \notin I$, and is otherwise given by the vanishing of $x_{\beta}$.

Proof. Statement (1) follows from part (1) of Theorem 3.2.1. To prove (2), it is enough to consider $\bar{Q} \in \overline{\mathbb{Y}}-\bar{Y}$; the rest is covered by Lemma 2.4.2. In that case, there is an ideal gert $\mid p$ such that $(\varphi(\bar{Q}), \eta(\bar{Q}))=\left(\mathbb{B}_{\mathfrak{t}}, \mathbb{B}_{\mathfrak{t}^{*}}\right), I(\bar{Q})=\emptyset$, and $\beta \in \mathbb{B}_{\mathfrak{t}}$. Then, $\mathbb{W}_{\mathbb{B}_{\mathfrak{t}}, \mathbb{B}_{\mathfrak{t}^{*}}}$ is a Zariski open subset of $\overline{\mathbb{Y}}$ (being the complement of $\left.\cup_{(\varphi, \eta) \neq\left(\mathbb{B}_{\mathfrak{t}}, \mathbb{B}_{\mathfrak{t}^{*}}\right)} \mathbb{Z}_{\varphi, \eta}\right)$ containing $\bar{Q}$ which lies entirely in $\mathbb{U}_{\{\beta\}}^{+}$. This implies that $\mathbb{U}_{\{\beta\}}^{+} \cap \operatorname{Spf}\left(\widehat{\mathcal{O}}_{\bar{Y}, \bar{Q}}\right)$ is equal to $\operatorname{Spf}\left(\widehat{\mathcal{O}}_{\bar{Y}, \bar{Q}}\right)$.

Definition 3.2.5. Let $\overline{\mathbb{X}}^{\text {ord }}=\mathbb{W}_{\emptyset}$ and $\overline{\mathbb{Y}}^{\text {ord }}=\cup_{\mathfrak{t} \mid p} \mathbb{W}_{\mathbb{B}_{\mathfrak{t}}, \mathbb{B}_{\mathfrak{t}^{*}}}$. We define $\overline{\mathbb{Y}}_{F}^{\text {ord }}$ to be $\mathbb{W}_{\mathbb{B}, \emptyset}$; it is the image of $\overline{\mathbb{X}}^{\text {ord }}$ under the section $s$. We define $\overline{\mathbb{Y}}_{V}^{\text {ord }}$ to be $\mathbb{W}_{\emptyset, \mathbb{B}}$; it is equal to $w\left(\overline{\mathbb{Y}}_{F}^{\text {ord }}\right)$. Compare with Proposition 2.5.5.

\section{Valuations And a dissection of $\mathfrak{Y}_{\text {rig }}$}

4.1. Notation. We denote the completions of $\mathbb{X}, \mathbb{Y}$ along their special fibres, respectively, by $\mathfrak{X}, \mathfrak{Y}$. These are quasi-compact quasi-separated topologically finitely generated (i.e., admissible) formal schemes over $W(\kappa)$. By Raynaud's work, one can associate to them their rigid analytic generic fibres, respectively, $\mathfrak{X}_{\text {rig }}, \mathfrak{Y}_{\text {rig }}$, which are quasi-compact, quasi-separated rigid analytic varieties over $\mathbb{Q}_{\kappa}$. Since $\mathbb{X}, \mathbb{Y}$ are proper over $W(\kappa)$, one sees that $\mathfrak{X}_{\text {rig }}, \mathfrak{Y}_{\text {rig }}$ are in fact, respectively, the analytifications of $\mathbb{X} \otimes_{W(\kappa)} \mathbb{Q}_{\kappa}, \mathbb{Y} \otimes_{W(\kappa)} \mathbb{Q}_{\kappa}$. Note that these spaces have natural models 
defined over $\mathbb{Q}_{p}$, which we denote, respectively, by $\mathfrak{X}_{\text {rig, } \mathbb{Q}_{p}}, \mathfrak{Y}_{\text {rig, } \mathbb{Q}_{p}}$. We say that a point $P \in \mathfrak{X}_{\text {rig }}$ has cuspidal reduction if $\bar{P}$ is closed point of $\overline{\mathbb{X}}-\bar{X}$, and otherwise we say it has non-cuspidal reduction. We use a similar terminology for points of $\mathfrak{Y}$ rig.

4.2. Valuations on $\mathfrak{X}_{\text {rig }}$ and $\mathfrak{Y}_{\text {rig. }}$ Let $\mathbb{C}_{p}$ be the completion of an algebraic closure of $\mathbb{Q}_{p}$. It has a valuation val : $\mathbb{C}_{p} \rightarrow \mathbb{Q} \cup\{\infty\}$ normalized so that $\operatorname{val}(p)=1$. Define

$$
\nu(x)=\min \{\operatorname{val}(x), 1\} .
$$

Lemma 4.2.1. Let $P \in \mathfrak{X}_{\text {rig }}$ and $Q \in \mathfrak{Y}_{\text {rig }}$ be points of non-cuspidal reduction.

(1) Let $\left\{t_{\beta}\right\}_{\beta \in \mathbb{B}}$ and $\left\{t_{\beta}^{\prime}\right\}_{\beta \in \mathbb{B}}$ be two sets of parameters at $\bar{P}$ as in (2.5.1). For any $\beta \in \tau(\bar{P})$ there is $f_{\beta} \in \widehat{\mathcal{O}}_{X, \bar{P}}, \epsilon_{\beta} \in \widehat{\mathcal{O}}_{X, \bar{P}}^{\times}$such that $t_{\beta}^{\prime}=\epsilon_{\beta} t_{\beta}+p f_{\beta}$.

(2) Let $\left\{x_{\beta}\right\}_{\beta \in I(\bar{Q})},\left\{x_{\beta}^{\prime}\right\}_{\beta \in I(\bar{Q})}$ be parameters as in 2.4.3). For any $\beta \in I(\bar{Q})$, there is $g_{\beta} \in \widehat{\mathcal{O}}_{Y, \bar{Q}}, \delta_{\beta} \in \widehat{\mathcal{O}}_{Y, \bar{Q}}^{\times}$such that $x_{\beta}^{\prime}=\delta_{\beta} x_{\beta}+p g_{\beta}$.

Proof. We denote the reduction modulo $p$ of a parameter $t$ by $\bar{t}$. Let $\bar{P}=\operatorname{sp}(P) \in \bar{X}$ be defined over $k$. Reducing modulo $p$ we obtain isomorphisms $k \llbracket \bar{t}_{\beta} \rrbracket \cong \widehat{\mathcal{O}}_{\bar{X}}, \bar{P} \cong k \llbracket \bar{t}_{\beta}^{\prime} \rrbracket$, where for each $\beta$ the vanishing loci of $\bar{t}_{\beta}$ and $\bar{t}_{\beta}^{\prime}$ are both equal to $Z_{\beta} \cap \operatorname{Spf}\left(\widehat{\mathcal{O}}_{\bar{X}}, \bar{P}\right)$. This proves the claim. A similar proof works for the second part; by Lemma 2.4.2, the vanishing locus of $\bar{x}_{\beta}$ is $V_{\beta}^{+} \cap \operatorname{Spf}\left(\widehat{\mathcal{O}}_{\bar{Y}, \bar{Q}}\right)$.

We now define valuation vectors for points on $\mathfrak{X}_{\text {rig }}$ and $\mathfrak{Y}_{\text {rig. }}$. Let $P \in \mathfrak{X}_{\text {rig. }}$. Let $D_{P}=\mathrm{sp}^{-1}(\bar{P})$, which, by Berthelot's construction, is the rigid analytic space associated to $\operatorname{Spf}\left(\widehat{\mathcal{O}}_{X, \bar{P}}\right)$. If $P$ has non-cuspidal reduction, the parameters $t_{\beta}$ in (2.5.1) are functions on $D_{P}$. We define $\nu_{\mathfrak{X}}(P)=$ $\left(\nu_{\beta}(P)\right)_{\beta \in \mathbb{B}}$, where the entries $\nu_{\beta}(P)$ are given by

$$
\nu_{\beta}(P)= \begin{cases}\nu\left(t_{\beta}(P)\right) & \beta \in \tau(\bar{P}), \\ 0 & \beta \notin \tau(\bar{P}) .\end{cases}
$$

If $P$ has cuspidal reduction, this gives $\nu_{\beta}(P)=0$ for all $\beta \in \mathbb{B}$. By Lemma 4.2.1, the above definition is independent of the choice of parameters as in (2.5.1). In particular, we can define $\nu_{\mathfrak{X}}$ using parameters that are liftings of the partial Hasse invariants at the point $P$. More precisely, let $h_{\beta}$ be the partial Hasse invariant discussed in $\$ 2.5$. Then $\nu_{\beta}(P)=\nu\left(\tilde{h}_{\beta}(P)\right)$, where $\tilde{h}_{\beta}$ is any lift of $h_{\beta}$ locally at $P$.

Similarly, for $Q \in \mathfrak{Y}_{\text {rig }}$, we define $\nu_{\mathfrak{Y}}(Q)=\left(\nu_{\beta}(Q)\right)_{\beta \in \mathbb{B}}$, where

$$
\nu_{\beta}(Q)= \begin{cases}1 & \beta \in \eta(\bar{Q})-I(\bar{Q}), \\ \nu\left(x_{\beta}(Q)\right) & \beta \in I(\bar{Q}), \\ 0 & \beta \notin \eta(\bar{Q}) .\end{cases}
$$

Again, by Lemma 4.2.1, this definition is independent of the choice of parameters as in (2.4.3). For a point $Q \in \mathfrak{Y}_{\text {rig }}$ of cuspidal reduction, the above definition simplifies as follows: there is a unique $\mathfrak{t} \mid p$ such that $\bar{Q}$ is a $\mathfrak{t}$-cusp (see Definition 3.2.2), and

$$
\nu_{\beta}(Q)= \begin{cases}1 & \beta \in \mathbb{B}_{\mathfrak{t}^{*}}, \\ 0 & \beta \notin \mathbb{B}_{\mathfrak{t}^{*}} .\end{cases}
$$


Let $\mathbf{1}=(1)_{\beta \in \mathbb{B}}$ denote the constant vector of 1's, $\mathbf{0}=(0)_{\beta \in \mathbb{B}}$ the constant vector of 0's, etc.

Proposition 4.2.2. For any $Q \in \mathfrak{Y}_{\text {rig }}$, we have $\nu_{\mathfrak{Y}}(Q)+\nu_{\mathfrak{Y}}(w(Q))=\mathbf{1}$.

Proof. First we assume that $Q$, and hence $w(Q)$, have non-cuspidal reduction. Fix isomorphisms as in Lemma 2.7 .2 at $\bar{Q}$ and $w(\bar{Q})$. To avoid confusion, we will decorate any parameter with the point at which it is defined. For example, we will use $x_{\beta, \bar{Q}}$ to denote the parameter $x_{\beta}$ chosen at $\bar{Q}$. Assume that $\beta \in I(\bar{Q})=I(w(\bar{Q})$ ) (see Proposition 2.7.1). Then,

$$
\nu_{\beta}(Q)=\nu\left(x_{\beta, \bar{Q}}(Q)\right)=\nu\left(w^{*} y_{\beta, w(\bar{Q})}(Q)\right)=\nu\left(y_{\beta, w(\bar{Q})}(w(Q))\right) .
$$

Using the relation $x_{\beta, w(\bar{Q})} y_{\beta, w(\bar{Q})}=p$, we see that

$$
\nu_{\beta}(Q)=1-\nu\left(x_{\beta, w(\bar{Q})}(w(Q))\right)=1-\nu_{\beta}(w(Q)) .
$$

By definition, $0<\nu_{\beta}(Q)<1$ if and only if $\beta \in I(\bar{Q})$. Since $I(\bar{Q})=I(w(\bar{Q}))$, to prove the claim it remains to show that $\nu_{\beta}(Q)=1$ if and only if $\nu_{\beta}(w(Q))=0$. We have $\nu_{\beta}(Q)=1$ if and only if $\beta \in \eta(\bar{Q})-I(\bar{Q})=\ell(\varphi(w(\bar{Q})))-I(w(\bar{Q}))=\mathbb{B}-\eta(w(\bar{Q}))$. But this, by definition, is equivalent to $\nu_{\beta}(w(Q))=0$.

Now, assume $Q$ has cuspidal reduction. There is a unique $\mathfrak{t} \mid p$, such that $\bar{Q}$ is a $\mathfrak{t}$-cusp. By Proposition [3.2.3, $w(\bar{Q})$ is a $\mathfrak{t}^{*}$-cusp. The result now follows from the definition of $\nu_{\mathfrak{Y}}$.

4.3. The valuation cube. Let $\Theta=[0,1]^{\mathbb{B}}$ be the unit cube in $\mathbb{R}^{\mathbb{B}} \cong \mathbb{R}^{g}$. Its "open faces" can be encoded by vectors $\mathbf{a}=\left(a_{\beta}\right)_{\beta \in \mathbb{B}}$ such that $a_{\beta} \in\{0, *, 1\}$. The face corresponding to $\mathbf{a}$ is the set

$$
\mathcal{F}_{\mathbf{a}}:=\left\{\mathbf{v}=\left(v_{\beta}\right)_{\beta \in \mathbb{B}} \in \Theta: v_{\beta}=a_{\beta} \text { if } a_{\beta} \neq * \text {, and } 0<v_{\beta}<1 \text { otherwise }\right\} .
$$

There are $3^{g}$ such faces. The star of an open face $\mathcal{F}$ is $\operatorname{Star}(\mathcal{F})=\cup_{\overline{\mathcal{F}^{\prime}} \supseteq \mathcal{F}} \mathcal{F}^{\prime}$, where the union is over all open faces $\mathcal{F}^{\prime}$ whose topological closure contains $\mathcal{F}$. For a as above, we define

$$
\begin{aligned}
\eta(\mathbf{a}) & =\left\{\beta \in \mathbb{B}: a_{\beta} \neq 0\right\}, \\
I(\mathbf{a}) & =\left\{\beta \in \mathbb{B}: a_{\beta}=*\right\}, \\
\varphi(\mathbf{a}) & =r\left(\eta(\mathbf{a})^{c} \cup I(\mathbf{a})\right)=\left\{\beta \in \mathbb{B}: a_{\sigma^{-1} \circ \beta} \neq 1\right\} .
\end{aligned}
$$

Theorem 4.3.1. There is a one-to-one correspondence between the open faces of $\Theta$ and the strata $\left\{\mathbb{W}_{\varphi, \eta}\right\}$ of $\overline{\mathbb{Y}}$, given by

$$
\mathcal{F}_{\mathbf{a}} \mapsto \mathbb{W}_{\varphi(\mathbf{a}), \eta(\mathbf{a})}
$$

It has the following properties:

(1) $\nu_{\mathfrak{Y}}(Q) \in \mathcal{F}_{\mathbf{a}}$ if and only if $\bar{Q} \in \mathbb{W}_{\varphi(\mathbf{a}), \eta(\mathbf{a})}$.

(2) $\operatorname{dim}\left(\mathbb{W}_{\varphi(\mathbf{a}), \eta(\mathbf{a})}\right)=g-\operatorname{dim}\left(\mathcal{F}_{\mathbf{a}}\right)=\sharp\left\{\beta: a_{\beta} \neq *\right\}$.

(3) If $\mathcal{F}_{\mathbf{a}} \subseteq \overline{\mathcal{F}}_{\mathbf{b}}$, then $\mathbb{W}_{\varphi(\mathbf{b}), \eta(\mathbf{b}) \subseteq \overline{\mathbb{W}}_{\varphi(\mathbf{a}), \eta(\mathbf{a})}}$ and vice versa; that is, the correspondence is order reversing. In particular, $\nu_{\mathfrak{Y}}(Q) \in \operatorname{Star}\left(\mathcal{F}_{\mathbf{a}}\right) \Longleftrightarrow \bar{Q} \in \mathbb{Z}_{\varphi(\mathbf{a}), \eta(\mathbf{a})}$. 
Proof. (1) is clear from the definitions. To prove (2), we write

$$
g-\operatorname{dim}\left(\mathcal{F}_{\mathbf{a}}\right)=g-\sharp\left\{\beta \in \mathbb{B}: a_{\beta}=*\right\}=2 g-(\sharp \varphi(\mathbf{a})+\sharp \eta(\mathbf{a}))=\operatorname{dim}\left(\mathbb{W}_{\varphi(\mathbf{a}), \eta(\mathbf{a})}\right),
$$

using Theorem 3.2 .1 for the last equality.

Next, we prove (3). We have $\mathcal{F}_{\mathbf{a}} \subseteq \overline{\mathcal{F}}_{\mathbf{b}}$ if and only if the following hold:

$$
\begin{aligned}
& a_{\beta}=* \Rightarrow b_{\beta}=*, \\
& a_{\beta}=0 \Rightarrow b_{\beta} \neq 1, \\
& a_{\beta}=1 \Rightarrow b_{\beta} \neq 0 .
\end{aligned}
$$

These conditions, in turn, are equivalent to the following:

$$
\begin{aligned}
I(\mathbf{a}) & \subseteq I(\mathbf{b}), \\
r(\eta(\mathbf{a}))^{c} & \subseteq \varphi(\mathbf{b}), \\
\ell(\varphi(\mathbf{a}))^{c} & \subseteq \eta(\mathbf{b}) .
\end{aligned}
$$

The above conditions are equivalent to $(\varphi(\mathbf{b}), \eta(\mathbf{b})) \geq(\varphi(\mathbf{a}), \eta(\mathbf{a}))$, because we can write $\eta(\mathbf{a})=I(\mathbf{a}) \cup \ell(\varphi(\mathbf{a}))^{c} \subseteq I(\mathbf{b}) \cup \eta(\mathbf{b})=\eta(\mathbf{b})$, and similarly, $\varphi(\mathbf{a})=r(I(\mathbf{a})) \cup r(\varphi(\mathbf{a}))^{c} \subseteq$ $r(I(\mathbf{b})) \cup \varphi(\mathbf{b})=\varphi(\mathbf{b})$. The other direction of equivalence follows easily using the admissibility of all the pairs $(\varphi, \eta)$ appearing above. Finally, by Theorem 3.2.1 $(\varphi(\mathbf{b}), \eta(\mathbf{b})) \geq(\varphi(\mathbf{a}), \eta(\mathbf{a}))$ is equivalent to $\mathbb{W}_{\varphi(\mathbf{b}), \eta(\mathbf{b})} \subseteq \mathbb{Z}_{\varphi(\mathbf{a}), \eta(\mathbf{a})}=\overline{\mathbb{W}}_{\varphi(\mathbf{a}), \eta(\mathbf{a})}$. The proof is complete.

\section{The Canonical subgroup}

5.1. Some admissible open subsets of $\mathfrak{X}_{\text {rig }}$ and $\mathfrak{Y}_{\text {rig }}$. Let $K$ be a discretely valued complete subfield of $\mathbb{C}_{P}$ with uniformizer $\varpi$ and ring of integers $\mathcal{O}_{K}$. Let $\mathfrak{Z}$ be an admissible formal scheme over $\mathcal{O}_{K}$ and $\mathfrak{Z}_{\text {rig }}$ the rigid analytic space over $K$ associated to it à la Raynaud. Let $C$ be a closed subscheme of $\overline{\mathfrak{Z}}$, the special fibre of $\mathfrak{Z}$. Let $|\varpi| \leq \lambda \leq 1$ be an element of $p^{\mathbb{Q}}$.

One can define $[C]_{\leq \lambda}$, the closed tube of $C$ of radius $\lambda$, as in [5, 1.1.8]. It is a quasi-compact admissible open of $\mathfrak{Z}_{\text {rig }}$ defined as follows: if $C$ is defined by the vanishing of functions $f_{1}, \ldots, f_{m}$ in $\mathcal{O}(\overline{\mathfrak{Z}})$ with lifts $\tilde{f}_{1}, \ldots, \tilde{f}_{m}$ in $\mathcal{O}(\mathfrak{Z})$, then $[C]_{\leq \lambda}$ is defined by the inequalities $\left|\tilde{f}_{i}\right|_{\text {sup }} \leq \lambda$ for $1 \leq i \leq m$. Note that if $\lambda=1$ this gives the entire $\mathfrak{Z}_{\text {rig. }}$. Under the above assumptions on $\lambda$, this definition is independent of the choice of $f_{i}$ 's and their lifts. In the general case, $[C]_{\leq \lambda}$ can be constructed in the same way by using local generators for the ideal of $C$ and gluing these local constructions. The gluing is possible in view of the independence of the local construction of the set of generators of the ideal of $C$. This independence also implies the following: if $\bar{Q}$ is a closed point of $\overline{\mathfrak{Z}}$, then $\operatorname{sp}^{-1}(\bar{Q}) \cap[C]_{\leq \lambda}$ is the locus where $\left|\tilde{g}_{i}\right|_{\text {sup }} \leq \lambda$, where $\tilde{g}_{i}$ 's are any set of functions in $\mathcal{O}_{\mathfrak{Z}, \bar{Q}}$ whose reductions, $g_{i}$, define the closed subscheme $C \cap \operatorname{Spf}\left(\widehat{\mathcal{O}}_{\overline{\mathfrak{Z}}, \bar{Q}}\right)$.

If $C$ is a Cartier divisor on $\overline{\mathfrak{Z}}$, then one can similarly define $[C]_{\geq \lambda}$, which is a quasi-compact admissible open in $\mathfrak{Z}_{\text {rig: }}$ : write $C$ locally as the vanishing of a function $f$ which lifts to $\tilde{f} \in \mathcal{O}(\mathfrak{Z})$, and define $[C]_{\geq \lambda}$ locally by the inequality $|\tilde{f}|_{\text {sup }} \geq \lambda$. This will be independent of the choice of $f$ for $\lambda$ as above, and that allows gluing the local constructions. If $\bar{Q}$ is a closed point of $\overline{\mathfrak{Z}}$, then 
$\operatorname{sp}^{-1}(\bar{Q}) \cap[C]_{\geq \lambda}$ is the locus where $|\tilde{g}|_{\text {sup }} \geq \lambda$, where $\tilde{g}$ is any function in $\mathcal{O}_{\mathfrak{Z}, \bar{Q}}$ whose reduction defines the closed subscheme $C \cap \operatorname{Spf}\left(\widehat{\mathcal{O}}_{\overline{\mathfrak{Z}}}, \bar{Q}\right)$.

Lemma 5.1.1. Let $\beta \in \mathbb{B}$. Let $a \in[0,1] \cap \mathbb{Q}$.

(1) $\left[\mathbb{U}_{\sigma \circ \beta}^{+}\right]_{\leq(1 / p)^{1-a}}$ is a quasi-compact admissible open in $\mathfrak{Y}_{\text {rig }}$ whose points are

$$
\left\{Q \in \mathfrak{Y}_{\text {rig }}: \nu_{\beta}(Q) \leq a\right\} .
$$

(2) $\left[\mathbb{Z}_{\{\beta\}}\right]_{\geq(1 / p)^{a}}$ is a quasi-compact admissible open in $\mathfrak{X}_{\mathrm{rig}}$ whose points are

$$
\left\{P \in \mathfrak{X}_{\text {rig }}: \nu_{\beta}(P) \leq a\right\} .
$$

(3) Similarly, $\left[\mathbb{Z}_{\{\beta\}}\right]_{\leq(1 / p)^{a}}$ is a quasi-compact admissible open in $\mathfrak{X}_{\text {rig }}$ whose points are

$$
\left\{P \in \mathfrak{X}_{\text {rig }}: \nu_{\beta}(P) \geq a\right\} .
$$

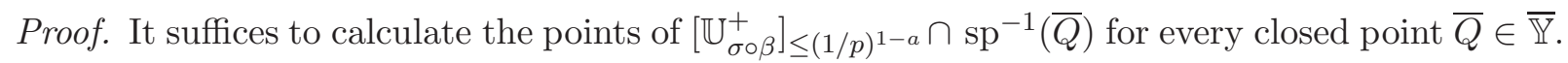
If $a=1$, then $\left[\mathbb{U}_{\sigma \circ \beta}^{+}\right]_{\leq(1 / p)^{1-a}}=\mathfrak{Y}_{\text {rig }}$ and the result follows. Assume $a<1$. Let $\bar{Q}$ be a closed point in $\overline{\mathbb{Y}}$. Then, by Lemma 3.2.4, $\mathbb{U}_{\sigma \circ \beta}^{+} \cap \operatorname{Spf}\left(\widehat{\mathcal{O}}_{\bar{Y}, \bar{Q}}\right)$ is given by the vanishing of

$$
\begin{cases}y_{\beta} & \beta \in I(\bar{Q}), \\ 1 & \beta \notin \ell(\varphi(\bar{Q})), \\ 0 & \beta \in \ell(\varphi(\bar{Q}))-\eta(\bar{Q}) .\end{cases}
$$

In the first case, $\mathrm{sp}^{-1}(\bar{Q}) \cap\left[\mathbb{U}_{\sigma \circ \beta}^{+}\right]_{\leq(1 / p)^{1-a}}$ is given by the inequality: $\left|y_{\beta}(Q)\right| \leq(1 / p)^{1-a}$, or, equivalently, $\nu_{\beta}(Q)=\nu\left(x_{\beta}(Q)\right) \leq a$. In the second case, $\mathrm{sp}^{-1}(\bar{Q}) \cap\left[\mathbb{U}_{\sigma \circ \beta}^{+}\right]_{\leq(1 / p)^{1-a}}$ is empty. The result follows, as in this case, $\beta \in \eta(\bar{Q})-I(\bar{Q})$, and hence, $\nu_{\beta}(Q)=1>a$. In the last case, $\mathrm{sp}^{-1}(\bar{Q}) \subseteq\left[\mathbb{U}_{\sigma \circ \beta}^{+}\right]_{\leq(1 / p)^{1-a}}$. The result again follows, as $\beta \notin \eta(\bar{Q})$ implies that $\nu_{\beta}(Q)=0 \leq a$.

Now we prove part (2). Again, the case $a=1$ is immediate, and we assume $a<1$. Let $\bar{P}$ be a closed point in $\overline{\mathbb{X}}$. The stratum $\mathbb{Z}_{\{\beta\}}$ is a divisor on $\overline{\mathbb{X}}$. In fact, $\mathbb{Z}_{\{\beta\}} \cap \operatorname{Spf}\left(\widehat{\mathcal{O}}_{\bar{X}}, \bar{P}\right)$ is given by the vanishing of

$$
\begin{cases}t_{\beta} & \beta \in \tau(\bar{P}), \\ 1 & \beta \notin \tau(\bar{P}) .\end{cases}
$$

In the first case, $\operatorname{sp}^{-1}(\bar{P}) \cap\left[\mathbb{Z}_{\{\beta\}}\right]_{\geq(1 / p)^{a}}$ is given by the inequality: $\left|t_{\beta}(P)\right| \geq(1 / p)^{a}$, or, equivalently, $\nu_{\beta}(P)=\nu\left(t_{\beta}(P)\right) \leq a$. In the second case, $\operatorname{sp}^{-1}(\bar{P}) \subseteq\left[\mathbb{Z}_{\{\beta\}}\right]_{\geq(1 / p)^{a}}$. The result follows, as in this case we always have $\nu_{\beta}(P)=0 \leq a$.

The remaining statement can be proved in the same way.

Corollary 5.1.2. Let $\mathbf{a}=\left(a_{\beta}\right)_{\beta \in \mathbb{B}}$ and $\mathbf{b}=\left(b_{\beta}\right)_{\beta \in \mathbb{B}}$ both belong to $\Theta \cap \mathbb{Q}^{\mathbb{B}}$. Assume that for each $\beta$, we have $a_{\beta} \leq b_{\beta}$. There is a quasi-compact admissible open $\mathfrak{Y}_{\text {rig }}[\mathbf{a}, \mathbf{b}]$ of $\mathfrak{Y}_{\text {rig }}$ whose points are

$$
\left\{Q \in \mathfrak{Y}_{\text {rig }}: a_{\beta} \leq \nu_{\beta}(Q) \leq b_{\beta}, \forall \beta \in \mathbb{B}\right\} .
$$

Similarly, there exits a quasi-compact admissible open $\mathfrak{X}_{\text {rig }}[\mathbf{a}, \mathbf{b}]$ of $\mathfrak{X}_{\text {rig }}$ whose points are

$$
\left\{P \in \mathfrak{X}_{\text {rig }}: a_{\beta} \leq \nu_{\beta}(P) \leq b_{\beta}, \forall \beta \in \mathbb{B}\right\} .
$$


Proof. Define

$$
\mathfrak{Y}_{\text {rig }}[\mathbf{a}, \mathbf{b}]=\bigcap_{\beta \in \mathbb{B}}\left[\mathbb{U}_{\sigma \circ \beta}^{+}\right]_{\leq(1 / p)^{1-b_{\beta}}} \bigcap \bigcap_{\beta \in \mathbb{B}} w\left(\left[\mathbb{U}_{\sigma \circ \beta}^{+}\right]_{\leq(1 / p)^{a_{\beta}}}\right) .
$$

This is a finite intersection of quasi-compact admissible opens in a quasi-separated rigid analytic space, and hence is a quasi-compact admissible open of $\mathfrak{Y}_{\text {rig }}$ with the desired property. Similarly, define

$$
\mathfrak{X}_{\text {rig }}[\mathbf{a}, \mathbf{b}]=\bigcap_{\beta \in \mathbb{B}}\left[\mathbb{Z}_{\{\beta\}}\right]_{\geq(1 / p)^{b_{\beta}}} \bigcap \bigcap_{\beta \in \mathbb{B}}\left[\mathbb{Z}_{\{\beta\}}\right]_{\leq(1 / p)^{a_{\beta}}},
$$

which is a quasi-compact admissible open of $\mathfrak{X}_{\text {rig }}$ with the desired property.

Proposition 5.1.3. Let $\Gamma$ be a subset of $\Theta$ with the property that if $\left(a_{\beta}\right)_{\beta \in \mathbb{B}} \in \Gamma$ and $b_{\beta} \leq a_{\beta}$ for all $\beta \in \mathbb{B}$, then $\left(b_{\beta}\right)_{\beta \in \mathbb{B}} \in \Gamma$.

(1) There is an admissible open subset $\mathfrak{Y}_{\text {rig }} \Gamma$ of $\mathfrak{Y}_{\text {rig }}$ whose points are $\left\{Q \in \mathfrak{Y}_{\text {rig }}: \nu_{\mathfrak{Y}}(Q) \in \Gamma\right\}$. The collection $\left\{\mathfrak{Y}_{\text {rig }}[\mathbf{0}, \mathbf{a}]: \mathbf{a} \in \Gamma\right\}$ is an admissible covering of $\mathfrak{Y}_{\mathrm{rig}} \Gamma$.

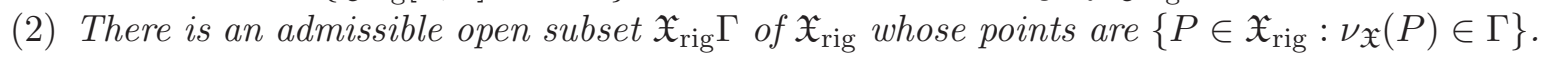
The collection $\left\{\mathfrak{X}_{\mathrm{rig}}[\mathbf{0}, \mathbf{a}]: \mathbf{a} \in \Gamma\right\}$ is an admissible covering of $\mathfrak{X}_{\mathrm{rig}} \Gamma$.

Proof. It is enough to show that the collection of quasi-compact opens $\left\{\mathfrak{Y}_{\text {rig }}[\mathbf{0}, \mathbf{a}]: \mathbf{a} \in \Gamma\right\}$ is an admissible covering: that is, for any affinoid algebra $A$, and any $f: \operatorname{Spm}(A) \rightarrow \mathfrak{Y}_{\text {rig }}$ whose image lies in the union of the subsets in this collection, the pull-back covering of $\operatorname{Spm}(A)$ has a finite sub-covering. This follows from a standard application of the maximum modulus principle. The second statement follows in the same way.

5.2. The section on the ordinary locus. By Corollary 5.1 .2 , we have the following admissible opens

$$
\begin{aligned}
& \mathfrak{X}_{\text {rig }}^{\circ}:=\mathfrak{X}_{\text {rig }}[\mathbf{0}, \mathbf{0}]=\left\{P \in \mathfrak{X}_{\text {rig }}: \nu_{\mathfrak{X}}(P)=\mathbf{0}\right\}, \\
& \mathfrak{Y}_{\text {rig }}^{\circ}:=\mathfrak{Y}_{\text {rig }}[\mathbf{0}, \mathbf{0}]=\left\{Q \in \mathfrak{Y}_{\text {rig }}: \nu_{\mathfrak{Y}}(Q)=\mathbf{0}\right\},
\end{aligned}
$$

of $\mathfrak{X}_{\text {rig }}$ and $\mathfrak{Y}_{\text {rig, }}$, respectively. By Theorem 4.3.1, and Definition 3.2.5, we have:

$$
\begin{aligned}
& \mathfrak{X}_{\text {rig }}^{\circ}=\operatorname{sp}^{-1}\left(\overline{\mathbb{X}}^{\text {ord }}\right)=\operatorname{sp}^{-1}\left(\mathbb{W}_{\emptyset}\right), \\
& \mathfrak{Y}_{\text {rig }}^{\circ \circ}=\operatorname{sp}^{-1}\left(\overline{\mathbb{Y}}_{F}^{\text {ord }}\right)=\operatorname{sp}^{-1}\left(\mathbb{W}_{\mathbb{B}, \emptyset}\right) .
\end{aligned}
$$

Let $\mathfrak{Y}_{\text {rig }}^{\circ}$ be $\mathrm{sp}^{-1}\left(\overline{\mathbb{Y}}^{\text {ord }}\right)=\pi^{-1}\left(\mathrm{sp}^{-1}\left(\overline{\mathbb{X}}^{\text {ord }}\right)\right)=\pi^{-1}\left(\mathfrak{X}_{\text {rig }}^{\circ}\right)$.

Proposition 5.2.1. There is a section,

$$
s^{\circ}: \mathfrak{X}_{\text {rig }}^{\circ} \rightarrow \mathfrak{Y}_{\text {rig }}^{\circ}
$$

to $\pi: \mathfrak{Y}_{\text {rig }}^{\circ} \rightarrow \mathfrak{X}_{\text {rig }}^{\circ}$, whose image is $\mathfrak{Y}_{\text {rig }}^{\circ}$.

Proof. Let $\mathfrak{X}^{\circ}$ be the open formal subscheme of $\mathfrak{X}$ supported on the ordinary locus; similarly, define $\mathfrak{Y}^{\circ}$. The special fibre of $\mathfrak{X}^{\circ}$ is $\overline{\mathbb{X}}^{\text {ord }}$ and its generic fibre is $\mathfrak{X}_{\text {rig }}^{\circ}$; similar results hold for $\mathfrak{Y}^{\circ}$. The morphism $\pi: \mathfrak{Y}^{\circ} \rightarrow \mathfrak{X}^{\circ}$ is proper and quasi-finite, and hence finite. We will show that $\left.\pi\right|_{\mathfrak{Y}_{\text {rig }}^{\circ \circ}}: \mathfrak{Y}_{\text {rig }}^{\circ} \rightarrow \mathfrak{X}_{\text {rig }}^{\circ}$ is an isomorphism. It is enough to prove this locally on the base. 
Let $U=\operatorname{Spf}(A)$ be an affine open formal subscheme of $\mathfrak{X}^{\circ}$. Let $\pi^{-1}(U)=\operatorname{Spf}(B) \subset \mathfrak{Y}^{\circ}$. The morphism $\pi$ induces a finite ring homomorphism $\pi^{*}: A \rightarrow B$. Since $\overline{\mathbb{Y}}_{F}^{\text {ord }}$ is a union of connected components of $\overline{\mathbb{Y}}^{\text {ord }}$, we have a decomposition $\overline{\mathbb{Y}}^{\text {ord }}=\overline{\mathbb{Y}}_{F}^{\text {ord }} \cup\left(\overline{\mathbb{Y}}^{\text {ord }}-\overline{\mathbb{Y}}_{F}^{\text {ord }}\right)$ leading to a decomposition

$$
B \otimes \kappa=e_{1}(B \otimes \kappa) \oplus e_{2}(B \otimes \kappa),
$$

where $e_{1}$ and $e_{2}$ are idempotents satisfying $e_{1}+e_{2}=1$, and $\operatorname{Spec}\left(e_{1}(B \otimes \kappa)\right)=\operatorname{Spec}(B \otimes \kappa) \cap \overline{\mathbb{Y}}_{F}^{\text {ord }}$. Using Hensel's lemma for the polynomial $x^{2}-x$, we can lift these idempotents to idempotents $\tilde{e}_{1}$ and $\tilde{e}_{2}$ in $B$. The composite homomorphism $A \otimes \kappa \rightarrow B \otimes \kappa \rightarrow e_{1}(B \otimes \kappa)$ is an isomorphism by the existence of the Kernel-of-Frobenius section. Therefore, the composite homomorphism $A \rightarrow B \rightarrow \tilde{e}_{1} B$ is a finite morphism whose reduction modulo $p$ is an isomorphism, and whose generic fibre is finite-flat (using an argument as in Lemma 7.1.1). Since both the generic fibre and special fibre of this map are flat, it follows that it is finite flat; having a reduction modulo $p$ which is an isomorphism, it follows that it is an isomorphism. Let $V=\operatorname{Spf}\left(\tilde{e}_{1} B\right)$. We have shown that $\left.\pi\right|_{V_{\text {rig }}}: V_{\text {rig }} \rightarrow U_{\text {rig }}$ is an isomorphism. To finish the proof, we need to prove that $V_{\text {rig }}=\pi^{1}\left(U_{\text {rig }}\right) \cap \mathfrak{Y}_{\text {rig. }}^{\circ \circ}$. But this is true, since $V_{\text {rig }}$ is the region in $\operatorname{Spf}(B)_{\text {rig }}=\pi^{-1}\left(U_{\text {rig }}\right)$ which specializes to $\operatorname{Spec}\left(e_{1}(B \otimes \kappa)\right)=\operatorname{Spec}(B \otimes \kappa) \cap \overline{\mathbb{Y}}_{F}^{\text {ord }}$.

5.3. The main theorem. Let $\Gamma=\left\{\mathbf{a} \in \Theta: a_{\beta}+p a_{\sigma^{-1} \circ \beta}<p\right.$ for all $\left.\beta \in \mathbb{B}\right\}$. Then, by Proposition 5.1.3, we have the following admissible open sets:

$$
\begin{aligned}
\mathcal{U} & :=\mathfrak{X}_{\text {rig }} \Gamma=\left\{P \in \mathfrak{X}_{\text {rig }}: \nu_{\beta}(P)+p \nu_{\sigma^{-1} \circ \beta}(P)<p, \forall \beta \in \mathbb{B}\right\}, \\
\mathcal{V} & :=\mathfrak{Y}_{\text {rig }} \Gamma=\left\{Q \in \mathfrak{Y}_{\text {rig }}: \nu_{\beta}(Q)+p \nu_{\sigma^{-1} \circ \beta}(Q)<p, \forall \beta \in \mathbb{B}\right\} .
\end{aligned}
$$

Recall that $\mathbb{B}=\operatorname{Emb}\left(L, \mathbb{Q}_{\kappa}\right)=\coprod_{\mathfrak{p}} \mathbb{B}_{\mathfrak{p}}$, where $\mathfrak{p}$ runs over prime ideals of $\mathcal{O}_{L}$ dividing $p$. For $\mathfrak{p} \mid p$, let

$$
\begin{aligned}
\mathcal{V}_{\mathfrak{p}}:=\left\{Q \in \mathfrak{Y}_{\text {rig }}: \nu_{\beta}(Q)+p \nu_{\sigma^{-1} \circ \beta}(Q)<p\right. & \left.\forall \beta \in \mathbb{B}_{\mathfrak{p}}\right\}, \\
\mathcal{W}_{\mathfrak{p}}:=\left\{Q \in \mathfrak{Y}_{\text {rig }}: \nu_{\beta}(Q)+p \nu_{\sigma^{-1} \circ \beta}(Q)>p\right. & \left.\forall \beta \in \mathbb{B}_{\mathfrak{p}}\right\} .
\end{aligned}
$$

By Proposition 5.1 .3 these are admissible open sets. Note that $\mathcal{V}=\cap_{\mathfrak{p} \mid p} \mathcal{V}_{\mathfrak{p}}$. Let

$$
\mathcal{W}:=\bigcup_{\emptyset \neq S \subseteq\{\mathfrak{p} \mid p\}}\left[\bigcap_{\mathfrak{p} \in S} \mathcal{W}_{\mathfrak{p}} \cap \bigcap_{\mathfrak{p} \notin S} \mathcal{V}_{\mathfrak{p}}\right] .
$$

We now prove our main theorem on the existence of canonical subgroups of abelian varieties with real multiplication.

Theorem 5.3.1 (The Canonical Subgroup Theorem). Let notation be as above.

(1) $\pi(\mathcal{V})=\mathcal{U}$.

(2) There is a section $s^{\dagger}: \mathcal{U} \rightarrow \mathcal{V}$ extending $s^{\circ}: \mathfrak{X}_{\text {rig }}^{\circ} \rightarrow \mathfrak{Y}_{\text {rig }}^{\circ}$.

Proof. Before we prove the theorem, we need a number of results. 
Lemma 5.3.2. Let $Q \in \mathfrak{Y}_{\text {rig }}, P=\pi(Q)$, and $\beta \in \mathbb{B}$. Then, $\beta \in \varphi(\bar{Q}) \cap \eta(\bar{Q})$ if and only if $\nu_{\beta}(Q) \neq 0$ and $\nu_{\sigma^{-1} \circ \beta}(Q) \neq 1$. In that case, $Q$ has non-cuspidal reduction; choose parameters $\left\{t_{\beta}\right\}_{\beta \in \mathbb{B}}$ at $\bar{P}$ as in (2.5.1), and parameters $\left\{x_{\beta}, y_{\beta}\right\}_{\beta \in I(\bar{Q})}$ at $\bar{Q}$ as in 2.4.2). We have:

$$
\pi^{*}\left(t_{\beta}\right) \underset{\bmod p \widehat{\mathcal{O}}_{Y, \bar{Q}}}{\equiv}\left\{\begin{array}{lll}
u x_{\beta}+v y_{\sigma^{-1} \circ \beta}^{p} & \text { if } \nu_{\beta}(Q) \neq 1, & \nu_{\sigma^{-1} \circ \beta}(Q) \neq 0 ; \\
u x_{\beta} & \text { if } \nu_{\beta}(Q) \neq 1, & \nu_{\sigma^{-1} \circ \beta}(Q)=0 ; \\
v y_{\sigma^{-1} \circ \beta}^{p} & \text { if } \nu_{\beta}(Q)=1, & \nu_{\sigma^{-1} \circ \beta}(Q) \neq 0 ; \\
0 & \text { if } \nu_{\beta}(Q)=1, & \nu_{\sigma^{-1} \circ \beta}(Q)=0 .
\end{array}\right.
$$

In the formulas above, $u, v$ are units in $\widehat{\mathcal{O}}_{Y, \bar{Q}}$. It follows that, respectively,

$$
\nu_{\beta}(P)=\left\{\begin{array}{l}
\nu_{\beta}\left(u x_{\beta}(Q)+v y_{\sigma^{-1} \circ \beta}^{p}(Q)\right) \\
\nu_{\beta}(Q) \\
p\left(1-\nu_{\sigma^{-1} \circ \beta}(Q)\right) \\
1 .
\end{array}\right.
$$

Proof. This follows immediately from the Key Lemma 2.8.1, The various cases are obtained by reinterpreting the conditions appearing in the lemma in terms of valuations, using directly the definition of valuations.

Remark 5.3.3. In Lemma 5.3.2, it automatically follows that $\left\{\beta, \sigma^{-1} \circ \beta\right\} \subseteq I(\bar{Q})$ in the first case, $\beta \in I(\bar{Q})$ in the second case, and $\sigma^{-1} \circ \beta \in I(\bar{Q})$ in the third case.

Lemma 5.3.4. Let $\mathfrak{p} \mid p$ and $\beta \in \mathbb{B}_{\mathfrak{p}}$. Let $Q \in \mathfrak{Y}_{\text {rig }}$, and $P=\pi(Q)$.

(1) If $Q \in \mathcal{V}_{\mathfrak{p}}$ then $\nu_{\beta}(P)=\nu_{\beta}(Q)$.

(2) If $Q \in \mathcal{W}_{\mathfrak{p}}$ then $\nu_{\beta}(P)=p\left(1-\nu_{\sigma^{-1} \circ \beta}(Q)\right)$.

Proof. First we deal with the case $Q \in \mathcal{V}_{\mathfrak{p}}$. Then, either $\nu_{\beta}(Q)=0$ or $0<\nu_{\beta}(Q)<1$. If $\nu_{\beta}(Q)=$ 0 , then, by definition, $\beta \notin \eta(\bar{Q})$. Since $Q \in \mathcal{V}_{\mathfrak{p}}$ and $\sigma^{-1} \circ \beta \in \mathbb{B}_{\mathfrak{p}}$, it follows that $\nu_{\sigma^{-1} \circ \beta}(Q) \neq 1$, and, by definition, $\sigma^{-1} \circ \beta \in(\eta(\bar{Q})-I(\bar{Q}))^{c}=\ell(\varphi(\bar{Q}))$. Therefore, $\beta \in \varphi(\bar{Q})-\eta(\bar{Q})$. Corollary 2.3 .4 tells us that $\beta \notin \tau(\bar{P})$, and hence, $\nu_{\beta}(P)=0=\nu_{\beta}(Q)$.

Now assume $0<\nu_{\beta}(Q)<1$, and so $Q$ has non-cuspidal reduction. Since $Q \in \mathcal{V}_{\mathfrak{p}}$, we have $\nu_{\sigma^{-1} \circ \beta}(Q) \neq 1$, and hence, by Lemma 5.3.2, $\beta \in \varphi(\bar{Q}) \cap \eta(\bar{Q})$. There are two cases to consider:

- $\nu_{\sigma^{-1} \circ \beta}(Q)=0$. In this case, Lemma 5.3 .2 implies that $\nu_{\beta}(P)=\nu_{\beta}(Q)$.

- $\nu_{\sigma^{-1} \circ \beta}(Q) \neq 0$. In this case, by Lemma 5.3.2, we have

$$
\nu_{\beta}(P)=\nu\left(u x_{\beta}(Q)+v y_{\sigma^{-1} \circ \beta}^{p}(Q)\right) .
$$

Since $Q \in \mathcal{V}_{\mathfrak{p}}$, we have $\nu_{\beta}(Q)<p\left(1-\nu_{\sigma^{-1} \circ \beta}(Q)\right)$ and hence, by Remark 5.3.3, we have $\nu\left(u x_{\beta}(Q)\right)<\nu\left(v y_{\sigma^{-1} \circ \beta}^{p}(Q)\right)$. It follows that $\nu_{\beta}(P)=\nu\left(u x_{\beta}(Q)\right)=\nu_{\beta}(Q)$.

Now we deal with the case $Q \in \mathcal{W}_{\mathfrak{p}}$. It follows that $\nu_{\beta}(Q)>0$ for any $\beta \in \mathbb{B}_{\mathfrak{p}}$. Hence, either $\nu_{\sigma^{-1} \circ \beta}(Q)=1$ or $0<\nu_{\sigma^{-1} \circ \beta}(Q)<1$. If $\nu_{\sigma^{-1} \circ \beta}(Q)=1$, then $\sigma^{-1} \circ \beta \in \eta(\bar{Q})-I(\bar{Q})=\ell(\varphi(\bar{Q}))^{c}$, and since $\nu_{\beta}(Q)>0, \beta \in \eta(\bar{Q})$. It follows that $\beta \in \eta(\bar{Q})-\varphi(\bar{Q})$ and hence, by Corollary 2.3.4, $\beta \notin \tau(\bar{P})$. So, $\nu_{\beta}(P)=0=p\left(1-\nu_{\sigma^{-1} \circ \beta}(Q)\right)$ as desired.

Now suppose $0<\nu_{\sigma^{-1} \circ \beta}(Q)<1$, and, in particular, that $Q$ has non-cuspidal reduction. There are two cases: 
- $\nu_{\beta}(Q)=1$. Then, by Lemma 5.3.2, we have $\nu_{\beta}(P)=p\left(1-\nu_{\sigma^{-1} \circ \beta}(Q)\right)$.

- $\nu_{\beta}(Q) \neq 1$. In this case, by Lemma 5.3.2, we have

$$
\nu_{\beta}(P)=\nu\left(u x_{\beta}(Q)+v y_{\sigma^{-1} \circ \beta}^{p}(Q)\right) .
$$

Since $Q \in \mathcal{W}_{\mathfrak{p}}$, we have $\nu_{\beta}(Q)>p\left(1-\nu_{\sigma^{-1} \circ \beta}(Q)\right)$ and hence, by Remark 5.3.3, we have $\nu\left(u x_{\beta}(Q)\right)>\nu\left(v y_{\sigma^{-1} \circ \beta}^{p}(Q)\right)$. It follows that $\nu_{\beta}(P)=\nu\left(v y_{\sigma^{-1} \circ \beta}^{p}(Q)\right)=p\left(1-\nu_{\sigma^{-1} \circ \beta}(Q)\right)$.

Corollary 5.3.5. $\pi^{-1}(\mathcal{U}) \supseteq \mathcal{V} \cup \mathcal{W}$.

Lemma 5.3.6. Let $\beta \in \mathbb{B}, Q \in \mathfrak{Y}_{\text {rig }}$, and $P=\pi(Q)$. Suppose

$$
\begin{aligned}
& \nu_{\beta}(Q)+p \nu_{\sigma^{-1} \circ \beta}(Q) \leq p, \\
& \nu_{\sigma \circ \beta}(Q)+p \nu_{\beta}(Q) \geq p .
\end{aligned}
$$

Then, $P \notin \mathcal{U}$.

Proof. The conditions imply that $\nu_{\sigma^{-1} \circ \beta}(Q) \neq 1, \nu_{\beta}(Q) \neq 0$, and, in particular, $Q$ has noncuspidal reduction. Therefore, by Lemma 5.3.2, $\beta \in \varphi(\bar{Q}) \cap \eta(\bar{Q})$. We distinguish the four cases as in Lemma 5.3.2, and the Key Lemma.

Case $\mathbf{A}: \nu_{\beta}(Q) \neq 1$ and $\nu_{\sigma^{-1} \circ \beta}(Q) \neq 0$. In this case, $\sigma \circ \beta \in \varphi(\bar{Q}), \sigma^{-1} \circ \beta \in \eta(\bar{Q})$, and $\nu_{\beta}(P)=\nu\left(u x_{\beta}(Q)+v y_{\sigma^{-1} \circ \beta}^{p}(Q)\right)$ for some units $u, v \in \widehat{\mathcal{O}}_{Y, \bar{Q}}$. Also, by Remark 5.3.3, we have $\left\{\beta, \sigma^{-1} \circ \beta\right\} \subseteq I(\bar{Q})$. Hence, Equation $(\dagger)$ can be rephrased as $\nu\left(u x_{\beta}(Q)\right) \leq \nu\left(v y_{\sigma^{-1} \circ \beta}^{p}(Q)\right)$. It follows that, in this case, $\nu_{\beta}(P) \geq \nu\left(u x_{\beta}(Q)\right)=\nu_{\beta}(Q)$. Since $\nu_{\beta}(Q) \neq 1$, Equation (††) implies that $\nu_{\sigma \circ \beta}(Q)>0$, and hence, $\sigma \circ \beta \in \eta(\bar{Q})$. Since also $\sigma \circ \beta \in \varphi(\bar{Q})$, we can apply Lemma 5.3 .2 at $\sigma \circ \beta$. There are two cases to consider:

- Case A.I: $\nu_{\sigma \circ \beta}(Q)=1, \nu_{\beta}(Q) \neq 0$. In this case, we have $\nu_{\sigma \circ \beta}(P)=p\left(1-\nu_{\beta}(Q)\right)$. Therefore,

$$
\nu_{\sigma \circ \beta}(P)+p \nu_{\beta}(P) \geq p\left(1-\nu_{\beta}(Q)\right)+p \nu_{\beta}(Q)=p,
$$

and hence, $P \notin \mathcal{U}$.

- Case A.II: $\nu_{\sigma \circ \beta}(Q) \neq 1, \nu_{\beta}(Q) \neq 0$. By Remark 5.3.3, in this case we have $\sigma \circ \beta \in I(\bar{Q})$. We also know that $\nu_{\sigma \circ \beta}(P)=\nu\left(u^{\prime} x_{\sigma \circ \beta}(Q)+v^{\prime} y_{\beta}^{p}(Q)\right)$ for some units $u^{\prime}, v^{\prime} \in \widehat{\mathcal{O}}_{Y, \bar{Q}}$. Equation (††) is equivalent to $\nu\left(u^{\prime} x_{\sigma \circ \beta}(Q)\right) \geq \nu\left(v^{\prime} y_{\beta}^{p}(Q)\right)$. It follows that $\nu_{\sigma \circ \beta}(P) \geq$ $\nu\left(v^{\prime} y_{\beta}^{p}(Q)\right)=p \nu\left(y_{\beta}(Q)\right)$. Therefore,

$$
\nu_{\sigma \circ \beta}(P)+p \nu_{\beta}(P) \geq p \nu\left(y_{\beta}(Q)\right)+p \nu_{\beta}(Q)=p \nu\left(y_{\beta}(Q)\right)+p \nu\left(x_{\beta}(Q)\right)=p,
$$

and hence, $P \notin \mathcal{U}$.

Case B: $\nu_{\beta}(Q) \neq 1$ and $\nu_{\sigma^{-1} \circ \beta}(Q)=0$. In this case, $\sigma \circ \beta \in \varphi(\bar{Q})$, and $\sigma^{-1} \circ \beta \notin \eta(\bar{Q})$, and $\nu_{\beta}(P)=\nu_{\beta}(Q)$. Equation $(\dagger \dagger)$ implies that $\nu_{\beta}(Q) \neq 0$. By $\operatorname{Remark} 5.3 .3$, we have $\beta \in I(\bar{Q})$. Exactly as in Case A, we deduce that $\sigma \circ \beta \in \varphi(\bar{Q}) \cap \eta(\bar{Q})$. Applying Lemma 5.3 .2 at $\sigma \circ \beta$ we consider two cases: 
- Case B.I: $\nu_{\sigma \circ \beta}(Q)=1, \nu_{\beta}(Q) \neq 0$. In this case, we have $\nu_{\sigma \circ \beta}(P)=p\left(1-\nu_{\beta}(Q)\right)$. Therefore,

$$
\nu_{\sigma \circ \beta}(P)+p \nu_{\beta}(P)=p\left(1-\nu_{\beta}(Q)\right)+p \nu_{\beta}(Q)=p,
$$

and hence, $P \notin \mathcal{U}$.

- Case B.II: $\nu_{\sigma \circ \beta}(Q) \neq 1, \nu_{\beta}(Q) \neq 0$. In this case, $\nu_{\sigma \circ \beta}(P)=\nu\left(u^{\prime} x_{\sigma \circ \beta}(Q)+v^{\prime} y_{\beta}^{p}(Q)\right)$ for some units $u^{\prime}, v^{\prime} \in \widehat{\mathcal{O}}_{Y, \bar{Q}}$. By Remark 5.3.3, we have $\sigma \circ \beta \in I(\bar{Q})$. This implies that Equation ( $\dagger \dagger)$ is equivalent to $\nu\left(u^{\prime} x_{\sigma \circ \beta}(Q)\right) \geq \nu\left(v^{\prime} y_{\beta}^{p}(Q)\right)$. It follows that $\nu_{\sigma \circ \beta}(P) \geq$ $\nu\left(v^{\prime} y_{\beta}^{p}(Q)\right)=p \nu\left(y_{\beta}(Q)\right)$. Therefore

$$
\nu_{\sigma \circ \beta}(P)+p \nu_{\beta}(P) \geq p \nu\left(y_{\beta}(Q)\right)+p \nu_{\beta}(Q)=p \nu\left(y_{\beta}(Q)\right)+p \nu\left(x_{\beta}(Q)\right)=p,
$$

and hence, $P \notin \mathcal{U}$.

Case C: $\nu_{\beta}(Q)=1, \nu_{\sigma^{-1} \circ \beta}(Q) \neq 0$. By Remark 5.3.3, we have $\sigma^{-1} \circ \beta \in I(\bar{Q})$. In this case, $\nu_{\beta}(P)=p\left(1-\nu_{\sigma^{-1} \circ \beta}(Q)\right)$. Since $\nu_{\beta}(Q)=1$, Equation $(\dagger)$ implies that $\nu_{\sigma^{-1} \circ \beta}(Q) \leq(p-1) / p$. Hence,

$$
\nu_{\beta}(P)=p\left(1-\nu_{\sigma^{-1} \circ \beta}(Q)\right) \geq p(1-(p-1) / p)=1,
$$

which implies that $\nu_{\sigma \circ \beta}(P)+p \nu_{\beta}(P) \geq p$, and $P \notin \mathcal{U}$.

Case D: $\nu_{\beta}(Q)=1, \nu_{\sigma^{-1} \circ \beta}(Q)=0$. In this case, $\nu_{\beta}(P)=1$, hence, $\nu_{\sigma \circ \beta}(P)+p \nu_{\beta}(P) \geq p$ and $P \notin \mathcal{U}$.

Corollary 5.3.7. $\pi^{-1}(\mathcal{U})=\mathcal{V} \cup \mathcal{W}$.

Proof. Let $Q \in \mathfrak{Y}_{\text {rig }}-(\mathcal{V} \cup \mathcal{W})$. For simplicity, we define $\lambda_{\beta}=\nu_{\beta}(Q)+p \nu_{\sigma^{-1} \circ \beta}(Q)$. By definition, there is $\mathfrak{p} \mid p$ such that $Q \notin \mathcal{V}_{\mathfrak{p}} \cup \mathcal{W}_{\mathfrak{p}}$. Since $Q \notin \mathcal{W}_{\mathfrak{p}}$, there exists $\gamma \in \mathbb{B}_{\mathfrak{p}}$, such that $\lambda_{\gamma} \leq p$. Since $Q \notin \mathcal{V}_{\mathfrak{p}}$, there exists $i \geq 1$, such that $\lambda_{\sigma^{i} \circ \gamma} \geq p$. Let $i$ be the minimal positive integer with this property. Let $\beta=\sigma^{i-1} \circ \gamma$. If $i=1$, then $\lambda_{\beta} \leq p$ and $\lambda_{\sigma \circ \beta} \geq p$. If $i>1$, then by minimality of $i$, we find that $\lambda_{\beta}<p$ and $\lambda_{\sigma \circ \beta} \geq p$. At any rate, Equations $(\dagger)$ and $(\dagger \dagger)$ of Lemma 5.3.6 hold for $Q$, and so $\pi(Q) \notin \mathcal{U}$.

Let

$$
\Gamma=\left\{\mathbf{a} \in \Theta \cap \mathbb{Q}^{\mathbb{B}}: a_{\beta}+p a_{\sigma^{-1} \circ \beta}<p \text { for all } \beta \in \mathbb{B}\right\} .
$$

For $\mathbf{a} \in \Gamma$ and $S \subseteq\{\mathfrak{p} \mid p\}$, let $\mathbb{I}_{\mathbf{a}}^{S}=[\mathbf{c}, \mathbf{d}]$, where $\mathbf{c}=\left(c_{\beta}\right), \mathbf{d}=\left(d_{\beta}\right)$ and

$$
\left[c_{\beta}, d_{\beta}\right]= \begin{cases}{\left[1-\frac{1}{p} \cdot a_{\sigma \circ \beta}, 1\right]} & \beta \in \mathbb{B}_{\mathfrak{p}}, \mathfrak{p} \in S, \\ {\left[0, a_{\beta}\right]} & \beta \in \mathbb{B}_{\mathfrak{p}}, \mathfrak{p} \notin S .\end{cases}
$$

By Corollary [5.1.2, $\mathfrak{Y}$ rig $\mathbb{I}_{\mathbf{a}}^{S}$ is a quasi-compact admissible open of $\mathfrak{Y}$ rig.

Corollary 5.3.8. Let notation be as above. We have

$$
\pi^{-1}\left(\mathfrak{X}_{\text {rig }}[\mathbf{0}, \mathbf{a}]\right)=\mathfrak{Y}_{\text {rig }}[\mathbf{0}, \mathbf{a}] \cup \bigcup_{\emptyset \neq S \subseteq\{\mathfrak{p} \mid p\}} \mathfrak{Y}_{\text {rig }} \mathbb{I}_{\mathbf{a}}^{S} .
$$

Proof. This follows from Corollary 5.3.7 and Lemma 5.3.4. 
We continue with the proof of Theorem 5.3.1. Let $\mathbf{a} \in \Gamma$. For simplicity, we denote by $\mathcal{R}$ the quasi-compact admissible open $\cup_{\emptyset \neq S \subseteq\{\mathfrak{p} \mid p\}} \mathfrak{Y}_{\text {rig }} \mathbb{I}_{\mathbf{a}}^{S}$. By Corollary 5.3.8, the morphism

$$
\pi: \mathfrak{Y}_{\text {rig }}[\mathbf{0}, \mathbf{a}] \cup \mathcal{R} \rightarrow \mathfrak{X}_{\text {rig }}[\mathbf{0}, \mathbf{a}]
$$

is finite and flat of the same degree as that of $\pi: \mathfrak{Y}_{\text {rig }} \rightarrow \mathfrak{X}_{\text {rig }}$, that is, $\prod_{\mathfrak{p} \mid p}\left(p^{f(\mathfrak{p})}+1\right)$. From the definition, we see that $\mathfrak{Y}_{\text {rig }}[\mathbf{0}, \mathbf{a}] \cap \mathcal{R}=\emptyset$, and since both $\mathfrak{Y}_{\text {rig }}[\mathbf{0}, \mathbf{a}]$ and $\mathcal{R}$ are quasi-compact, and $\mathfrak{Y}_{\text {rig }}$ is quasi-separated, we see that they provide an admissible disjoint covering of $\pi^{-1}\left(\mathfrak{X}_{\text {rig }}[\mathbf{0}, \mathbf{a}]\right)$. In particular, $\pi: \mathfrak{Y}_{\text {rig }}[\mathbf{0}, \mathbf{a}] \rightarrow \mathfrak{X}_{\text {rig }}[\mathbf{0}, \mathbf{a}]$ is a finite-flat morphism. We need a lemma.

Lemma 5.3.9. The morphism $\pi: \mathfrak{Y}_{\text {rig }}[\mathbf{0}, \mathbf{a}] \rightarrow \mathfrak{X}_{\text {rig }}[\mathbf{0}, \mathbf{a}]$ is an isomorphism.

Proof. Since $\pi: \mathfrak{Y}_{\text {rig }}[\mathbf{0}, \mathbf{a}] \rightarrow \mathfrak{X}_{\text {rig }}[\mathbf{0}, \mathbf{a}]$ is finite-flat, to prove the lemma it is enough to show that it has constant degree 1 . It is enough to calculate the degree of this morphism over an admissible open inside every connected component of $\mathfrak{X}_{\text {rig }}[\mathbf{0}, \mathbf{a}]$. We use $\mathfrak{X}_{\text {rig }}^{\circ}=\mathfrak{X}_{\text {rig }}[\mathbf{0}, \mathbf{0}] \subset$ $\mathfrak{X}_{\text {rig }}[\mathbf{0}, \mathbf{a}]$, which intersects every connected component of $\mathfrak{X}_{\text {rig }}[\mathbf{0}, \mathbf{a}]$, and whose inverse image under $\pi$ inside $\mathfrak{Y}_{\text {rig }}[\mathbf{0}, \mathbf{a}]$ is $\mathfrak{Y}_{\text {rig }}^{\circ}=\mathfrak{Y}_{\text {rig }}[\mathbf{0}, \mathbf{0}]$ by Corollary 5.3.8, By Proposition 5.2.1, the morphism $\pi: \mathfrak{Y}_{\text {rig }}^{\circ \circ} \rightarrow \mathfrak{X}_{\text {rig }}^{\circ}$ is an isomorphism and hence has degree 1. This proves the lemma.

By Lemma 5.3.9, for any a $\in \Gamma$ there is a section $s^{\mathbf{a}}: \mathfrak{X}_{\text {rig }}[\mathbf{0}, \mathbf{a}] \rightarrow \mathcal{V}$ to $\pi$ whose image is $\mathfrak{Y}_{\text {rig }}[\mathbf{0}, \mathbf{a}]$ and which extends $s^{\circ}=s^{\mathbf{0}}$. Furthermore, the sections $\left\{s^{\mathbf{a}}\right\}$ are compatible on intersections. Since by Proposition 5.1 .3 the collection $\left\{\mathfrak{X}_{\text {rig }}[\mathbf{0}, \mathbf{a}]\right\} \mathbf{a} \in \Gamma$ admissibly covers $\mathcal{U}$, we conclude that there is a section

$$
s^{\dagger}: \mathcal{U} \rightarrow \mathcal{V}
$$

to $\pi$ which extends $s^{\circ}$. This completes the proof of Theorem 5.3.1.

\subsection{Properties of the canonical subgroup.}

Definition 5.4.1. Let $K \supseteq W(\kappa)$ be a completely valued field. Let $\underline{A}$ be an abelian variety over

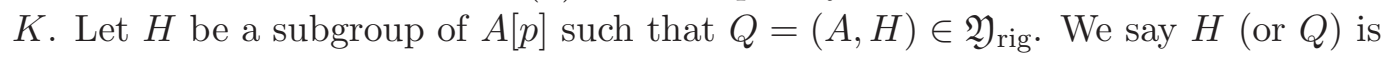

- canonical at $\mathfrak{p}$, if $Q \in \mathcal{V}_{\mathfrak{p}}$; that is, if for all $\beta \in \mathbb{B}_{\mathfrak{p}}$, we have $\nu_{\beta}(Q)+p \nu_{\sigma^{-1} \circ \beta}(Q)<p$;

- anti-canonical at $\mathfrak{p}$, if $Q \in \mathcal{W}_{\mathfrak{p}}$; that is, for all $\beta \in \mathbb{B}_{\mathfrak{p}}$, we have $\nu_{\beta}(Q)+p \nu_{\sigma^{-1} \circ \beta}(Q)>p$;

- too singular at $\mathfrak{p}$, if it is neither canonical nor anti-canonical at $\mathfrak{p}$;

- canonical, if it is canonical at all $\mathfrak{p}$ dividing $p$. This is equivalent to $Q$ belonging to $\mathcal{V}$. Such an $H$ is called the canonical subgroup of $\underline{A}$.

- anti-canonical, if it is anti-canonical at all $\mathfrak{p}$ dividing $p$;

- too singular, if it is neither canonical nor anti-canonical; equivalently, if it is too singular at some $\mathfrak{p}$ dividing $p$.

We study the reduction properties of the canonical subgroup.

Theorem 5.4.2. Let $K \supseteq W(\kappa)$ be a completely valued field. Let $\underline{A} / K$ be an abelian variety corresponding to a point of non-cuspidal reduction $P \in \mathcal{U}$, and hence

$$
\nu_{\beta}(\underline{A})+p \nu_{\sigma^{-1} \circ \beta}(\underline{A})<p \quad \forall \beta \in \mathbb{B} .
$$

Enlarging $K$, we can assume that there exists $r$ in $K$ such that $\operatorname{val}(r)=\max \left\{\nu_{\beta}(\underline{A}): \beta \in \mathbb{B}\right\}$, and that the canonical subgroup of $A$ is defined over $K$. 
(1) The canonical subgroup of $\underline{A}$ reduces to $\operatorname{Ker}(\mathrm{Fr})$ modulo $p / r$.

(2) Assume $K$ contains $r^{1 / p}$, a p-th root of $r$. Let $C$ be an anti-canonical subgroup of $\underline{A}$. Then, $C$ reduces to $\operatorname{Ker}($ Ver $)$ modulo $p / r^{1 / p}$.

Proof. Let $\underline{A}$ correspond to $P \in \mathcal{U}$. Let $H$ be the canonical subgroup of $\underline{A}$, and $Q=(\underline{A}, H)$. Since $Q \in \mathcal{V}$, we have $\nu_{\beta}(Q) \neq 1$ for all $\beta \in \mathbb{B}$. It follows from the definition of valuations that $\eta(\bar{Q})=I(\bar{Q})$. Since $I(\bar{Q})=\ell(\varphi(\bar{Q})) \cap \eta(\bar{Q})$, we find that $\eta(\bar{Q}) \subseteq \ell(\varphi(\bar{Q}))$. The admissibility condition $\ell(\varphi(\bar{Q}))^{c} \subseteq \eta(\bar{Q})$ then implies that $\varphi(\bar{Q})=\mathbb{B}$. This shows that $\bar{Q}$ belongs to $Z_{\mathbb{B}, \emptyset}=$ $\bar{Y}_{F}=s(\bar{X})$. Since $s$ is a section to $\pi: \bar{Y} \rightarrow \bar{X}$ and $\pi(\bar{Q})=\bar{P}$, it follows that $s(\bar{P})=\bar{Q}$.

Using Lemma 2.7.3, choose isomorphisms as in (2.4.3) and (2.5.1) such that (thinking of variables modulo $p$ ) we have $s^{*}\left(x_{\beta}\right)=t_{\beta}$ and $s^{*}\left(y_{\beta}\right)=0$ for all $\beta \in I(\bar{Q})$, and $s^{*}\left(z_{\gamma}\right)=t_{\gamma}$ for all $\gamma \in I(\bar{Q})^{c}$.

We have a commutative diagram, extending the relation $\pi(Q)=P$,

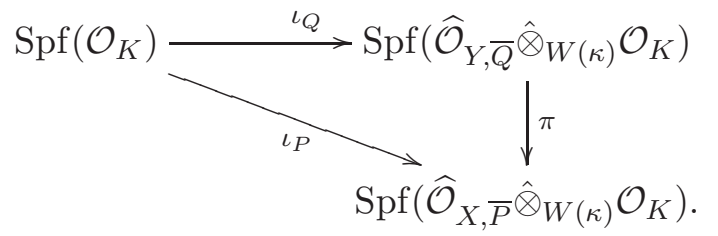

Let $\mathfrak{m}$ be the ideal of $\mathcal{O}_{K}$ generated by all $y_{\beta}(Q)$ for $\beta \in I(\bar{Q})$. Then $\mathfrak{m}=(p / r)$. We will denote reductions of $\iota_{Q}$ and $\iota_{P}$ modulo $\mathfrak{m}$, respectively, by $\bar{\iota}_{Q}$ and $\bar{\iota}_{P}$. Let $\bar{\pi}$ and $\bar{s}$ denote, respectively, the base extension from $\kappa$ to $\mathcal{O}_{K} / \mathfrak{m}$ of the natural projection $\pi: \operatorname{Spf}\left(\widehat{\mathcal{O}}_{\bar{Y}}, \bar{Q}\right) \rightarrow \operatorname{Spf}\left(\widehat{\mathcal{O}}_{\bar{X}}, \bar{P}\right)$, and the Kernel-of-Frobenius section $s: \operatorname{Spf}\left(\widehat{\mathcal{O}}_{\bar{X}}, \bar{P}\right) \rightarrow \operatorname{Spf}\left(\widehat{\mathcal{O}}_{\bar{Y}}, \bar{Q}\right)$. We need to show $\bar{s} \circ \bar{\iota}_{P}=\bar{\iota}_{Q}$. For $f \in \widehat{\mathcal{O}}_{\bar{Y}, \bar{Q}} \otimes_{\kappa} \mathcal{O}_{K} / \mathfrak{m}$ we have

$$
\bar{\iota}_{P}^{*} \circ \bar{s}^{*}(f)-\bar{\iota}_{Q}^{*}(f)=\bar{\iota}_{Q}^{*} \circ \bar{\pi}^{*} \circ \bar{s}^{*}(f)-\bar{\iota}_{Q}^{*}(f)=\bar{\iota}_{Q}^{*}\left(\bar{\pi}^{*} \circ \bar{s}^{*}(f)-f\right),
$$

which we want to show is zero. Since $\bar{s}$ is a section to $\bar{\pi}$, we see that $\bar{\pi}^{*} \circ \bar{s}^{*}(f)-f$ is in the kernel of $\bar{s}^{*}$. By our choice of variables, this kernel is the ideal generated by all the $y_{\beta}$ for $\beta \in I(\bar{Q})$. So it is enough to show that $\tau_{Q}^{*}\left(y_{\beta}\right)=0$ for all $\beta \in I(\bar{Q})$. But $\tau_{Q}^{*}\left(y_{\beta}\right)$ is the reduction modulo $\mathfrak{m}$ of $y_{\beta}(Q)$, which is zero, as $\mathfrak{m}$ contains $y_{\beta}(Q)$ by definition.

We now prove a result which explains the geometry of the Hecke correpondence $U_{p}$ on the not-too-singular locus of $\mathfrak{Y}_{\text {rig. }}$ In the Appendix, we will describe how to generalize this result so that it would explain the geometry of the partial $U$-operators, $\left\{U_{\mathfrak{p}}\right\}$, on the not-too-singular locus.

Theorem 5.4.3. Let $K \supseteq W(\kappa)$ be a completely valued field. Let $\underline{A} / K$ be an abelian variety corresponding to a point on $\mathfrak{X}_{\text {rig. }}$. Let $H$ be a subgroup of $\underline{A}$ such that $(\underline{A}, H) \in \mathfrak{Y}_{\text {rig. }}$. Assume that $H$ is canonical at $\mathfrak{p}$.

(1) If $\nu_{\beta}(\underline{A})+p \nu_{\sigma^{-1} \circ \beta}(\underline{A})<1$ for all $\beta \in \mathbb{B}_{\mathfrak{p}}$, then $\nu_{\beta}(\underline{A} / H)=p \nu_{\sigma^{-1} \circ \beta}(\underline{A})$ for all $\beta \in \mathbb{B}_{\mathfrak{p}}$ and $A[p] / H$ is anti-canonical at $\mathfrak{p}$. In particular, if $H$ is canonical and $\nu_{\beta}(\underline{A})+p \nu_{\sigma^{-1} \circ \beta}(\underline{A})<1$ for all $\beta \in \mathbb{B}$, then $\nu_{\beta}(\underline{A} / H)=p \nu_{\sigma^{-1} \circ \beta}(\underline{A})$ for all $\beta \in \mathbb{B}$ and $A[p] / H$ is anti-canonical.

(2) If $1<\nu_{\beta}(\underline{A})+p \nu_{\sigma^{-1} \circ \beta}(\underline{A})<p$ for all $\beta \in \mathbb{B}_{\mathfrak{p}}$, then $\nu_{\beta}(\underline{A} / H)=1-\nu_{\beta}(\underline{A})$ for all $\beta \in \mathbb{B}_{\mathfrak{p}}$ and $A[p] / H$ is canonical at $\mathfrak{p}$. In particular, if $H$ is the canonical subgroup and if 
$1<\nu_{\beta}(\underline{A})+p \nu_{\sigma^{-1} \circ \beta}(\underline{A})<p$ for all $\beta \in \mathbb{B}$, then $\nu_{\beta}(\underline{A} / H)=1-\nu_{\beta}(\underline{A})$ for all $\beta \in \mathbb{B}$ and $A[p] / H$ is the canonical subgroup of $\underline{A} / H$.

(3) If there is a prime $\mathfrak{p} \mid p$, and $\beta, \beta^{\prime} \in \mathbb{B}_{\mathfrak{p}}$ such that we have $\nu_{\beta}(\underline{A})+p \nu_{\sigma^{-1} \circ \beta}(\underline{A}) \leq 1$ and $\nu_{\beta^{\prime}}(\underline{A})+p \nu_{\sigma^{-1} \circ \beta^{\prime}}(\underline{A}) \geq 1$, then $\underline{A} / H \notin \mathcal{U}$.

(4) Let $C$ be a subgroup of $\underline{A}$ which is anti-canonical at $\mathfrak{p}$. Then, $\nu_{\beta}(\underline{A} / C)=(1 / p) \nu_{\sigma \circ \beta}(\underline{A})$, for all $\beta \in \mathbb{B}_{\mathfrak{p}}$, and $A[p] / C$ is canonical at $\mathfrak{p}$. In particular, if $C$ is anti-canonical, then $\nu_{\beta}(\underline{A} / C)=(1 / p) \nu_{\sigma \circ \beta}(\underline{A})$, for all $\beta \in \mathbb{B}$ and $A[p] / C$ is the canonical subgroup of $\underline{A} / C$.

Proof. We let $Q=(\underline{A}, H)$ and $P=\pi(Q)=\underline{A}$.

(1): We have $Q \in \mathcal{V}_{\mathfrak{p}}$. For $\beta \in \mathbb{B}_{\mathfrak{p}}$, we write

$$
\nu_{\beta}(w Q)+p \nu_{\sigma^{-1} \circ \beta}(w Q)=1+p-\left(\nu_{\beta}(Q)+p \nu_{\sigma^{-1} \circ \beta}(Q)\right)=1+p-\left(\nu_{\beta}(P)+p \nu_{\sigma^{-1} \circ \beta}(P)\right)>p,
$$

using Proposition 4.2.2 and Lemma 5.3.4. This shows that $w Q \in \mathcal{W}_{\mathfrak{p}}$ and proves that $A[p] / H$ is anti-canonical at $\mathfrak{p}$. We can also write

$$
\nu_{\beta}(\underline{A} / H)=p\left(1-\nu_{\sigma^{-1} \circ \beta}(w Q)\right)=p \nu_{\sigma^{-1} \circ \beta}(Q)=p \nu_{\sigma^{-1} \circ \beta}(P)=p \nu_{\sigma^{-1} \circ \beta}(\underline{A}),
$$

using parts (2) and (1) of Lemma 5.3.4 for the first and third equalities, respectively.

(2): As above, we find $\nu_{\beta}(w Q)+p \nu_{\sigma^{-1} \circ \beta}(w Q)=1+p-\left(\nu_{\beta}(P)+p \nu_{\sigma^{-1} \circ \beta}(P)\right)<p$ for all $\beta \in \mathbb{B}_{\mathfrak{p}}$. Hence $w Q \in \mathcal{V}_{\mathfrak{p}}$, and $A[p] / H$ is canonical at $\mathfrak{p}$. For $\beta \in \mathbb{B}_{\mathfrak{p}}$, we have

$$
\nu_{\beta}(\underline{A} / H)=\nu_{\beta}(w Q)=1-\nu_{\beta}(Q)=1-\nu_{\beta}(P)=1-\nu_{\beta}(\underline{A}),
$$

using part (1) of Lemma 5.3.4, and the fact that both $Q$ and $w Q$ belong to $\mathcal{V}_{\mathfrak{p}}$.

(3): As above, we find that there is $\beta \in \mathbb{B}_{\mathfrak{p}}$ such that $\nu_{\beta}(w Q)+p \nu_{\sigma^{-1} \circ \beta}(w Q) \geq p$ and there is $\beta^{\prime} \in \mathbb{B}_{\mathfrak{p}}$ such that $\nu_{\beta^{\prime}}(w Q)+p \nu_{\sigma^{-1} \circ \beta^{\prime}}(w Q) \leq p$. This implies that $w Q \notin \mathcal{V}_{\mathfrak{p}} \cup \mathcal{W}_{\mathfrak{p}}$, and equivalently, $w Q \notin \mathcal{V} \cup \mathcal{W}$. By Corollary 5.3.7, we find that $\underline{A} / H=\pi(w Q) \notin \mathcal{U}$.

(4): Let $Q^{\prime}=(\underline{A}, C)$, and $P=\underline{A}=\pi\left(Q^{\prime}\right)$. By assumption, $Q^{\prime} \in \mathcal{W}_{\mathfrak{p}}$, and we can write

$$
\nu_{\beta}\left(w Q^{\prime}\right)+p \nu_{\sigma^{-1} \circ \beta}\left(w Q^{\prime}\right)=1+p-\left(\nu_{\beta}\left(Q^{\prime}\right)+p \nu_{\sigma^{-1} \circ \beta}\left(Q^{\prime}\right)\right)<1<p,
$$

for all $\beta \in \mathbb{B}_{\mathfrak{p}}$. Hence $w Q^{\prime} \in \mathcal{V}_{\mathfrak{p}}$ and $A[p] / C$ is canonical at $\mathfrak{p}$. For $\beta \in \mathbb{B}$ we write

$$
\nu_{\beta}(\underline{A} / C)=\nu_{\beta}\left(w Q^{\prime}\right)=1-\nu_{\beta}\left(Q^{\prime}\right)=(1 / p) \nu_{\sigma \circ \beta}(P)=(1 / p) \nu_{\sigma \circ \beta}(\underline{A}),
$$

using parts (1) and (2) of Lemma 5.3.4 for the first and third equalities, respectively.

Employing an iterative construction, we can use the above theorem to prove the existence of higher-order canonical subgroups.

Proposition 5.4.4. Let $\underline{A}$ defined over $K$ correspond to a point $P$ on $\mathfrak{X}_{\mathrm{rig}}$. Let $n$ be a nonnegative integer. Assume that

$$
\nu_{\beta}(\underline{A})+p \nu_{\sigma^{-1} \circ \beta}(\underline{A})<p^{1-n}, \quad \forall \beta \in \mathbb{B} .
$$


Then, for $1 \leq i \leq n+1$, there are isotropic finite flat subgroup schemes $H_{i}$ of $A$ of order $p^{i g}$, $\mathcal{O}_{L}$-invariant, and killed by $p^{i}$, forming an increasing sequence

$$
H_{1} \subset H_{2} \subset \cdots \subset H_{n+1}
$$

where $H_{1}$ is the canonical subgroup of $\underline{A}$, for any $1 \leq i \leq n$ we have $p^{i} H_{n+1}=H_{n+1-i}$, and each $H_{i}$ is a cyclic $\mathcal{O}_{L}$-module. Furthermore, if $P$ has non-cuspidal reduction, and if $r$ in $K$ is such that $\operatorname{val}(r)=\max \left\{\nu_{\beta}(\underline{A}): \beta \in \mathbb{B}\right\}$, then $H_{i}$ reduces modulo $p / r^{p^{i-1}}$ to $\operatorname{Ker}\left(\operatorname{Fr}^{i}\right)$.

Proof. The case $n=0$ is a consequence of the above results, and thus we can assume that $n \geq 1$. We construct this flag of subgroups recursively. For simplicity, we denote $\nu_{\beta}+p \nu_{\sigma^{-1} \circ \beta}$ by $\lambda_{\beta}$. Let $H_{0}=\{0\}$ and $H_{1}$ be the canonical subgroup of $\underline{A}$, which exists by Theorem 5.3.1. Fix $m<n+1$, and assume that for $1 \leq i \leq m$ there is a increasing sequence of subgroups

$$
H_{1} \subset \cdots \subset H_{i} \subset \cdots \subset H_{m},
$$

where for each $1 \leq i \leq m$, the subgroup $H_{i}$ has the stated properties, and, additionally:

(1) $\lambda_{\beta}\left(\underline{A} / H_{i}\right)<p^{1-n+i}$ for all $\beta \in \mathbb{B}$;

(2) $H_{i} / H_{i-1}$ is the canonical subgroup of $\underline{A} / H_{i-1}$;

(3) $p^{j} H_{i}=H_{i-j}$ for all $0 \leq j \leq i$.

This holds for $m=1$ by part (1) of Theorem $\left[5.4 .3\right.$, since $\lambda_{\beta}(\underline{A})<p^{1-n} \leq 1$, for all $\beta \in \mathbb{B}$.

Given the above data, we construct $H_{m+1}$. Since $\lambda_{\beta}\left(\underline{A} / H_{m}\right)<p^{1-n+m} \leq p, \underline{A} / H_{m}$ has a canonical subgroup of the form $H_{m+1} / H_{m}$, where $H_{m+1}$ is an isotropic finite flat subgroup scheme of $A$ of order $p^{(m+1) g}$ containing $H_{m}$ and killed by $p^{m+1}$. We show that $p H_{m+1}=H_{m}$. Since, by construction, $p H_{m+1} \subseteq H_{m}$, it is enough to show that $H_{m+1} \cap A[p]=H_{1}$. But since $\lambda_{\beta}\left(\underline{A} / H_{m-1}\right)<p^{-n+m} \leq 1$, we can apply part (1) of Theorem 5.4.3 to $A / H_{m-1}$ and its canonical subgroup $H_{m} / H_{m-1}$ to deduce that $\left(A[p]+H_{m}\right) / H_{m}$ and $H_{m+1} / H_{m}$ have trivial intersection, which proves the claim. To carry the induction forward, we only need to prove that when $m+1<n+1$, we have $\lambda_{\beta}\left(\underline{A} / H_{m+1}\right)<p^{1-n+m+1}$ for all $\beta \in \mathbb{B}$. That follows, since when $m+1<n+1$, we have $\lambda_{\beta}\left(\underline{A} / H_{m}\right)<p^{1-n+m} \leq 1$, and hence, by part (1) of Theorem 5.4 .3 , we have $\nu_{\beta}\left(\underline{A} / H_{m+1}\right)=p \nu_{\sigma^{-1} \circ \beta}\left(\underline{A} / H_{m}\right)$ for all $\beta \in \mathbb{B}$.

The final statement follows from the iterative construction and part (1) of Theorem 5.4.2.

\section{Functoriality}

There are two kinds of functoriality associated with the moduli spaces $X, Y$, and the many maps we have defined in that context. One kind of functoriality is coming from the moduli problem itself and is based on the construction $A \mapsto A \otimes_{\mathcal{O}_{L}} \mathcal{O}_{M}$ associating to an abelian variety $A$ with $\mathrm{RM}$ by $\mathcal{O}_{L}$ another abelian variety with $\mathrm{RM}$ by $\mathcal{O}_{M}$ for an extension of totally real fields $L \subseteq M$. We show that the canonical subgroup behaves naturally relative to this construction and deduce a certain optimality result for canonical subgroups (Corollary 6.1.1). The second kind of functoriality is relative to Galois automorhisms and comes from the fact that the moduli spaces $X, Y$, are in fact defined over $\mathbb{Z}_{p}$. There is thus a natural Galois action on our constructions that were made over $W(\kappa)$, which induces a descent data. This would allow us to show that the construction of section $s^{\dagger}$ descends to $\mathbb{Q}_{p}$. 
6.1. Changing the field. Let $L \subseteq M$ be totally real fields in which $p$ is unramified. Let $\mathbb{B}^{L}=\operatorname{Emb}\left(L, \overline{\mathbb{Q}}_{p}\right)$; similarly define $\mathbb{B}^{M}$. Given a subset $S \subseteq \mathbb{B}^{L}$, let $S^{M}=\left\{\beta \in \mathbb{B}^{M}: \beta_{\left.\right|_{L}} \in S\right\}$. In this section, we decorate the notation we have used so far with an $M$ or $L$ as a superscript. For example, we use $X^{L}, X^{M}, \pi^{L}$, etc. Given $Q=(\underline{A}, H) \in Y^{L}, P=\pi^{L}(Q) \in X^{L}$, we get points $\epsilon_{L, M}(Q)=\left(A \otimes_{\mathcal{O}_{L}} \mathcal{O}_{M}, H \otimes_{\mathcal{O}_{L}} \mathcal{O}_{M}\right) \in Y^{M}, \epsilon_{L, M}(P)=A \otimes_{\mathcal{O}_{L}} \mathcal{O}_{M} \in X^{M}$. This induces morphisms

$$
\epsilon=\epsilon_{L, M}: X^{L} \rightarrow X^{M}, \quad \epsilon=\epsilon_{L, M}: Y^{L} \rightarrow Y^{M},
$$

which extend to morphisms

$$
\epsilon=\epsilon_{L, M}: \mathbb{X}^{L} \rightarrow \mathbb{X}^{M}, \quad \epsilon=\epsilon_{L, M}: \mathbb{Y}^{L} \rightarrow \mathbb{Y}^{M}
$$

These morphisms fit into commutative diagrams:
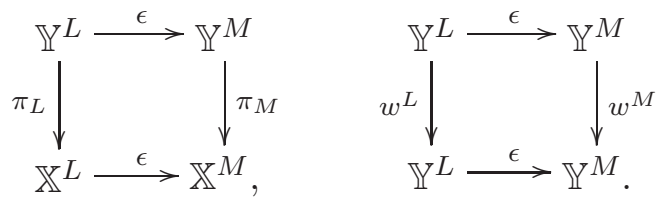

Let $k \supseteq \kappa$ be a perfect field. Let $\bar{P}, \bar{Q}$ be $k$-rational points of $\overline{\mathbb{X}}, \overline{\mathbb{Y}}$, respectively. Using that $\mathbb{D}(\epsilon(\underline{A})[p])=\mathbb{D}(\underline{A}[p]) \otimes_{\mathcal{O}_{L}} \mathcal{O}_{M}$, and so $\operatorname{Lie}(\epsilon(\underline{A}))=\operatorname{Lie}(\underline{A}) \otimes_{\mathcal{O}_{L}} \mathcal{O}_{M}$ etc., we find that

$$
\tau(\epsilon(\bar{P}))=\tau(\bar{P})^{M},
$$

and

$$
\varphi(\epsilon(\bar{Q}))=\varphi(\bar{Q})^{M}, \quad \eta(\epsilon(\bar{Q}))=\eta(\bar{Q})^{M}, \quad I(\epsilon(\bar{Q}))=I(\bar{Q})^{M} .
$$

Suppose that $\bar{Q} \in \bar{Y}(k)$, and $\bar{P} \in \bar{X}(k)$. It is clear from the discussion in 2.4 that one can choose parameters for $\widehat{\mathcal{O}}_{X^{L}, \bar{P}}, \widehat{\mathcal{O}}_{Y^{L}, \bar{Q}}, \widehat{\mathcal{O}}_{X^{M}, \epsilon(\bar{P})}, \widehat{\mathcal{O}}_{Y^{M}, \epsilon(\bar{Q})}$ as in loc. cit. so that, in addition, the map $\epsilon^{*}: \widehat{\mathcal{O}}_{X^{M}, \epsilon(\bar{P})} \rightarrow \widehat{\mathcal{O}}_{X^{L}, \bar{P}}$ satisfies

$$
\epsilon^{*}\left(t_{\beta}\right)=t_{\left(\left.\beta\right|_{L}\right)},
$$

and that $\epsilon^{*}: \widehat{\mathcal{O}}_{Y^{M}, \epsilon(\bar{Q})} \rightarrow \widehat{\mathcal{O}}_{Y^{L}, \bar{Q}}$ satisfies

$$
\epsilon^{*}\left(x_{\beta}\right)=x_{\left(\left.\beta\right|_{L}\right)}, \quad \epsilon^{*}\left(y_{\beta}\right)=y_{\left(\left.\beta\right|_{L}\right)}, \quad \epsilon^{*}\left(z_{\beta}\right)=z_{\left(\left.\beta\right|_{L}\right)} .
$$

It is then clear that the function $\Delta_{L, M}: \Theta^{L} \rightarrow \Theta^{M}$ given by $\left(a_{\beta}\right)_{\beta \in \mathbb{B}^{L}} \mapsto\left(b_{\beta}\right)_{\beta \in \mathbb{B}^{M}}$, where $b_{\beta}=$ $a_{\left(\left.\beta\right|_{L}\right)}$, fits into commutative diagrams:

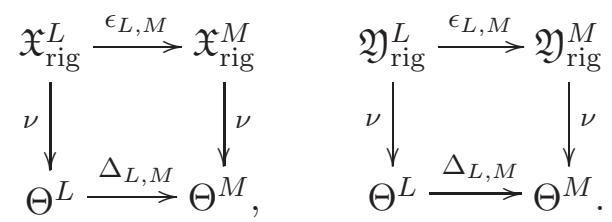

It follows immediately from the definitions and the discussion above that

(1) $\epsilon_{L, M}^{-1}\left(\mathcal{U}^{M}\right)=\mathcal{U}^{L}$, and similarly for $\mathcal{V}^{L}, \mathcal{V}^{M}$, and $\mathcal{W}^{L}, \mathcal{W}^{M}$.

(2) $\epsilon_{L, M}^{-1}\left(\mathcal{V}_{\mathfrak{p}}^{M}\right)=\mathcal{V}_{\mathfrak{p} \cap L}^{L}$. 
Corollary 6.1.1. Let $\mathcal{U}^{+} \supseteq \mathcal{U}^{L}$ be an admissible open of $\mathfrak{X}_{\text {rig }}^{L}$ containing $\nu_{\mathfrak{Y})^{L}}^{-1}\left(\frac{p}{p+1}, \frac{p}{p+1}, \ldots, \frac{p}{p+1}\right)$. The section $s^{\dagger, L}: \mathcal{U}^{L} \rightarrow \mathfrak{Y}_{\text {rig }}^{L}$ cannot be extended to $\mathcal{U}^{+}$.

Proof. If $s^{\dagger, L}$ can be extended to $\mathcal{U}^{+}$, then the above functoriality results would imply that $s^{\dagger, \mathbb{Q}}: \mathcal{U}^{\mathbb{Q}} \rightarrow \mathfrak{Y}_{\text {rig }}^{\mathbb{Q}}$ can be extended to $\epsilon_{\mathbb{Q}, L}^{-1}\left(\mathcal{U}^{+}\right) \supseteq \mathcal{U}^{\mathbb{Q}}$, which contains $\nu_{\mathfrak{Y}^{\mathbb{Q}}}^{-1}\left(\frac{p}{p+1}\right)$. This is impossible by [20, Theorem 3.9].

6.2. Galois automorphisms. We next discuss the action of $\operatorname{Gal}\left(\overline{\mathbb{Q}}_{p} / \mathbb{Q}_{p}\right)$ on $\mathbb{X}, \mathbb{Y}$, and all the derived maps. The action is a result of the identifications $\mathbb{X}=\mathbb{X}_{\mathbb{Z}_{p}} \otimes_{\mathbb{Z}_{p}} W(\kappa), \mathbb{Y}=\mathbb{Y}_{\mathbb{Z}_{p}} \otimes_{\mathbb{Z}_{p}} W(\kappa)$, while our constructions used the $W(\kappa)$-structure of $\mathbb{X}, \mathbb{Y}$. The following facts are easy to verify.

(1) $\operatorname{Gal}\left(\overline{\mathbb{Q}}_{p} / \mathbb{Q}_{p}\right)$ acts on $\mathbb{B}$ by composition and acts transitively on each $\mathbb{B}_{\mathfrak{p}}$. Let $\gamma \in \operatorname{Gal}\left(\overline{\mathbb{Q}}_{p} / \mathbb{Q}_{p}\right)$; it induces the following maps:

- $\gamma: W(\kappa) \rightarrow W(\kappa)$,

- $\gamma^{*}: \operatorname{Spec}(W(\kappa)) \rightarrow \operatorname{Spec}(W(\kappa))$,

- $1 \times \gamma^{*}: \mathbb{Y}=\mathbb{Y}_{\mathbb{Z}_{p}} \times_{\operatorname{Spec}\left(\mathbb{Z}_{p}\right)} \operatorname{Spec}(W(\kappa)) \rightarrow \mathbb{Y}=\mathbb{Y}_{\mathbb{Z}_{p}} \times_{\operatorname{Spec}\left(\mathbb{Z}_{p}\right)} \operatorname{Spec}(W(\kappa))$.

- $1 \times \gamma^{*}: \mathbb{X}=\mathbb{X}_{\mathbb{Z}_{p}} \times_{\operatorname{Spec}\left(\mathbb{Z}_{p}\right)} \operatorname{Spec}(W(\kappa)) \rightarrow \mathbb{X}=\mathbb{X}_{\mathbb{Z}_{p}} \times_{\operatorname{Spec}\left(\mathbb{Z}_{p}\right)} \operatorname{Spec}(W(\kappa))$.

Moreover, the following diagram is commutative:

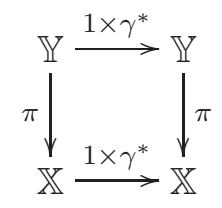

(and similarly for $\mathfrak{X}_{\text {rig }}, \mathfrak{Y}_{\text {rig }}$ ).

(2) For $\bar{Q}$ a closed point of $\overline{\mathbb{Y}}$, and $\bar{P}$ a closed point of $\overline{\mathbb{X}}$ :

- $\varphi\left(1 \times \gamma^{*}(\bar{Q})\right)=\gamma(\varphi(\bar{Q}))$,

- $\eta\left(1 \times \gamma^{*}(\bar{Q})\right)=\gamma(\eta(\bar{Q}))$,

- $I\left(1 \times \gamma^{*}(\bar{Q})\right)=\gamma(I(\bar{Q}))$,

- $\tau\left(1 \times \gamma^{*}(\bar{P})\right)=\gamma(\tau(\bar{P}))$,

where for $S \subseteq \mathbb{B}, \gamma(S)=\{\gamma \circ \beta: \beta \in S\}$.

(3) Let $\gamma: \Theta \rightarrow \Theta$ be given by $\left(a_{\beta}\right)_{\beta \in \mathbb{B}} \mapsto\left(b_{\beta}\right)_{\beta \in \mathbb{B}}$, where $b_{\beta}=a_{\gamma^{-1} \beta}$. Then, the following diagram is commutative:

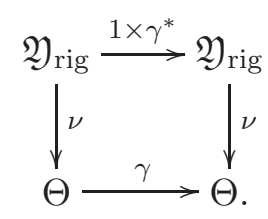

The same statement holds for $\mathfrak{X}_{\text {rig. }}$.

(4) It follows immediately from the definitions and the statements above that

- $1 \times \gamma^{*}(\mathcal{U})=\mathcal{U}$,

- $1 \times \gamma^{*}(\mathcal{V})=\mathcal{V}$

- $1 \times \gamma^{*}(\mathcal{W})=\mathcal{W}$. 
To be able to use our results on the geometry of $\overline{\mathbb{Y}}, \overline{\mathbb{X}}$, and $\pi: \overline{\mathbb{Y}} \rightarrow \overline{\mathbb{X}}$, we found it convenient to work over $W(\kappa)$ throughout the paper. The canonical section $s^{\dagger}$, however, can be shown to exist over $\mathbb{Q}_{p}$.

Theorem 6.2.1. There are admissible opens $\mathcal{U}_{\mathbb{Q}_{p}} \subset \mathfrak{X}_{\mathrm{rig}, \mathbb{Q}_{p}}$ and $\mathcal{V}_{\mathbb{Q}_{p}} \subset \mathfrak{Y}_{\text {rig, } \mathbb{Q}_{p}}$ whose base changes to $\mathbb{Q}_{\kappa}$ are $\mathcal{U}, \mathcal{V}$, respectively, and a section

$$
s_{\mathbb{Q}_{p}}^{\dagger}: \mathcal{U}_{\mathbb{Q}_{p}} \rightarrow \mathfrak{Y}_{\text {rig, }, \mathbb{Q}_{p}}
$$

to $\pi_{\mathbb{Q}_{p}}: \mathfrak{Y}_{\text {rig, } \mathbb{Q}_{P}} \rightarrow \mathfrak{X}_{\text {rig, } \mathbb{Q}_{p}}$ whose image is $\mathcal{V}_{\mathbb{Q}_{p}}$, and whose base change to $\mathbb{Q}_{\kappa}$ is $s^{\dagger}$.

Proof. We may think about the points of $\mathfrak{Y}_{\text {rig }}$, or $\mathfrak{X}_{\text {rig }}$, as Galois orbits of the points of $\mathfrak{Y}$ rig, or $\mathfrak{X}_{\text {rig }}$, over $\overline{\mathbb{Q}}_{p}$, relative to the action of $\operatorname{Gal}\left(\overline{\mathbb{Q}}_{p} / \mathbb{Q}_{p}\right)$, and so the points of $\mathfrak{Y}$ rig, $\mathbb{Q}_{p}$ are the Galois orbits of the points of $\mathfrak{Y}_{\text {rig }}$ relative to $\operatorname{Gal}\left(\mathbb{Q}_{\kappa} / \mathbb{Q}_{p}\right)$. It is then clear that the section $s^{\dagger}$ is welldefined on such orbits (cf. Diagram 6.2.1). As a set, $\mathcal{U}_{\mathbb{Q}_{p}}$ comprises the $\operatorname{Gal}\left(\mathbb{Q}_{\kappa} / \mathbb{Q}_{p}\right)$-orbits of the points of $\mathcal{U}$; similarly, for $\mathcal{V}_{\mathbb{Q}_{p}}$.

Two points may be worth mentioning. The admissibility of $\mathcal{U}_{\mathbb{Q}_{p}}$ boils down to the fact $\mathcal{U}$ is a union of affinoids invariant under the Galois action (since they are defined by valuation conditions), and that an ideal of a $\mathbb{Q}_{\kappa}$-Tate algebra which is invariant under $\operatorname{Gal}\left(\mathbb{Q}_{\kappa} / \mathbb{Q}_{p}\right)$ is

generated by power series with coefficients in $\mathbb{Q}_{p}$. The fact that $s_{\mathbb{Q}_{p}}^{\dagger}$ is a morphism boils down to the fact that for two $\mathbb{Q}_{p}$-affinoids $\mathcal{A}, \mathcal{B}$ we have $\operatorname{Hom}(\mathcal{A}, \mathcal{B})=\operatorname{Hom}_{K}(\mathcal{A} \otimes K, \mathcal{B} \otimes K)^{\operatorname{Gal}\left(K / \mathbb{Q}_{p}\right)}$ for any finite Galois extension $K / \mathbb{Q}_{p}$. Both these statements are easy to check.

\section{ApPENDIX}

In this section, we construct variants of the moduli space $Y$ considered in the paper so far. These moduli spaces are natural and useful to the construction of partial $U$ operators $\left\{U_{\mathfrak{p}}\right\}$. Since the results are very similar to the results obtained henceforth, we shall be very brief, except for very specific results not mentioned previously.

7.1. A variant on the moduli problem. Let $\mathfrak{t}$ be an ideal of $\mathcal{O}_{L}$ dividing $p, \mathfrak{t}^{*}=p / \mathfrak{t}$ (so $\left.\mathfrak{t t}^{*}=p \mathcal{O}_{L}\right), \mathbb{B}_{\mathfrak{t}}=\cup_{\mathfrak{p} \mid \mathfrak{t}} \mathbb{B}_{\mathfrak{p}}$, and $f(\mathfrak{t})=\sum_{\mathfrak{p} \mid \mathfrak{t}} f(\mathfrak{p})$ the sum of the residue degrees. Consider the functor associating to a $W(\kappa)$-scheme, the isomorphism classes of $(\underline{A}, H)$, where $\underline{A}$ is an object parameterized by $X$, and where $H \subseteq A[p]$ is an isotropic $\mathcal{O}_{L}$-invariant finite-flat subgroup scheme of rank $p^{f(\mathfrak{t})}$, on which $\mathfrak{t}$ acts as zero; $H$ has a well-defined action of $\mathcal{O}_{L} / \mathfrak{t} \cong \oplus_{\mathfrak{p} \mid \mathfrak{t}} \mathcal{O}_{L} / \mathfrak{p}$. There is a fine moduli scheme $Y(\mathfrak{t})$ representing this functor, which actually has a model over $\mathbb{Z}_{\mathfrak{p}}$; it affords a minimal compactification denoted by $\mathbb{Y}(\mathfrak{t})$. In what follows, $\bar{Y}(\mathfrak{t}), \bar{Y}(\mathfrak{t}), \mathfrak{Y}$ rig $(\mathfrak{t})$ are defined similar to the case $\mathfrak{t}=p \mathcal{O}_{L}$ studied throughout the paper.

The natural morphism

$$
\pi(\mathfrak{t}): \mathbb{Y}(\mathfrak{t}) \rightarrow \mathbb{X}
$$


is proper, and finite-flat over $\mathbb{Q}_{\kappa}$ of degree $\prod_{\mathfrak{p} \mid \mathfrak{t}}\left(p^{f(\mathfrak{p})}+1\right)$. Given $\underline{A}$, there is a canonical decomposition $A[p]=\prod_{\mathfrak{p} \mid p} A[\mathfrak{p}]$, where $A[\mathfrak{p}]=\cap_{a \in \mathfrak{p}} \operatorname{Ker}(\iota(a))$ is a finite flat group scheme of rank $p^{f(\mathfrak{p})}$. The subgroup $H$ appearing above is a subgroup of $A[\mathfrak{t}]:=\prod_{\mathfrak{p} \mid \mathfrak{t}} A[\mathfrak{p}]$. Suppose $A$ is over a characteristic $p$ base; let $\operatorname{Ker}(\operatorname{Fr})[\mathfrak{t}]=\operatorname{Ker}(\mathrm{Fr}) \cap A[\mathfrak{t}]$. It is an example of a subgroup scheme of the kind parameterized by $Y(\mathfrak{t})$ (cf. Lemma 2.1.1). There is a natural section

$$
s(\mathfrak{t}): \overline{\mathbb{X}} \rightarrow \overline{\mathbb{Y}}(\mathfrak{t})
$$

given on non-cuspidal points by

$$
\underline{A} \mapsto(\underline{A}, \operatorname{Ker}(\operatorname{Fr})[\mathfrak{t}]) .
$$

We can also present the moduli problem in a "balanced" way. It requires making auxiliary choices. The ideal $\mathfrak{t}$ has a natural notion of positivity, and so it acts on the representatives $\left[\mathrm{Cl}^{+}(L)\right]$ for the strict class group of $L$. Choose for every such representative $\left(\mathfrak{a}, \mathfrak{a}^{+}\right)$an isomorphism $\gamma_{\mathfrak{t},\left(\mathfrak{a}, \mathfrak{a}^{+}\right)}$of $\mathfrak{t} \cdot\left(\mathfrak{a}, \mathfrak{a}^{+}\right)$with another (uniquely determined) representative in $\left[\mathrm{Cl}^{+}(L)\right]$. (In the case considered in the body of the paper $\mathfrak{t}=(p)$, and there is a canonical isomorphism $p \cdot\left(\mathfrak{a}, \mathfrak{a}^{+}\right) \cong\left(\mathfrak{a}, \mathfrak{a}^{+}\right)$, which is dividing by $p$.)

Consider the moduli of $(f: \underline{A} \rightarrow \underline{B})$ such that $H=\operatorname{Ker}(f)$ is an $\mathcal{O}_{L}$-invariant isotropic finiteflat subgroup scheme of $A[\mathfrak{t}]$ of order $p^{f(\mathfrak{t})}$, such that $f^{*} \mathcal{P}_{\underline{B}}=\mathfrak{t} \mathcal{P}_{\underline{A}}$, and $\lambda_{B}=\gamma_{\mathfrak{t},\left(\mathfrak{a}, \mathfrak{a}^{+}\right)} \circ \lambda_{A}$. There is an automorphism

$$
w: \mathbb{Y}(\mathfrak{t}) \rightarrow \mathbb{Y}(\mathfrak{t}),
$$

constructed as follows. Given $(\underline{A}, H)$ consider $\underline{A} / H$ and its subgroup scheme $A[p] / H$; take its $\mathfrak{t}$-primary part $(A[p] / H)[\mathfrak{t}]$. We let

$$
w(\underline{A}, H)=(\underline{A} / H,(A[p] / H)[\mathfrak{t}]) .
$$

In terms of the presentation $(f: \underline{A} \rightarrow \underline{B})$, we first find a unique isogeny $f^{t}: \underline{B} \rightarrow \underline{A}$, such that

$$
f^{t} \circ f=[p] \text {. }
$$

We then replace $\operatorname{Ker}\left(f^{t}\right)$ by its $\mathfrak{t}$-primary part to obtain the subgroup scheme $(A[p] / H)[\mathfrak{t}]$. We note that $w$ permutes the connected components of $\mathbb{Y}(\mathfrak{t})$; it changes the polarization module.

Given $(\underline{A}, H)$, we let $B=\underline{A} / H, f: \underline{A} \rightarrow \underline{B}$ the canonical map, and consider the diagrams:

$$
\begin{aligned}
& \bigoplus_{\beta \in \mathbb{B}} \operatorname{Lie}(f)_{\beta}: \bigoplus_{\beta \in \mathbb{B}} \operatorname{Lie}(\underline{A})_{\beta} \longrightarrow \bigoplus_{\beta \in \mathbb{B}} \operatorname{Lie}(\underline{B})_{\beta}, \\
& \bigoplus_{\beta \in \mathbb{B}} \operatorname{Lie}\left(f^{t}\right)_{\beta}: \bigoplus_{\beta \in \mathbb{B}} \operatorname{Lie}(\underline{B})_{\beta} \longrightarrow \bigoplus_{\beta \in \mathbb{B}} \operatorname{Lie}(\underline{A})_{\beta} .
\end{aligned}
$$

We let, as before,

$$
\begin{aligned}
& \varphi(\underline{A}, H)=\left\{\beta \in \mathbb{B}_{\mathfrak{t}}: \operatorname{Lie}(f)_{\sigma^{-1} \circ \beta}=0\right\}, \\
& \eta(\underline{A}, H)=\left\{\beta \in \mathbb{B}_{\mathfrak{t}}: \operatorname{Lie}\left(f^{t}\right)_{\beta}=0\right\}, \\
& I(\underline{A}, H)=\ell(\varphi(f)) \cap \eta(f)=\left\{\beta \in \mathbb{B}: \operatorname{Lie}(f)_{\beta}=\operatorname{Lie}\left(f^{t}\right)_{\beta}=0\right\} .
\end{aligned}
$$

Note that $\varphi(\underline{A}, H)$ in fact equals $\left\{\beta \in \mathbb{B}: \operatorname{Lie}(f)_{\sigma^{-1} \circ \beta}=0\right\}$, while $\left\{\beta \in \mathbb{B}: \operatorname{Lie}\left(f^{t}\right)_{\beta}=0\right\}$ always contains $\mathbb{B}_{t^{*}}$, which is "superfluous information". For that reason, and for having nicer formulas in what follows, we have defined $\eta(\underline{A}, H)$ using only $\mathbb{B}_{\mathrm{t}}$. 
One checks that these invariants do not depend on the choice of $\gamma_{\mathfrak{t},\left(\mathfrak{a}, \mathfrak{a}^{+}\right)}$. A pair $(\varphi, \eta)$ of subsets of $\mathbb{B}_{\mathfrak{t}}$ is called $\mathfrak{t}$-admissible if $\mathbb{B}_{\mathfrak{t}}-\ell(\varphi) \subseteq \eta$. There are $3^{f(\mathfrak{t})}$ such pairs.

We define, as before, subsets $W_{\varphi, \eta}(\mathfrak{t}), Z_{\varphi, \eta}(\mathfrak{t})$ of $\bar{Y}(\mathfrak{t})$ for $\mathfrak{t}$-admissible pairs $(\varphi, \eta)$, and their natural extensions $\mathbb{W}_{\varphi, \eta}(\mathfrak{t}), \mathbb{Z}_{\varphi, \eta}(\mathfrak{t})$ to $\overline{\mathbb{Y}}(\mathfrak{t})$. This gives us, as before, a stratification of $\overline{\mathbb{Y}}(\mathfrak{t})$, with very similar properties to the stratification studied in the body of the paper; the reader should have no difficulty listing its properties 1

The various moduli spaces $\mathbb{Y}(\mathfrak{t})$ satisfy the following compatibility relation: for $\mathfrak{t}, \mathfrak{z}$ relatively prime ideals of $\mathcal{O}_{L}$ dividing $p$ we have

$$
\mathbb{Y}(\mathfrak{t} \mathfrak{z})=\mathbb{Y}(\mathfrak{t}) \times \mathbb{X} \mathbb{Y}(\mathfrak{z}) .
$$

Let $(\varphi, \eta)$ be a $\mathfrak{t}$-admissible pair and $\left(\varphi^{\prime}, \eta^{\prime}\right)$ a $\mathfrak{z}$-admissible pair. Then

$$
\mathbb{W}_{\varphi, \eta}(\mathfrak{t}) \times_{\mathbb{X}} \mathbb{W}_{\varphi^{\prime}, \eta^{\prime}}(\mathfrak{z})=\mathbb{W}_{\varphi \cup \varphi^{\prime}, \eta \cup \eta^{\prime}}\left(\mathfrak{t}_{\mathfrak{z}}\right), \quad \mathbb{Z}_{\varphi, \eta}(\mathfrak{t}) \times_{\mathbb{X}} \mathbb{Z}_{\varphi^{\prime}, \eta^{\prime}}(\mathfrak{z})=\mathbb{Z}_{\varphi \cup \varphi^{\prime}, \eta \cup \eta^{\prime}}\left(\mathfrak{t}_{\mathfrak{z}}\right) .
$$

Given two points $(\underline{A}, H),\left(\underline{A}, H^{\prime}\right)$ on $\bar{Y}(\mathfrak{t})$ and $\bar{Y}(\mathfrak{z})$, respectively, our invariants involve two different quotients of $\underline{A}$. One verifies (17.1.6) by considering the following cartesian diagram:

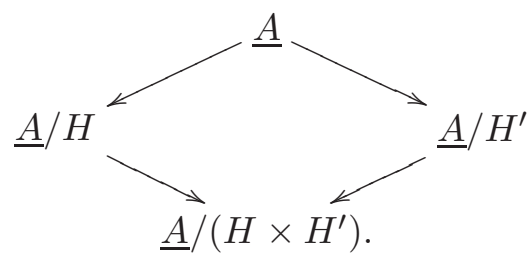

Alternately, one can prove a lemma similar to Lemma 2.3.3, which describes the invariants in terms of the relative position of $\operatorname{Fr}(\mathbb{D}(A[p])), \operatorname{Ver}(\mathbb{D}(A[p]))$, and the submodule $M$ of $\mathbb{D}(A[p])$ such that $\mathbb{D}(A[p]) / M=\mathbb{D}(H)$.

Define:

$$
\overline{\mathbb{Y}}(\mathfrak{p})_{F}:=\mathbb{Z}_{\mathbb{B}_{\mathfrak{p}}, \emptyset}(\mathfrak{p}), \quad \overline{\mathbb{Y}}(\mathfrak{p})_{V}:=\mathbb{Z}_{\emptyset, \mathbb{B}_{\mathfrak{p}}}(\mathfrak{p}) .
$$

In fact, we have $\overline{\mathbb{Y}}(\mathfrak{p})_{F}=s(\mathfrak{p})(\overline{\mathbb{X}})$, and $\overline{\mathbb{Y}}(\mathfrak{p})_{V}=w\left(\overline{\mathbb{Y}}(\mathfrak{p})_{F}\right)$. For every ideal $\mathfrak{t} \mid p$, we have

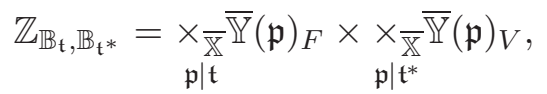

by Equation (7.1.6). In particular, the choice of $\mathfrak{t}=p$ gives $\overline{\mathbb{Y}}_{F}$, and the choice of $\mathfrak{t}=\mathcal{O}_{L}$ gives $\overline{\mathbb{Y}}_{V}$.

Lemma 7.1.1. The morphism

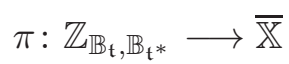

is finite, flat, and purely inseparable of degree $p^{f\left(\mathfrak{t}^{*}\right)}$.

Proof. To prove the lemma, it is enough to prove that the morphisms

$$
\pi(\mathfrak{p}): \overline{\mathbb{Y}}(\mathfrak{p})_{F} \longrightarrow \overline{\mathbb{X}}, \quad \pi(\mathfrak{p}): \overline{\mathbb{Y}}(\mathfrak{p})_{V} \longrightarrow \overline{\mathbb{X}}
$$

\footnotetext{
${ }^{1}$ It should be remarked that since the strata can be defined by vanishing of sections of line bundles, they have a natural scheme structure and are, in fact, reduced. Interestingly, that way one can see that each strata of $\overline{\mathbb{Y}}(\mathfrak{t})$ (and also of $\overline{\mathbb{X}}$ ) represents a moduli problem, where the additional data is precisely the vanishing of the sections referred to above.
} 
obtained by restriction from $\overline{\mathbb{Y}}(\mathfrak{p})$, are finite-flat of degree 1 and $p^{f(\mathfrak{p})}$, respectively. We first consider the situation without compactifications; we have the morphisms

$$
\pi(\mathfrak{p}): \bar{Y}(\mathfrak{p})_{F} \longrightarrow \bar{X}, \quad \pi(\mathfrak{p}): \bar{Y}(\mathfrak{p})_{V} \longrightarrow \bar{X}
$$

Since the morphism $\pi(\mathfrak{p}): \bar{Y}(\mathfrak{p}) \rightarrow \bar{X}$ is proper and quasi-finite on each of $\bar{Y}(\mathfrak{p})_{F}, \bar{Y}(\mathfrak{p})_{V}$, it is in fact finite [22, Chapter $3, \S 4$, Proposition 4.4.2]. Since a finite surjective morphism of nonsingular varieties over an algebraically closed field is flat [24, Chapter III, Exercise 9.3(a)]. It follows that indeed we have two finite flat morphisms. The existence of a section $\bar{X} \rightarrow \bar{Y}(\mathfrak{p})_{F}$ shows the morphism $\bar{Y}(\mathfrak{p})_{F} \rightarrow \bar{X}$ is an isomorphism. The generic fibre of $Y(\mathfrak{p}) \rightarrow X$ is finite flat of degree $p^{f(\mathfrak{p})}+1$, and so it follows that the morphism $\bar{Y}(\mathfrak{p})_{V} \rightarrow \bar{X}$ is finite flat of degree $p^{f(\mathfrak{p})}$. Since the reduced fibers of this morphism are just singletons, we conclude that $\bar{Y}(\mathfrak{p})_{V} \rightarrow \bar{X}$ is purely inseparable of degree $p^{f(\mathfrak{p})}$.

We now indicate how to extend the argument to compactifications. One can use the description of the completed local ring of a cusp via $q$-expansions. Let $c^{\prime}$ be a cusp of $\overline{\mathbb{Y}}, c=\pi\left(c^{\prime}\right)$, viewed over $\overline{\mathbb{F}}_{p}$, lying on the connected component of $\bar{Y}$, respectively, $\overline{\mathbb{X}}$, corresponding to a polarization module $\left(\mathfrak{a}, \mathfrak{a}^{+}\right)$. To $c$ one associates a pair $(T, \Lambda)$, of translation module and multiplier group, determined by the group $\Gamma \subset \mathrm{SL}_{2}(L)$ corresponding to the given polarization datum and level $\Gamma_{00}(N)$ (see [17, I.£2-3]). Taking instead the subgroup $\Gamma^{\prime}$ of $\Gamma$, corresponding to adding the level structure at $p$, one obtains another pair $\left(T^{\prime}, \Lambda^{\prime}\right)$, where $T^{\prime}, \Lambda^{\prime}$ are finite index subgroups of $T, \Lambda$, respectively. The completed local ring of $c$ on $\overline{\mathbb{X}}$ is $\overline{\mathbb{F}}_{p} \llbracket q^{\nu} \rrbracket_{\nu \in T^{\vee}}^{\Lambda}$ and on $\overline{\mathbb{Y}}$ is $\overline{\mathbb{F}}_{p} \llbracket q^{\nu} \rrbracket_{\nu \in T^{\prime}}^{\Lambda^{\prime}}$ (see loc. cit. and [8]). Checking flatness at the cusp $c$ amounts to checking that $\overline{\mathbb{F}}_{p} \llbracket q^{\nu} \rrbracket_{\nu \in T^{\prime}}^{\Lambda^{\prime}}$ is a free $\overline{\mathbb{F}}_{p} \llbracket q^{\nu} \rrbracket_{\nu \in T^{\vee}}^{\Lambda}$-module, which can be verified by a straightforward calculation.

Lemma 7.1.2. Let $\bar{Q} \in W_{\mathbb{B}_{\mathfrak{t}}, \mathbb{B}_{\mathfrak{t}^{*}}}$, and $\bar{P}=\pi(\bar{Q})$. We can choose isomorphisms as in (2.8.2) at $\bar{P}$, and 2.8.4 at $\bar{Q}$, such that:

$$
\pi^{*}\left(t_{\beta}\right)= \begin{cases}z_{\beta} & \beta \in \mathbb{B}_{\mathfrak{t}} \\ z_{\beta}^{p} & \beta \in \mathbb{B}_{\mathfrak{t}^{*}} .\end{cases}
$$

Proof. First, we prove the lemma for the cases $\mathfrak{t}=p \mathcal{O}_{L}$ (i.e., $\bar{Q} \in \bar{Y}_{F}^{\text {ord }}$ ), and $\mathfrak{t}=\mathcal{O}_{L}$ (i.e., $\bar{Q} \in \bar{Y}_{V}^{\text {ord }}$ ). In the first case, the result follows from Lemma 2.7.3 and the fact that $\pi^{*}$ is an inverse to $s^{*}$.

Assume now that $\bar{Q} \in \bar{Y}_{V}^{\text {ord }}$. Consider the morphism $\pi \circ w \circ s: \bar{X}_{\mathbb{F}_{p}} \rightarrow \bar{X}_{\mathbb{F}_{p}}$. Calculating the effect of this map on points, we find that it is equal to the Frobenius morphism Fr: $\bar{X}_{\mathbb{F}_{p}} \rightarrow \bar{X}_{\mathbb{F}_{p}}$. Let $\bar{Q}_{1}$ be a point in $\bar{Y}_{F}^{\text {ord }}$ such that $w\left(\bar{Q}_{1}\right)=\bar{Q}$ and let $\bar{P}_{1}=\pi\left(\bar{Q}_{1}\right)$; then $I\left(\bar{Q}_{1}\right)=\emptyset$, and $\bar{P}=\operatorname{Fr}\left(\bar{P}_{1}\right)$. By Lemma 2.7.3. there are choices of isomorphisms as in (2.8.2) at $\bar{P}_{1}$, and (2.8.4) at $\bar{Q}_{1}$, such that $s^{*}\left(z_{\beta, \bar{Q}_{1}}\right)=t_{\beta, \bar{P}_{1}}$. We may choose parameters $\left\{t_{\beta, \bar{P}}\right\}_{\beta}$ at $\bar{P}$, such that $\operatorname{Fr}^{*}\left(t_{\beta, \bar{P}}\right)=t_{\beta, \bar{P}_{1}}^{p}$, and by Lemma 2.7.2. we can find parameters as in (2.8.4) at $\bar{Q}$, such that $w^{*}\left(z_{\beta, \bar{Q}}\right)=z_{\beta, \bar{Q}_{1}}$. Therefore,

$$
s^{*} \circ w^{*}\left(z_{\beta, \bar{Q}}^{p}\right)=t_{\beta, \bar{P}_{1}}^{p} .
$$

On the other hand, from the above discussion, we have

$$
s^{*} \circ w^{*} \circ \pi^{*}\left(t_{\beta, \bar{P}}\right)=\operatorname{Fr}^{*}\left(t_{\beta, \bar{P}}\right)=t_{\beta, \bar{P}_{1}}^{p} .
$$


Since $s^{*} \circ w^{*}$ is an isomorphism, it follows that $\pi^{*}\left(t_{\beta, \bar{P}}\right)=z_{\beta, \bar{Q}}^{p}$.

For a general horizontal stratum, we argue as follows. The arguments in 2.4 can be repeated ad verbatim for $\bar{Y}(\mathfrak{p})$ to produce parameters $\left\{x_{\beta, \mathfrak{p}}: \beta \in \mathbb{B}_{\mathfrak{p}} ; y_{\beta, \mathfrak{p}}: \beta \in \mathbb{B}_{\mathfrak{p}^{*}}\right\}$ at a point in $W_{\mathbb{B}_{\mathfrak{p}}, \mathbb{B}_{\mathfrak{p}}^{*}}(\mathfrak{p})$ (in the notation of the first formulation of Theorem 2.4.1). In fact, these parameters can be chosen compatibly with the parameters on $\bar{Y}$; more precisely, we have the following. Let $\bar{Q}$ be a closed point in $W_{\mathbb{B}_{\mathfrak{t}}, \mathbb{B}_{\mathfrak{t}^{*}}}$; let $\bar{Q}_{\mathfrak{p}}$ be the image in $\bar{Y}(\mathfrak{p})$ and $\bar{P}=\pi(\bar{Q})=\pi(\mathfrak{p})\left(\bar{Q}_{\mathfrak{p}}\right)$. We have an isomorphism:

$$
\widehat{\mathcal{O}}_{\bar{Y}, \bar{Q}} \cong \otimes_{\widehat{\mathcal{O}}_{\bar{X}, \bar{P}}} \widehat{\mathcal{O}}_{\bar{Y}(\mathfrak{p}), \bar{Q}_{\mathfrak{p}}}
$$

where the tensor product is over all prime ideals $\mathfrak{p}$ dividing $p$. In terms of the above parameters, this isomorphism can be written as:

$$
k \llbracket x_{\beta}: \beta \in \mathbb{B}_{\mathfrak{t}} ; y_{\beta}: \beta \in \mathbb{B}_{\mathfrak{t}^{*}} \rrbracket \cong \otimes_{\mathfrak{p} \mid \mathfrak{t}} k \llbracket x_{\beta, \mathfrak{p}}: \beta \in \mathbb{B}_{\mathfrak{p}} ; y_{\beta, \mathfrak{p}}: \beta \in \mathbb{B}_{\mathfrak{p}^{*}} \rrbracket \otimes \otimes_{\mathfrak{p} \mid \mathfrak{t}^{*}} k \llbracket y_{\beta, \mathfrak{p}}: \beta \in \mathbb{B} \rrbracket,
$$

where the tensor products are over $\widehat{\mathcal{O}}_{\bar{X}, \bar{P}} \cong k \llbracket t_{\beta} ; \beta \in \mathbb{B} \rrbracket$, and the images of $x_{\beta}, y_{\beta}$ are given, respectively, by $x_{\beta, \mathfrak{p}}, y_{\beta, \mathfrak{p}}$, where $\mathfrak{p}$ is the unique prime ideal such that $\beta \in \mathbb{B}_{\mathfrak{p}}$. This allows us to partially calculate the morphism

$$
\pi(\mathfrak{p})^{*}: \widehat{\mathcal{O}}_{\bar{X}, \bar{P}} \rightarrow \widehat{\mathcal{O}}_{\bar{Y}(\mathfrak{p}), \bar{Q}_{\mathfrak{p}}},
$$

as follows. We first consider the above isomorphism when $\mathfrak{t}=\mathcal{O}_{L}$. Using the description of $\pi^{*}$ on $\bar{Y}_{V}^{\text {ord }}=W_{\emptyset, \mathbb{B}}$ given above, we find that if $\bar{Q}_{\mathfrak{p}} \in W_{\emptyset, \mathbb{B}_{\mathfrak{p}}}(\mathfrak{p})$, then for $\beta \in \mathbb{B}_{\mathfrak{p}}$, we have $\pi^{*}(\mathfrak{p})\left(t_{\beta}\right)=\pi^{*}\left(t_{\beta}\right)=y_{\beta}^{p}=y_{\beta, \mathfrak{p}}^{p}$, where the first and last equalities are via the isomorphism above. On the other hand, considering the above isomorphism when $\mathfrak{t}=(p)$, and using the description of $\pi^{*}$ on $\bar{Y}_{F}^{\text {ord }}=W_{\mathbb{B}, \emptyset}$, we can similarly argue that for $\bar{Q}_{\mathfrak{p}} \in W_{\mathbb{B}_{\mathfrak{p}}, \emptyset}(\mathfrak{p})$, and $\beta \in \mathbb{B}_{\mathfrak{p}}$, we have $\pi^{*}(\mathfrak{p})\left(t_{\beta}\right)=\pi^{*}\left(t_{\beta}\right)=x_{\beta}=x_{\beta, \mathfrak{p}}$. Putting these together, we can calculate the map

$$
\pi^{*}: \widehat{\mathcal{O}}_{\bar{X}, \bar{P}} \rightarrow \widehat{\mathcal{O}}_{\bar{Y}, \bar{Q}}
$$

at a point $\bar{Q} \in W_{\mathbb{B}_{\mathfrak{t}}, \mathbb{B}_{\mathfrak{t}^{*}}}$, as follows. Let $\beta \in \mathbb{B}_{\mathfrak{p}}$. Then, we can write

$$
\pi^{*}\left(t_{\beta}\right)=\pi(\mathfrak{p})^{*}\left(t_{\beta}\right)=\left\{\begin{array}{cc}
x_{\beta, \mathfrak{p}}=x_{\beta} & \bar{Q}_{\mathfrak{p}} \in W_{\mathbb{B}_{\mathfrak{p}}, \emptyset}(\mathfrak{p}) \Leftrightarrow \mathfrak{p} \mid \mathfrak{t}, \\
y_{\beta, \mathfrak{p}}^{p}=y_{\beta}^{p} & \bar{Q}_{\mathfrak{p}} \in W_{\emptyset, \mathbb{B}_{\mathfrak{p}}}(\mathfrak{p}) \Leftrightarrow \mathfrak{p} \mid \mathfrak{t}^{*},
\end{array}\right.
$$

where all the equalities (but the second one) are via the isomorphism above. Renaming the $x_{\beta}, y_{\beta}$ to $z_{\beta}$ as in the second formulation of Theorem 2.4.1, the claim follows.

Define $\mathfrak{Y}_{\text {rig }}(\mathfrak{t})$ to be the Raynaud generic fibre of the completion of $\mathbb{Y}(\mathfrak{t})$ along its special fibre. For $Q \in \mathfrak{Y}_{\text {rig }}(\mathfrak{t})$, and $\beta \in \mathbb{B}_{\mathfrak{t}}$, we define

$$
\nu_{\beta}(Q)= \begin{cases}1 & \beta \in \eta(\bar{Q})-I(\bar{Q}), \\ \nu\left(x_{\beta, \mathfrak{t}}(Q)\right) & \beta \in I(\bar{Q}), \\ 0 & \beta \notin \eta(\bar{Q}),\end{cases}
$$

where $x_{\beta, \mathfrak{t}}$ 's are variables at $\bar{Q}$, a closed point of $\bar{Y}(\mathfrak{t})$, chosen in the same way as in Theorem 2.4.1. It follows that if $Q \in \mathfrak{Y}_{\text {rig }}$, and $Q_{\mathfrak{t}}$ denotes its image in $\mathfrak{Y}_{\text {rig }}(\mathfrak{t})$, then $\nu_{\beta}(Q)=\nu_{\beta}\left(Q_{\mathfrak{t}}\right)$ if 
$\beta \in \mathbb{B}_{\mathfrak{t}}$. We can generalize the definition of $\mathcal{U}, \mathcal{V}, \mathcal{W}, \mathcal{V}_{\mathfrak{p}}, \mathcal{W}_{\mathfrak{p}}$ in 5.3 in the obvious way to obtain admissible opens in $\mathfrak{Y}_{\text {rig }}(\mathfrak{t})$ denoted $\mathcal{V}(\mathfrak{t}), \mathcal{W}(\mathfrak{t}), \mathcal{V}_{\mathfrak{p}}(\mathfrak{t}), \mathcal{W}_{\mathfrak{p}}(\mathfrak{t})$ (for any ideal $\mathfrak{p} \mid \mathfrak{t}$ ). For example,

$$
\mathcal{U}(\mathfrak{t})=\left\{P \in \mathfrak{X}_{\text {rig }}: \nu_{\beta}(P)+p \nu_{\sigma^{-1} \circ \beta}(P)<p \quad \forall \beta \in \mathbb{B}_{\mathfrak{t}}\right\} .
$$

Similarly, we can define

$$
\begin{array}{ll}
\mathfrak{X}_{\text {rig }}^{\circ}(\mathfrak{t})=\left\{P \in \mathfrak{X}_{\text {rig }}(\mathfrak{t}): \nu_{\beta}(P)=0\right. & \left.\forall \beta \in \mathbb{B}_{\mathfrak{t}}\right\}, \\
\mathfrak{Y}_{\text {rig }}^{\circ}(\mathfrak{t})=\left\{Q \in \mathfrak{Y}_{\text {rig }}(\mathfrak{t}): \nu_{\beta}(Q)=0\right. & \left.\forall \beta \in \mathbb{B}_{\mathfrak{t}}\right\} .
\end{array}
$$

As before, we can apply Hensel's lemma to obtain a morphism $s^{\circ}(\mathfrak{t}): \mathfrak{X}_{\text {rig }}^{\circ}(\mathfrak{t}) \rightarrow \mathfrak{Y}_{\text {rig }}^{\circ \circ}(\mathfrak{t})$ which is a section to $\pi(\mathfrak{t})$. Applying the same method as in the proof of Theorem 5.3 .1 , we can prove the following.

Theorem 7.1.3. Let notation be as above.

(1) $\pi(\mathfrak{t})(\mathcal{V}(\mathfrak{t}))=\mathcal{U}(\mathfrak{t})$.

(2) There is a section $s^{\dagger}(\mathfrak{t}): \mathcal{U}(\mathfrak{t}) \rightarrow \mathcal{V}(\mathfrak{t})$ to $\pi(\mathfrak{t})$, extending $s^{\circ}(\mathfrak{t}): \mathfrak{X}_{\text {rig }}^{\circ} \rightarrow \mathfrak{Y}_{\text {rig }}^{\circ}(\mathfrak{t})$.

Let $K \supseteq W(\kappa)$ be a completely valued field. Let $\underline{A} / K$ be an abelian variety corresponding to a point of non-cuspidal reduction $P \in \mathcal{U}(\mathfrak{t})$. Let $Q=s^{\dagger}(\mathfrak{t})(P)$ correspond to $\left(\underline{A}, H_{\mathfrak{t}}\right)$. We call $H_{\mathfrak{t}}$ the $\mathfrak{t}$-canonical subgroup of $\underline{A}$. Again, we can prove:

Theorem 7.1.4. Let $\underline{A} / K$ be an abelian variety corresponding to a point of non-cuspidal reduction $P \in \mathcal{U}(\mathfrak{t})$. Enlarging $K$, we can assume that there exists $r_{\mathfrak{t}}$ in $K$ such that $\operatorname{val}\left(r_{\mathfrak{t}}\right)=$ $\max \left\{\nu_{\beta}(\underline{A}): \beta \in \mathbb{B}_{\mathfrak{t}}\right\}$. The $\mathfrak{t}$-canonical subgroup of $\underline{A}$ reduces to $\operatorname{Ker}(\mathrm{Fr})[\mathfrak{t}]$ modulo $p / r_{\mathfrak{t}}$.

Using the above-explained relationship between the various moduli-spaces we have defined, it is easy to see that if $\underline{A}$ has a $\mathfrak{t}$-canonical subgroup $H_{\mathfrak{t}}$, and if $\mathfrak{z} \mid \mathfrak{t}$, then it also has a $\mathfrak{z}$-canonical subgroup $H_{\mathfrak{z}}$, which satisfies $H_{\mathfrak{z}}=H_{\mathfrak{t}}[\mathfrak{z}]$. In particular, if $\underline{A}$ has a canonical subgroup $H$, then

$$
H=\oplus_{\mathfrak{p} \mid p} H[\mathfrak{p}],
$$

and for each $\mathfrak{p} \mid p$, the subgroup $H[\mathfrak{p}]$ is the $\mathfrak{p}$-canonical subgroup of $\underline{A}$.

Finally, we mention that an analogue of Theorem 5.4.3 holds for a general $\mathfrak{Y}_{\text {rig }}(\mathfrak{t})$, the formulation of which we leave to the reader. Applied to $\mathfrak{t}=\mathfrak{p}$, this completely determines the $p$-adic geometry of the partial $U$-operator, $U_{\mathfrak{p}}$, viewed as a correspondence on $\mathcal{V}(\mathfrak{p}) \cup \mathcal{W}(\mathfrak{p})$, the not-too-singular locus of $\mathfrak{Y}_{\text {rig }}(\mathfrak{p})$.

\section{REFERENCES}

[1] Abbes, A.; Mokrane, A.: Sous-groupes canoniques et cycles évanescents $p$-adiques pour les variétés abéliennes. Publ. Math. Inst. Hautes Études Sci. No. 99 (2004), 117-162.

[2] Andreatta, F.; Gasbarri, C.: The canonical subgroup for families of abelian varieties. Compos. Math. 143 (2007), no. 3, 566-602.

[3] Andreatta, F.; Goren, E. Z.: Geometry of Hilbert modular varieties over totally ramified primes. Int. Math. Res. Not. 2003, no. 33, 1786-1835.

[4] Andreatta, F.; Goren, E. Z.: Hilbert modular forms: $\bmod p$ and $p$-adic aspects. Mem. Amer. Math. Soc. 173 (2005), no. 819 . 
[5] Berthelot, P.: Cohomologie rigide et cohomologie rigide support propre. Premire partie, Prpublication IRMAR 96-03, 89 pages (1996).

[6] Buzzard, K.: Analytic continuation of overconvergent eigenforms. J. Amer. Math. Soc. 16 (2003), 29-55.

[7] Buzzard, K.; Taylor, R.: Companion forms and weight one forms. Ann. of Math. (2) 149 (1999), no. 3, 905-919.

[8] Chai, C.-L. Arithmetic minimal compactification of the Hilbert-Blumenthal moduli spaces. Ann. of Math. (2) 131 (1990), no. 3, 541-554.

[9] Yu, C.-F.:

[10] Coleman, R. F.: Classical and overconvergent modular forms. Invent. Math. 124 (1996), no. 1-3, $215-241$.

[11] Conrad, B.: Higher-level canonical subgroups in abelian varieties. Preprint.

[12] Deligne, P.; Ribet, K. A.: Values of abelian $L$-functions at negative integers over totally real fields. Invent. Math. 59 (1980), no. 3, 227-286.

[13] de Jong, A. J.: The moduli spaces of principally polarized abelian varieties with $\Gamma_{0}(p)$-level structure. J. Algebraic Geom. 2 (1993), no. 4, 667-688.

[14] Deligne, P.; Pappas, G.: Singularités des espaces de modules de Hilbert, en les caractéristiques divisant le discriminant. Compositio Math. 90 (1994), no. 1, 59-79.

[15] Eisenbud, D.: Commutative algebra. With a view toward algebraic geometry. Graduate Texts in Mathematics, 150. Springer-Verlag, New York, 1995.

[16] Fargues, L.: Application de Hodge-Tate duale d'un groupe de Lubin-Tate, immeuble de Bruhat-Tits du groupe linéaire et filtrations de ramification. Duke Math. J. 140 (2007), no. 3, 499-590.

[17] Freitag, E.: Hilbert modular forms. Springer-Verlag, Berlin-Heidelberg,1990.

[18] Goren, E. Z.: Hasse invariants for Hilbert modular varieties. Israel J. Math. 122 (2001), 157-174.

[19] Goren, Eyal Z. Lectures on Hilbert modular varieties and modular forms. With the assistance of Marc-Hubert Nicole. CRM Monograph Series, 14. American Mathematical Society, Providence, RI, 2002.

[20] Goren, E. Z.; Kassaei, P. L.: The canonical subgroup: a "subgroup-free" approach. Comment. Math. Helv. 81 (2006), no. 3, 617-641.

[21] Goren, E. Z.; Oort, F.: Stratifications of Hilbert modular varieties. J. Algebraic Geom. 9 (2000), no. 1, $111-154$

[22] Grothendieck, A.: Éléments de géométrie algébrique. III. Étude cohomologique des faisceaux cohérents. Inst. Hautes Études Sci. Publ.Math. (1961), no. 11.

[23] Grothendieck, A.: Groupes de Barsotti-Tate et cristaux de Dieudonné. Séminaire de Mathématiques Supérieures, No. 45 (1970). Les Presses de l'Université de Montréal, Montreal, Que., 1974.

[24] Hartshorne, R.: Algebraic geometry. Graduate Texts in Mathematics, No. 52. Springer-Verlag, New YorkHeidelberg, 1977.

[25] Kassaei, P. L.: $\mathscr{P}$-adic modular forms over Shimura curves over totally real fields. Compos. Math. 140 (2004), no. 2, 359-395.

[26] Kassaei, P. L.: A gluing lemma and overconvergent modular forms. Duke Math. J. 132 (2006), no. 3, 509-529.

[27] Kassaei, P. L: Overconvergence, analytic continuation, and classicality: the case of curves. To appear in Journal für die reine und angewandte Mathematik .

[28] Katz, N. M.: p-adic properties of modular schemes and modular forms. Modular functions of one variable, III, pp. 69-190. Lecture Notes in Mathematics, Vol. 350, 1973.

[29] Kisin, M.; Lai, K. F.: Overconvergent Hilbert modular forms. Amer. J. Math. 127 (2005), no. 4, $735-783$.

[30] Oda, T.: The first de Rham cohomology group and Dieudonné modules. Ann. Sci. École Norm. Sup. (4) 2, 1969, 63-135.

[31] Pappas, G.: Arithmetic models for Hilbert modular varieties. Compositio Math. 98 (1995), no. 1, 43-76.

[32] Rapoport, M.: Compactifications de l'espace de modules de Hilbert-Blumenthal. Compositio Math. 36 (1978), no. 3, 255-335.

[33] Serre, J.-P.: Formes modulaires et fonctions zêta $p$-adiques. Modular functions of one variable, III (Proc. Internat. Summer School, Univ. Antwerp, 1972), pp. 191-268. Lecture Notes in Math., Vol. 350, Springer, Berlin, 1973.

[34] Stamm, H.: On the reduction of the Hilbert-Blumenthal-moduli scheme with $\Gamma_{0}(p)$-level structure. Forum Math. 9 (1997), no. 4, 405-455.

[35] Tian, Y.: Canonical subgroups of Barsotti-Tate groups. To appear in Ann. of Math.

Department of Mathematics and Statistics, McGill University, 805 Sherbrooke St. W., Montreal H3A 2K6, QC, CANADA. 
Department of Mathematics, King's College London, Strand, London WC2R 2LS, United KingDOM.

E-mail address: eyal.goren@mcgill.ca; kassaei@alum.mit.edu 